NASA Contractor Report 198162

ICASE Report No. 95-39
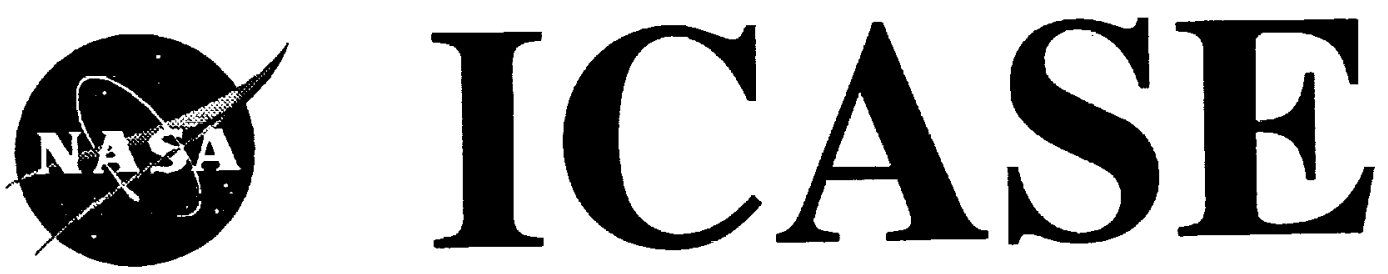

\title{
ACOUSTIC FIELD IN UNSTEADY MOVING MEDIA
}

F. Bauer

L. Maestrello

L. Ting
(NASA-CR-198162) ACDUSTIC FIELD IN UNSTEADY MOVING MEDIA Final Report (ICASE) $48 \mathrm{P}$
N95-27179

Unclas

Contract No. NAS1-19480

May 1995

Institute for Computer Applications in Science and Engineering

NASA Langley Research Center

Hampton, VA 23681-0001 
$=-\infty$ 


\title{
ACOUSTIC FIELD IN UNSTEADY MOVING MEDIA
}

\author{
F. Bauer \\ Courant Institute of Mathematical Sciences \\ New York University \\ New York, NY 10012 \\ L. Maestrello \\ NASA Langley Research Center \\ Hampton, VA 23681-0001 \\ L. Ting \\ Courant Institute of Mathematical Sciences \\ New York University \\ New York, NY 10012
}

\begin{abstract}
In the interaction of an acoustic field with a moving airframe we encounter a canonical initial value problem for an acoustic field induced by an unsteady source distribution, $q(t, \mathbf{x})$ with $q \equiv 0$ for $t \leq 0$, in a medium moving with a uniform unsteady velocity $U(t) \hat{\imath}$ in the coordinate system $\mathbf{x}$ fixed on the airframe. Signals issued from a source point $S$ in the domain of dependence $\mathcal{D}$ of an observation point $P$ at time $t$ will arrive at point $P$ more than once corresponding to different retarded times, $\tau$ in the interval $[0, t]$. The number of arrivals is called the multiplicity of the point $S$. The multiplicity equals 1 if the velocity $U$ remains subsonic and can be greater when $U$ becomes supersonic. For an unsteady uniform flow $U(t) \hat{\imath}$, rules are formulated for defining the smallest number of $I$ subdomains $V_{i}$ of $\mathcal{D}$ with the union of $V_{i}$ equal to $\mathcal{D}$. Each subdomain has multiplicity 1 and a formula for the corresponding retarded time. The number of subdomains $V_{i}$ with nonempty intersection is the multiplicity $m$ of the intersection. The multiplicity is at most $I$. Examples demonstrating these rules are presented for media at accelerating and/or decelerating supersonic speed.
\end{abstract}

\footnotetext{
*This research was partially supported by the National Aeronautics and Space Administration under NASA Contract No. NAS1-19480 while the third author was in residence at the Institute for Computer Applications in Science and Engineering (ICASE), NASA Langley Research Center, Hampton, VA 236810001. This research was also partially supported by AFOSR Grant F49620-93-0027.

$\dagger$ This is an updated version of NYU CIMS Report BMT94 with additional examples.
} 



\section{Introduction}

In this paper we study the propagation of acoustic waves induced by a spatial source distribution $q(t, \mathbf{x})$ in a uniform medium moving with a time dependent velocity $U(t) \hat{\imath}$ relative to the coordinate system $\mathbf{x}=(x, y, z)$. This study is an outgrowth of recent experimental and theoretical investigations of a model acoustic-panel interaction problem. See for example [1] and [2]. The acoustic source distribution could simulate the noise from jet exhausts, turbulent boundary layers and/or the airframe panel oscillation. For the analysis of the panel oscillation it is convenient to work in the coordinate system $\mathbf{x}=(x, y, z)$, stationary with the airframe, while the flow field is moving with the uniform unsteady velocity $U(t) \hat{\imath}$. In this system, the acoustic potential $\Phi(t, x, y, z)$ is governed by the convective wave equation with variable coefficients, the velocity $U(t)$ and the acceleration $\dot{U}(t)$,

$$
\left[\partial_{t t}^{2}+\dot{U} \partial_{x}+2 U \partial_{t x}^{2}+U^{2} \partial_{x x}^{2}-C^{2} \Delta\right] \Phi(t, x, y, z)=q(t, x, y, z),
$$

where $\Delta$ denotes the Laplacian in $(x, y, z)$ and $C$ stands for the speed of sound of the medium at the ambient condition. For an initial value problem, we impose

$$
\Phi=0, \quad \partial_{t} \Phi=0, \quad \text { at } t=0,
$$

or

$$
q=\Phi=\Phi_{t}=0, \text { for } t \leq 0 .
$$

In general, the solution $\Phi(t, \mathbf{x})$ is constructed indirectly via the solution of the corresponding acoustic field $\bar{\Phi}$ in the coordinate system $\overline{\mathbf{x}}$ with the medium at rest [3]. These two coordinate systems, which coincide at $t=0$, are related by translation, $\mathbf{x}=\overline{\mathbf{x}}+\hat{\imath} X(t)$, i. e.,

$$
\bar{x}=x-X(t), \quad \bar{y}=y, \quad \bar{z}=z, \quad \text { with } \quad X(t)=\int_{0}^{t} U\left(t^{\prime}\right) d t^{\prime} .
$$

From hereon we omit the bars over $y$ and $z$ for the coordinates of $\mathbf{x}$. Figure 1 shows the coordinate $\bar{x}$ with the medium at rest moving with velocity $U(t) \hat{\imath}$ relative to the coordinate $x$. We use $\bar{f}$ and $f$ to denote the same quantity as functions of $\bar{t}, \bar{x}, y, z$ and $t, x, y, z$ respectively with $\bar{t}=t$. Thus we have

$$
\bar{f}(\bar{t}, \bar{x}, y, z)=f(t, x, y, z) \quad \text { with } \quad \partial_{\bar{t}} \bar{f}=\left(\partial_{t}+U \partial_{x}\right) f \text { and } \bar{\nabla} \bar{f}=\nabla f,
$$

where $\bar{\nabla}$ and $\nabla$ denote the gradient operators in $\overline{\mathbf{x}}$ and $\mathbf{x}$. In particular, if $f$ denotes the unsteady source distribution $q$ in the coordinate system, $\mathbf{x}$, the corresponding distribution $\bar{q}$ in $\overline{\mathbf{X}}$ is given by (1.5). In the barred system, the acoustic potential $\bar{\Phi}$ is governed by the simple wave equation,

$$
\left[C^{-2} \partial_{\bar{t} \bar{t}}^{2}-\bar{\Delta}\right] \bar{\Phi}(\bar{t}, \bar{x}, y, z)=\bar{q}(\bar{t}, \bar{x}, y, z)
$$

where $\bar{\Delta}$ denotes the Laplacian in $(\bar{x}, y, z)$. With homogeneous initial conditions (1.2) applied to the barred variables, the potential at point $\bar{P}(\overline{\mathbf{x}})$ at instant $t$, is given by the explicit formula [3],

$$
\bar{\Phi}(t, \bar{x}, y, z)=-\frac{1}{4 \pi} \iiint_{\bar{V}} d \bar{\xi} d \eta d \zeta \frac{\bar{q}\left(\tau, \bar{x}^{\prime}, y^{\prime}, z^{\prime}\right)}{R},
$$

where $\bar{\xi}, \eta, \zeta$ denote the coordinates of a source point $\bar{S}\left(\overline{\mathbf{x}}^{\prime}\right)$ relative to point $\bar{P}(\overline{\mathbf{x}})$, 


$$
\bar{x}^{\prime}=\bar{x}+\bar{\xi}, \quad y^{\prime}=y+\eta, \quad z^{\prime}=z+\zeta,
$$

$R$ denotes the distance from $S$ to $P$ and $\tau$ the retarded time,

$$
R=\left|\overline{\mathbf{x}}^{\prime}-\overline{\mathbf{x}}\right|=\sqrt{\bar{\xi}^{2}+\sigma^{2}} \text { and } \tau=t-R / C \text { where } \sigma=\sqrt{\eta^{2}+\zeta^{2}} .
$$

We now identify the domain of integration $\bar{V}$ as the domain of dependence $\overline{\mathcal{D}}$ of point $\bar{P}$ at time $t$. In the coordinate system, $\bar{\xi}, \eta, \zeta$, the point $\bar{P}$ is the origin of the system and the domain of dependence $\overline{\mathcal{D}}$ is a spherical ball of radius $C t$ centered at the origin, $\bar{P}$, i. e.,

$$
\overline{\mathcal{D}}=\{(\bar{\xi}, \eta, \zeta) \mid 0 \leq R \leq C t\}
$$

It is covered by a family of concentric spheres of radii $R \in[0, C t]$. The signal from a point $\bar{S}$ in $\overline{\mathcal{D}}$ on a sphere of radius $R$ arrives at the origin $\bar{P}$ along the radial line $\bar{S} \bar{P}$ at time $t$ when the signal was initiated at the retarded time $\tau=t-R / C$. Since the domain of dependence is axi-symmetric with respect to the $\bar{\xi}$ axis, we show in Fig. 2 the cross section of the domain $\overline{\mathcal{D}}$, its boundary $\overline{\mathcal{B}}$ and the family of concentric spheres in a meridian plane, the $\bar{\xi} \sigma$ plane. We define the multiplicity of a point by the number of retarded times the signal from the point reaches point $\bar{P}$ at time $t$. Thus the multiplicity of a point in $\bar{D}$ or the multiplicity of $\overline{\mathcal{D}}$, in the barred coordinate system with the medium at rest, is one and hence the domain of integration $\bar{V}$ for the integral on the right hand side of (1.7) is equal to the domain of dependence $\overline{\mathcal{D}}$. Note that the above description of the domain of dependence (1.10) in the barred coordinate system is independent of the velocity of the medium relative to the coordinate system $\mathbf{x}$.

To identify the source distribution $\bar{q}$ at point $\bar{S}\left(\bar{x}^{\prime}, y^{\prime}, z^{\prime}\right)$ at a retarded time $\tau$ with the distribution $q$ at the corresponding point $S\left(x^{\prime}, y^{\prime}, z^{\prime}\right)$ in the moving frame, we relate the coordinate $\bar{x}^{\prime}$ to $x^{\prime}$ by (1.4) at time $\tau$, i. e.,

$$
x^{\prime}=\bar{x}^{\prime}+X(\tau) \text { and } \bar{q}\left(\tau, \bar{x}^{\prime}, y^{\prime}, z^{\prime}\right)=q\left(\tau, x^{\prime}, y^{\prime}, z^{\prime}\right) .
$$

By using (1.4) and (1.5), the left hand side of (1.7) is identified as $\Phi(t, x, y, z)$ and (1.7) becomes an implicit formula for $\Phi$ at point $P(x, y, z)$. Since the dependency on the velocity $U(t) \hat{\imath}$ of the medium appears only via the transformation (1.11) for the source distribution, the solution (1.7) of the initial value problem, (1.6) and (1.2), is valid for all Mach numbers including the transonic range.

To express the potential $\Phi$ as an integral in the coordinate system $\mathbf{x}$, we change the integration variables $\bar{\xi}, \eta, \zeta$ in (1.7) to $\xi, \eta, \zeta$ which are the coordinates of point $S$ relative to $P$. Using (1.4), (1.8) and (1.11), we have $\xi$ as function of $\bar{\xi}$ and $\sigma$ or $\xi$ and $\tau$,

$$
\xi=x^{\prime}-x=\bar{\xi}+X(\tau)-X(t)=\bar{\xi}-\int_{\tau}^{t} U\left(t^{\prime}\right) d t^{\prime},
$$

where $\tau=t-R / C, R=\sqrt{\bar{\xi}^{2}+\sigma^{2}}$ and $\sigma=\sqrt{\eta^{2}+\zeta^{2}}$.

The Jacobian determinant of the coordinate transformation is

$$
J=\frac{\partial(\xi, \eta, \zeta)}{\partial(\bar{\xi}, \eta, \zeta)}=\frac{\partial \xi}{\partial \bar{\xi}}=\frac{R-M \bar{\xi}}{R}=1-M \cos \psi
$$


Here $R, \theta, \psi$ with $0 \leq \psi \leq \pi$ represent the spherical coordinates while $\bar{\xi}, \sigma, \theta$ the cylindrical coordinates with $\eta=\sigma \cos \theta$ and $\zeta=\sigma \sin \theta$. Note that in the transformation (1.12), $\eta$ and $\zeta$ and hence $\sigma$ and $\theta$ remain the same. The cylindrical coordinates in the $\xi, \eta, \zeta$ space are $\xi, \sigma$ and $\theta$.

The spheres $\bar{S}$ of constant $\tau$ in a subsonic interval, where $M(\tau)<1$, cover a subsonic region in $\overline{\mathcal{D}}$. The spheres in a supersonic interval, where $M(\tau)>1$, cover a supersonic region. In a subsonic region, the Jacobian remains positive. In a supersonic region, there is a critical surface, $\overline{\mathcal{J}}$, on which the radial velocity component is sonic,

$$
J=1-M(\tau) \cos \psi=0 \quad \text { or } \quad \sigma=\bar{\xi} \sqrt{M^{2}-1} \geq 0 .
$$

The transformation (1.12) maps the domain of dependence $\overline{\mathcal{D}}$ of the origin $\bar{P}$ of the $\bar{\xi}, \eta, \zeta$ space to the domain of dependence $\mathcal{D}$ of the origin $P$ of the $\xi, \eta, \zeta$ space. The critical surface $\overline{\mathcal{J}}$ in a supersonic region is then mapped to surface $\mathcal{J}$. Since the Jacobian $J$ changes sign across $\overline{\mathcal{J}}$, the neighborhoods on the two sides of $\overline{\mathcal{J}}$ are mapped to the same side of $\mathcal{J}$. Thus the inverse transformation of (1.12) is not single valued and hence the multiplicity of a point in $\mathcal{D}$ can be greater than 1 . For a given point $(\xi, \sigma)$ and time $t,(1.12)$ becomes an implicit equation for $\bar{\xi}$ because the lower limit of the integral $\tau=t-R / C$ and $R$ are related to $\bar{\xi}$ by (1.9). Using (1.9) and (1.12), we get an implicit equation for $R(\tau, \xi, \sigma)$,

$$
\left.R^{2}=\left[\xi+\int_{\tau}^{t} U\left(t^{\prime}\right) d t^{\prime}\right)\right]^{2}+\sigma^{2}
$$

The root(s) of (1.15) in $[0, C t]$ depend on the unsteady velocity $U\left(t^{\prime}\right)$.

Using (1.11) to (1.13), we convert (1.7) to a volume integral in the $\xi, \eta, \zeta$ space,

$$
\begin{aligned}
\Phi(t, x, y, z) & =-\frac{1}{4 \pi} \iiint_{\mathcal{D}} d \xi d \eta d \zeta \frac{q(\tau, x+\xi, y+\eta, z+\zeta)}{|R-M \bar{\xi}|} \\
& =-\frac{1}{4 \pi} \sum_{i=1}^{I} \iiint_{V_{i}} d \xi d \eta d \zeta \frac{q\left(\tau_{i}, x+\xi, y+\eta, z+\zeta\right)}{\left|R_{i}-M_{i} \bar{\xi}_{i}\right|} .
\end{aligned}
$$

To evaluate the integral over the domain of dependence $\mathcal{D}$ in (1.16a) we need to relate $R$ and $\bar{\xi}$ to $\xi, \sigma$ and $t$, that is, to find all the roots, $R_{i}$ of $(1.15)$ in $[0, C t]$ or the retarded time $\tau_{i}$ in $[0, t]$ and identify the domain $V_{i}$ in the $\xi, \eta, \zeta$ space for each $R_{i}$. Then $\Phi$ is written as the sum of integrals over the smallest number $I$ of domains $V_{i}$ in (1.16b). It is the purpose of this paper to formulate the rules identifying these domains $V_{i}$ for a medium moving at an unsteady speed, $U(t)$. First, we review briefly the solutions given in [2] and [3] for the following three special cases; 1) moving at an unsteady subsonic speed, 2) moving at a constant supersonic speed, and 3) moving with constant acceleration.

1.1, Unsteady Subsonic Speed For this case, we have $M(\tau)<1, \tau \in[0, t]$, the forward and backward speeds of propagation $C \pm U$ are positive and hence the family of concentric spheres covering $\bar{V}$ is mapped to a family of nested spheres covering the spherical ball $\mathcal{D}$ bounded by $\mathcal{S}_{0}$. Hence $I=1$ and the domain of dependence $\mathcal{D}$ has multiplicity 1 . This 
result reduces to the well known result for a steady subsonic flow, at a constant $M<1$, for which (1.15) has a unique and explicit solution for $\xi, \eta, \zeta$ in $\mathcal{D}$ and the denominator in (1.16) becomes the pseudo-distance [3],

$$
R-M \xi=R^{*}=\sqrt{\xi^{2}+\left(1-M^{2}\right) \sigma^{2}} .
$$

The result that the domain of integration in (1.16) is equal to $\mathcal{D}$ remains valid even when there is a sonic interval, where $M(\tau)=1$ for $0 \leq t_{1} \leq \tau \leq t_{2} \leq t$, during which, the nested spheres will be in contact at one point on the $\xi$-axis with $\xi=C(t-\tau)-X(t)+X(\tau)=$ $C\left(t-t_{1}\right)-X(t)+X\left(t_{1}\right)$. Although the multiplicity at the point of contact is infinite, the integral in (1.16) remains finite because the acoustic field is initiated at $t=0$.

1.2, Constant Supersonic Speed For the case of a medium moving at constant supersonic speed, $M>1$, the solution is well known [3]. The domain of dependence $\mathcal{D}$ of point $P$ lies in the backward Mach cone from $P$. The cone is

$$
\xi=-\sqrt{\left(M^{2}-1\right) \sigma^{2}} .
$$

To evaluate the integral we need to relate $R$ and $\bar{\xi}$ to $\xi, \sigma$ and $t$, The domain $\mathcal{D}$ is covered by a one parameter family of spheres of radius $R=C(t-\tau)$ centered on the $\xi$-axis at $\xi=-M R$ for $\tau \in[0, t]$. The Mach cone is the envelope of these spheres. The circle of tangency of a sphere with the cone divides the sphere into a large and a small surface, convex and concave away from the cone respectively. Fig. 3 shows the cross section of the Mach cone and the spheres in the meridian plane, the $\xi \sigma$ plane. As an initial value problem, we have $\tau \geq 0$ or $R \leq C t$, thus the domain of dependence $\mathcal{D}$ is bounded by the backward Mach cone (1.18) and the convex part of the spherical surface $S_{0}$ with radius $C t$ centered at $\xi=-U t$ and azimuthal angle $\psi \in[\pi / 2-\alpha, \pi]$, where $\alpha$ is the Mach angle. Note that the mirror image of the domain of dependence $\mathcal{D}$ with respect to the plane $\xi=0$ is the domain of influence of a source $S$ at the origin for the duration $[0, t]$ described in [3]. With a constant $M>1,(1.15)$ reduces to a quadratic equation for $R$ and the two roots are [3],

$$
R^{ \pm}=-\left[M \xi \pm R^{*}\right] /\left(M^{2}-1\right),
$$

where $R^{*}=\sqrt{\xi^{2}-\left(M^{2}-1\right) \sigma^{2}}$. The denominator of the integrand in (1.16) becomes $R^{*}$ and the integral can be written as the sum of two integrals over the domains $V^{-}$and $V^{+}$. Equation (1.16) becomes,

$$
\Phi(t, x, y, z)=-\frac{1}{4 \pi}\left[\iiint_{V^{+}}+\iiint_{V^{-}}\right] \frac{q(\tau, x+\xi, y+\eta, z+\zeta)}{R^{*}} d \xi d \eta d \zeta,
$$

with $\tau=t-R^{ \pm}$in $V^{ \pm}$and $I=2$. The domain of integration $V^{+}$is bounded by the Mach cone and convex part of the sphere $S_{0}$ and is equal to $\mathcal{D}$. The domain of integration $V^{-}$ is bounded by the concave part of the sphere $S_{0}$ with $\phi \in[0, \alpha]$. Note that the superscript of $V$ gives the sign of $J$ in $V^{ \pm}$. Since $V^{-} \in V^{+}=\mathcal{D}$, the multiplicity of $\mathcal{D} \cap V^{-}$is two while the multiplicity of the complement, the spherical ball inside $S_{0}$, is one. The meaning of $R^{ \pm}$and the propagation of signals from point $S$ at $\tau^{ \pm}$to $P$ at time $t$ are described in Appendix A. 
Without making use of the explicit solution (1.19) of (1.15), the results in [3] quoted above were obtained by a different method in [2]. It begins with the partition of the domain of dependence $\overline{\mathcal{D}}$, the spherical ball (1.10), into two nonoverlapping subdomains, $\bar{V}^{ \pm}$, by the critical surface $\overline{\mathcal{J}},(1.14)$, which is the circular cone, $\psi=\arccos (1 / M)=\pi / 2-\alpha$. Shown in Fig. 4 is the partition of $\overline{\mathcal{D}}$ in a meridian $\bar{\xi} \sigma$ plane by the critical curve $\overline{\mathcal{J}}$, the radial line $\bar{P} \bar{T}$, into two subdomains $\bar{V}^{-}$and $\bar{V}^{+}$, with $0 \leq \psi<\pi / 2-\alpha$ and $\pi / 2-\alpha<\psi \leq \pi$ respectively. The image of $\overline{\mathcal{J}}$ in the $\xi \sigma$ plane in Fig. 3 is the backward Mach line $P T$ and the images of $\bar{V}^{ \pm}$are the domains of integration $V^{ \pm}$described in the preceding paragraph.. The procedure for the identification of the domains of integration in the $\xi, \eta, \zeta$ space, introduced in [2], demonstrates the important role played by the critical surface, which does not exist in an unsteady subsonic region. Since this procedure does not need the explicit solution of (1.15) or (1.12), it is suitable for generalization to an unsteady speed $U(\tau)$ with several supersonic intervals. As an illustration or rather a clue to the generalization, we mention the special case of constant accelerating motion considered in [2].

1.3 Constant Accelerating Motion The interaction of an acoustic field with a baffled panel in the horizontal $x z$ plane was studied in [2], for the panel moving with constant acceleration to a supersonic speed, i. e., $U=C \tau$. With $M=\tau$ for $\tau \in[0, t]$ and $t>1$, there is a supersonic interval $1<\tau \leq t$. This special case shows that in addition to the partition of $\overline{\mathcal{D}}$ by the critical surface $\overline{\mathcal{J}}$ in a supersonic region, further partitions may be needed, related to the geometrical properties of $\overline{\mathcal{J}}$ and its image $\mathcal{J}$. See Figs. 4 and 5 . This case is discussed in $\S 4$ as one of the examples for the application of the rules of partition formulated in $\S 3$.

In the next section, we study the geometry of the critical surface $\overline{\mathcal{J}}$ and the corresponding singular points on the image $\mathcal{J}$. We then use these studies to formulate in $\S 3$ the rules for the partition of the domain of dependence, $\overline{\mathcal{D}}$, into nonoverlapping subdomains $\bar{V}_{j}$ 's for an unsteady velocity, $U(\tau), \tau \in[0, t]$. and show that the partition has the required property that the transformation (1.12) from a $\bar{V}_{j}$ to its image $V_{j}$ in the $\xi, \eta, \zeta$ space is one to one. In particular, the value of $R$ or $\tau$ defined by (1.15) for a point in $V_{j}$ is uniquely defined. Thus the domain of integration $\mathcal{D}$ in (1.16) is the sum of those images $V_{j}$ 's. Since those images may overlap each other in the $\xi, \eta, \zeta$ space, signals from a point $S$ in the space lying in $m$ of those images will reach point $P m$-times. Hence $m$ is the multiplicity of point $S$. The rules for counting the multiplicity of a point in $D$ are also stated in $\S 3$. The analyses in $\$ 2$ and $\mathbf{3}$ justify the assertions made in [2] for the case of a constantly accelerating speed. Additional examples with unsteady velocities, accelerating and/or decelerating at different ranges of Mach numbers are presented in $\S 4$ to demonstrate the application of the rules of partition of $\overline{\mathcal{D}}$ and to show the singularities of $\mathcal{J}$ in the $\xi, \eta, \zeta$ space.

\section{The Geometry of the Critical Surface and its Image}

In $\S 1$, the domain of dependence $\overline{\mathcal{D}}$ of point $\bar{P}$ at time $t$ and the critical surface $\overline{\mathcal{J}}$ in the coordinate system $(\bar{\xi}, \eta, \zeta)$ with the medium at rest are defined by (1.10) and (1.14). The transformation from the system $(\bar{\xi}, \eta, \zeta)$ to $(\xi, \eta, \zeta)$ defined by (1.12) at time $\tau$ is a pure translation of $(X(\tau)-X(t))$ along the $\bar{\xi}$ axis. We reemphasize the following points $\dagger$

$\dagger$ Numbers in curly brackets refer to statement numbers in $\S 2$. 
\{1\} The domain $\overline{\mathcal{D}}$ is a spherical ball of radius $C t$ centered at point $\bar{P}$ and for each point on a concentric sphere of radius $R \in[0, C t]$, the retarded time is $t-R / C$. Thus $\overline{\mathcal{D}}$ has multiplicity one, independent of the source distribution and the velocity $U(\tau) \hat{\imath}$ of the medium.

$\{2\}$ The critical surface $\overline{\mathcal{J}}$, on which the Jacobian determinant $J$ vanishes, appears in $\overline{\mathcal{D}}$ only in a supersonic region. The surface is axisymmetric with respect to the $\bar{\xi}$-axis, is independent of the source distribution but depends on the velocity $U(\tau)$ of the medium. See for example Figs. 2 and 4

\{3\} The transformation (1.12) relates the axial variable $\bar{\xi}$ to $\xi$ while the other two variables $\eta$ and $\zeta$ or $\sigma$ and $\theta$ remain unchanged. The transformation is also independent of the source distribution but depends on $U(\tau)$. Thus the images of the spherical ball $\overline{\mathcal{D}}$, the concentric spheres of constant $\tau$ and the critical surface $\overline{\mathcal{J}}$ in the $\xi, \eta, \zeta$ space are axisymmetric with respect to the $\xi$-axis.

\{4\} A spherical cap of radius $R$ centered at the origin of the $\bar{\xi}, \eta, \zeta$ space is mapped to the same spherical cap centered at $\hat{\imath}(X(\tau)-X(t))$ in the $\xi, \eta, \zeta$ space with the same retarded time $\tau=t-R / C$. See for example Figs. 3 and 5 .

Because of the axisymmetry, it suffices to study the geometry of the surfaces $\overline{\mathcal{J}}$ and its image $\mathcal{J}$ in a meridian plane, the $\bar{\xi} \sigma$ plane, and the corresponding $\xi \sigma$ plane. We retain the same symbols $\overline{\mathcal{D}}, \overline{\mathcal{J}}, \bar{V}_{j}$ for their cross sections in a meridian plane and the same unbarred symbols for the images. The geometrical properties of the critical curve $\overline{\mathcal{J}}$ and its image $\mathcal{J}$ will be described in $\S \mathbf{2 . 1}$ and $\S \mathbf{2 . 2}$ respectively and be employed in $\S 3$ to formulate the rules for the partition of $\overline{\mathcal{D}}$ into nonoverlapping subdomains $\bar{V}_{j}, j=1,2, \cdots$ such that the mapping of a $\bar{V}_{j}$ to its image $V_{j}$ is one to one.

\subsection{The critical curve $\overline{\mathcal{J}}$ in a meridian plane, the $\bar{\xi} \sigma$ plane}

In the meridian plane, the two spherical coordinates $R$ and $\psi$ become the polar coordinates, and the domain of dependence $\overline{\mathcal{D}}$ in the plane is a semicircular disc with $0 \leq R \leq C t$ and $0 \leq \psi \leq \pi$. Let us consider the signals initiated in a supersonic interval, $i$. e., $M(\tau)>1, \quad \tau \in\left[t_{*}, t^{*}\right]$, and $M=1$ at $t_{*}$ if $t_{*}>0$ and at $t^{*}$ if $t^{*}<t$. The signals come from points in the supersonic ring $C\left(t-t^{*}\right) \leq R \leq C\left(t-t_{*}\right)$ in $\overline{\mathcal{D}}$. The critical curve $\overline{\mathcal{J}}$ in the polar coordinates is defined by,

$$
\cos \psi=1 / M(\tau) \text { and } R=C(t-\tau),
$$

and in the rectangular coordinates $(\bar{\xi}, \sigma)$ by

$$
\bar{\xi}=R \cos \psi=C(t-\tau) / M \quad \text { and } \quad \sigma=R \sin \psi=C(t-\tau)\left[1-M^{-2}\right]^{1 / 2},
$$

with $\tau \in\left[t_{*}, t^{*}\right]$. See for example Fig. 4 , where $t_{*}=1$ and $t^{*}=t$. Since the Mach angle $\alpha(\tau)=\pi / 2-\psi$ we have from $(2.1)$,

\{5\} The radial line to point $\bar{Q}(R, \psi)$ on $\overline{\mathcal{J}}$ is orthogonal to the Mach line at $\bar{Q}$. The polar angle $\psi$ of $\bar{Q}$ reaches a local maximum (minimum) when $M$ or $U$ reaches a local maximum (minimum). 
The rates of change of $\sigma$ and $\bar{\xi}$ are

$$
\sigma^{\prime}(\tau)=\frac{R M^{\prime}-C M\left(M^{2}-1\right)}{M^{2} \sqrt{M^{2}-1}}, \quad \bar{\xi}^{\prime}(\tau)=\frac{-C M-R M^{\prime}}{M^{2}}
$$

and the slope of $\overline{\mathcal{J}}$ is

$$
\frac{d \sigma}{d \bar{\xi}}=\frac{\left[R M^{\prime}-C M\left(M^{2}-1\right)\right]}{-\left(C M+R M^{\prime}\right) \sqrt{M^{2}-1}} .
$$

From hereon we use the prime to denote the $\tau$-derivative. From (2.3), we see that $\sigma^{\prime}(\tau)<0$ when $M^{\prime} \leq 0$, therefore, $\sigma^{\prime}$ and the slope vanish only when $M^{\prime}>0$ while $\bar{\xi}^{\prime}(\tau)<0$. Thus $\{6\}$ The ordinate $\sigma$ of $\overline{\mathcal{J}}$ attains an extremum only in an accelerating subregion, while $\bar{\xi}$ decreases.

Since $\sigma$ remains unchanged in the transformation from the $\bar{\xi} \sigma$ plane to $\xi \sigma$ plane, the mapping of a neighborhood of the curve $\overline{\mathcal{J}}$ to its image is defined by the mapping of a horizontal line segment at constant $\sigma$. A small segment which crosses over $\overline{\mathcal{J}}$ at a point with nonzero slope, or $\sigma^{\prime} \neq 0$, will cross over $\overline{\mathcal{J}}$ only once.

Now consider an extremum point, say $\bar{K}_{e}$, i. e., $\sigma_{e}^{\prime}=0$ at $\tau_{e}$, we have

$$
\bar{\xi}_{e}^{\prime}=-C M_{e} \quad \text { and } \quad \sigma_{e}^{\prime \prime}=\frac{R_{e} M_{e}^{\prime \prime}-3 C M_{e}^{2} M_{e}^{\prime}}{M_{e}^{2} \sqrt{M_{e}^{2}-1}}
$$

We have assumed that $U^{\prime \prime}(\tau)$ or $M^{\prime \prime}$ exists, therefore, $\sigma_{e}^{\prime \prime}$ exists with $M_{e}>1$. This is a realistic assumption and will simplify our discussion that follows but is not essential.

When the extremum point is neither a maximum nor a minimum, $\sigma^{\prime}$ does not change sign as $\tau$ crosses $\tau_{e}$ and $\sigma_{e}^{\prime \prime}=0$. A horizontal segment close to point $\bar{K}_{e}$ will intersect $\overline{\mathcal{J}}$ only once.

When $\sigma^{\prime \prime} \neq 0$ at a critical point say $\bar{K}_{1}$, it is either a local maximum or minimum point. The curve $\overline{\mathcal{J}}$ near $\bar{K}_{1}$ behaves as a parabola with $\left(\sigma-\sigma_{1}\right) \sigma_{1}^{\prime \prime}>0$. For a given $\sigma$, there are two roots,

$$
\bar{\xi}-\bar{\xi}_{1}= \pm C M_{1}\left[\frac{2\left(\sigma-\sigma_{1}\right)}{\sigma_{1}^{\prime \prime}}\right]^{1 / 2}+O\left(\sigma-\sigma_{1}\right)
$$

Thus a horizontal segment near point $\bar{K}_{1}$ crosses over $\overline{\mathcal{J}}$ twice if it is below (above) $\bar{K}_{1}$ where $\sigma$ is a local maximum (minimum). This point will be elaborated in $\{11 a\}$ and $\{11 b\}$. We need to locate the local maximum and minimum points on $\overline{\mathcal{J}}$. Let the $n$-th point and the corresponding time be denoted by $\bar{K}_{n}$ and $\tau_{n}$ with $\tau_{n}$ in decreasing order, i. e., $\tau_{n}>\tau_{n+1}$, for $n=1,2 \cdots, N$. The corresponding radii $R_{n}=C\left(t-\tau_{n}\right)$ are in increasing order. These $N$ points divide $\overline{\mathcal{J}}$ into $N+1$ segments. The segment from point $\bar{K}_{n-1}$ to $\bar{K}_{n}$, is denoted by $\overline{\mathcal{J}}_{n}$ with $\tau_{0}=\tau^{*}$ and $\tau_{N+1}=\tau_{*}$.

If the supersonic interval ends at $t^{*}=t$, we have $R\left(t^{*}\right)=0$. If $t^{*}<t$, the interval ends with $M\left(t^{*}\right)=1$ due to the continuity of $M(\tau)$. For either case, we see from (2.1), (2.2) and (2.3), that 
$\{7 a\}$ For the supersonic region, the critical curve $\overline{\mathcal{J}}$ ends at $\tau=t^{*}$ on the $\bar{\xi}$ axis with $\sigma^{\prime}\left(t^{*}\right)<0$. If $\overline{\mathcal{J}}$ has one or more extremum points, $N \geq 1$, the first one $\bar{K}_{1}$ has to be a local maximum. Therefore, $\bar{K}_{n}$ is a local maximum if $n$ is odd and is a minimum if even.

$\{7 b\}$ If the supersonic interval begins at $t_{*}>0$, we have $M\left(t_{*}\right)=1$ and $\sigma^{\prime}(t) \rightarrow+\infty$ as $t \rightarrow t_{*}+0$. Hence $\bar{K}_{N}$ is a local maximum and $N$ is odd. If $N$ is even, it is necessary that $t_{*}=0$.

If the supersonic interval begins at $t_{*}=0$, we have $M(0) \geq 1$ and the sign of $\sigma^{\prime}\left(0^{+}\right)$depends on the initial acceleration or $M^{\prime}\left(0^{+}\right)$. For example, in the case of constant supersonic speed, $M^{\prime}(\tau)=0$ and hence $\sigma^{\prime}<0$ as shown in Figs. 2. On the other hand, the sign can be positive, if the interval begins with an acceleration at a low supersonic speed. See for example Fig. 4.

Because of $\{7\}$, the number $N$ of extrema of $\overline{\mathcal{J}}$ will be even or odd depending on whether $\sigma^{\prime}\left(t_{*}\right)$ is negative or not. Thus

$\{8 a\}$ If the ordinate of the critical curve $\overline{\mathcal{J}}$ decreases at the beginning of a supersonic interval, i. e., $\sigma^{\prime}\left(t_{*}+0\right)<0, N$ has to be even. The curve has either no extremum or pairs of maxima and minima, with the last one being a local minimum $\sigma_{N}$ at $\bar{K}_{N}$.

$\{8 b\}$ If the ordinate of the critical curve $\overline{\mathcal{J}}$ is not decreasing at the beginning of a supersonic interval, i. e., $\sigma^{\prime}\left(t_{*}+0\right) \geq 0, N$ has to be odd. This is the case when $t_{*}>0$. The curve $\overline{\mathcal{J}}$ has at least one local maximum $\sigma$ at point $\bar{K}_{1}$ and time $\tau_{1}$. If there are additional ones then they come in pairs of minima and maxima. The last one is a local maximum at $\bar{K}_{N}$.

Note that $\{7 b\}$ is included in $\{8 b\}$. Since $\overline{\mathcal{J}}$ has an extremum $\sigma$ only when $M^{\prime}>0,\{8 a\}$ and $\{8 b\}$ are applicable to an accelerating supersonic interval.

From (2.4a) for $\sigma^{\prime \prime}$ at an extremum point and $\{6\}$ that $M^{\prime}>0$, it is clear that $\sigma_{e}^{\prime \prime}<0$ when $M^{\prime \prime} \leq 0$ and the extremum point can not be a local minimum. Therefore

$\{8 c\}$ In a supersonic region with constant or decreasing acceleration, there can be at most one local maximum and no local minimum.

Note that for the case of a constant supersonic speed [3] reviewed briefly in $\S 1.2$, the critical curve $\overline{\mathcal{J}}$ which is a radial line does not have a local extremum $\sigma$. This result is in agreement with $\{8 a\}$ since $\sigma^{\prime}(\tau)=-1 / M, \tau \in[0, t]$. For the case of constantly accelerating speed from subsonic to supersonic [2], reviewed briefly in $\S 1.3$, we can use $\{8 b\}$ and $\{8 c\}$ to conclude that the critical curve $\overline{\mathcal{J}}$ has only one maximum point and no minimum as shown in Fig. 4.

Figure 6 gives another demonstration of $\{8 a\}$. It is the case of accelerating at a constant rate from a low supersonic speed, with $M(\tau)=1.15+\tau^{2}$ for $0 \leq \tau \leq t=2.25$. The critical curve $\overline{\mathcal{J}}$ begins at point $\bar{T}$ on the semicircle $\bar{B}$ with $M(0)=1.15$ and $\sigma(0)=$ $C t \sqrt{1-M^{-2}}=0.494 C t$. As $\tau$ increases, the ordinate $\sigma$ of $\overline{\mathcal{J}}$ decreases to a minimum at point $K_{2}$ with $\tau_{2}=0.0918$, increases to a maximum at point $\bar{K}_{1}$ with $\tau_{1}=0.609$, and then decreases reaching the origin $\bar{P}$ at $\tau=2.25$ with $M=6.2125$. Points $\bar{K}_{1}$ and $\bar{K}_{2}$ divide $\overline{\mathcal{J}}$ into three segments $\overline{\mathcal{J}}_{n}, n=1,2,3$. Similar to Figs. 2 and 4 , curve $\overline{\mathcal{J}}$ partitions the 
domain of dependence $\overline{\mathcal{D}}$ into $\overline{\mathcal{D}}^{+}$and $\overline{\mathcal{D}}^{-}$. Also shown are the partition of $\overline{\mathcal{D}}^{ \pm}$under the rules to be described in $\S 3$.

\subsection{The critical curve $\mathcal{J}$ in the $\xi \sigma$ plane}

Now we study the properties of the image $\mathcal{J}$ in a meridian plane, the $\xi \sigma$ plane with $\sigma \geq 0$. With $\xi$ related to $\bar{\xi}$ by (1.12), (2.2) and (2.3) become the equations for the image $\mathcal{J}$ in the $\xi \sigma$ plane,

$$
\xi=C(t-\tau) / M-C \int_{\tau}^{t} M\left(\tau^{\prime}\right) d \tau^{\prime}, \quad \sigma=C(t-\tau) \sqrt{M^{2}-1} / M
$$

and

$$
\frac{d \xi}{d \tau}=-\frac{C M+R M^{\prime}}{M^{2}}+C M=-\frac{d \sigma}{d \tau} \sqrt{M^{2}-1} \quad \text { hence } \quad \frac{d \sigma}{d \xi}=-\tan \alpha
$$

Thus the tangent line to the curve $\mathcal{J}$ is a backward Mach line,

$$
\sigma-R \cos \alpha=-\tan \alpha\{\xi-[X(\tau)-X(t)+R \sin \alpha]\}
$$

Note that the image of a semicircle $R=C(t-\tau)$ in the $\bar{\xi} \sigma$ plane is a semicircle of the same radius in the $\xi \sigma$ plane centered at $(-X(t)+X(\tau), 0)$,

$$
[\xi+X(t)-X(\tau)]^{2}+\sigma^{2}=C^{2}(t-\tau)^{2}
$$

From (2.7) and (2.8), we see that

$\{9\}$ The curve $\mathcal{J}$ in the $\xi \sigma$ plane is the envelope of the family of Mach lines (2.7) and also of the family of circles (2.8) with parameter $\tau \in\left(t_{*}, t^{*}\right)$.

Figure 3 shows the images of curves in the $\bar{\xi} \sigma$ plane shown in Fig. 2. At a constant supersonic speed, the curve $\mathcal{J}$ becomes the backward Mach line TP enveloping the family of circles (2.8). Figure 5 shows the images of curves in Fig. 4 for a constant accelerating motion. The curve $\mathcal{J}$ begins at point $T$ at $\tau=1$ and $M=1$ with a vertical tangent corresponding to the back facing Mach angle $\pi-\alpha=\pi / 2$. The curve approaches the origin $P$ as $\tau \rightarrow t$ along a radial line which is the backward Mach line. Tangent lines to $\mathcal{J}$ or the local backward Mach lines are shown in the insert. Figure 7 shows the images of curves in Fig. 6 for a constant rate acceleration from a low supersonic Mach number. The semicircle $\mathcal{B}$ with radius $C t$ and centered at $(-X(t), 0)$ is the image of the circular boundary $\overline{\mathcal{B}}$ of $\overline{\mathcal{D}}$ when $\tau=0$. The critical curve $\mathcal{J}$ is divided by the point $K_{1}$ of local maximum and the point $K_{2}$ of local minimum into three branches. Se also the insert. The first branch $\mathcal{J}_{1}$ starts at the origin $P$ along the radial line or the the barh ward Mach line at $\tau=t$. The third branch $\mathcal{J}_{3}$ ends at point $T$ on the semicircle $\mathcal{E}$ with a common tangent; the backward Mach line at $\tau=0$ with $M=1.15$.

Using (2.6), we obtain the curvature of $\mathcal{J}$,

$$
\kappa=\frac{M_{\xi}}{M^{2}}=-\frac{M^{\prime}(\tau)}{\sigma^{\prime}(\tau) M^{2} \sqrt{M^{2}-1}}
$$


The sign of $\kappa$ depends on the sign of $M^{\prime} / \sigma^{\prime}$. From $\{6\}$, we know that at an extremum point on $\overline{\mathcal{J}}, \sigma^{\prime}=0, M^{\prime}>0$ and (2.9) says that the radius of curvature $1 / \kappa$ at the image point on $\mathcal{J}$ vanishes. If the extremum point on $\overline{\mathcal{J}}$ is either a local maximum or minimum, say point $\bar{K}_{n}$ at $\tau_{n}$, then $\sigma^{\prime}$ and $1 / \kappa$ change sign along $\mathcal{J}$ as $\tau$ passes $\tau_{n}$. The point $K_{n}$, which is the image of a local maximum or minimum point $\bar{K}_{n}$ on $\overline{\mathcal{J}}$, is a cusp on $\mathcal{J}$ formed by two adjacent branches $\mathcal{J}_{n}$ and $\mathcal{J}_{n+1}$.

Now we study the curve $\mathcal{J}$ or rather the two adjacent branches in the neighborhood of the cusp $K_{n}$. From (2.3) and (2.9), we see that on these two branches, $\sigma^{\prime}, \xi^{\prime}$ and $1 / \kappa$ are of opposite sign and they vanish at $K_{n}$. With $M^{\prime}>0$, the slope of $\mathcal{J}$ is increasing continuously as $\tau$ increases across $\tau_{n}$ from branch $\mathcal{J}_{n}$ to $\mathcal{J}_{n+1}$. We describe the curve $\mathcal{J}$ in the neighborhood of a cusp $K_{n}$,

$\{10 a\}$ The image of a local maximum (minimum) point $\bar{K}_{n}$ on $\overline{\mathcal{J}}$ is also a local maximum (minimum) point on $\overline{\mathcal{J}}$. The segments $\overline{\mathcal{J}}_{n}$ and $\overline{\mathcal{J}}_{n+1}$ on the left and right of point $\bar{K}_{n}$ are mapped onto the right and left branches $\mathcal{J}_{n}$ and $\mathcal{J}_{n+1}$ of the cusp $K_{n}$.

$\{10 b\}$ As $\tau$ increases across $\tau_{n}$, the slope of the tangent line or the backward Mach line (2.6) increases along branch $\mathcal{J}_{n+1}$ to the cusp $K_{n}$ and then continues along branch $\mathcal{J}_{n}$. If $\sigma$ attains a local maximum (minimum) at cusp $K_{n}, \xi$ reaches its minimum (maximum).

$\{10 c\}$ The branch $\mathcal{J}_{n+1}$ is concave downward (upward) and lies below (above) $\mathcal{J}_{n}$ when the cusp $K_{n}$ is a local maximum (minimum) point. The two branches of opposite concavity form a simple cusp of the first species.

From (2.3a) and (2.9), we know that if $M^{\prime}=0$, then $\sigma^{\prime}<0$ and $\kappa=0$. and that the curvature of a branch can change sign if $M^{\prime}$ changes sign. Also we see that $M^{\prime}$ has to be positive, when $\sigma^{\prime} \geq 0$. Therefore,

\{11a\} A point on $\mathcal{J}$, where the Mach number is a local maximum or minimum, is a point of inflection of $\mathcal{J}$. The curvature of $\mathcal{J}$ changes sign whenever the velocity changes from decelerating to accelerating or vice versa.

\{11b\} A branch joining a point of local minimum $\sigma$ to a local maximum, as $\tau$ increases, has to be in an accelerating subregion and has to be concave downward. A decelerating interval, $M^{\prime}<0$, can occur only on a branch joining a local maximum to minimum and this branch is concave upward during an accelerating interval but downward in a decelerating interval.

To see the additional partitions needed for the subdomains $\bar{V}^{ \pm}$, so that the mapping (1.12) of each subdomain on to its image is one to one, we study the mapping of a horizontal segment $\vec{S}_{r} \vec{S}_{l}$ of constant $\sigma_{c}$ in the $\bar{\xi} \sigma$ plane to its image $S_{r} S_{l}$ in the $\xi \sigma$ plane. If the segment $\bar{S}_{r} \bar{S}_{l}$ lies either in $\bar{V}^{+}$or in $\bar{V}^{-}$, i. e., the segment does not intersect the critical curve $\overline{\mathcal{J}}$, the Jacobian $J=d \bar{\xi} / d \xi,(1.13)$, does not change sign and the mapping is one to one. We need to study only those segments crossing $\overline{\mathcal{J}}$, on which $J=0$ or $\cos \psi=\sqrt{M^{2}-1} / M \geq 0$. Therefore, we consider the segments with $\bar{\xi} \geq 0$ that cut two adjacent branches of the critical curve $\overline{\mathcal{J}}$, the left branch $\overline{\mathcal{J}}_{n}$ and the right branch $\overline{\mathcal{J}}_{n+1}$, at $\bar{S}_{n}$ and $\bar{S}_{n+1}$ as shown in Fig. 8. In Fig. 8a (8b), the point $\bar{K}_{n}$ is a local maximum (minimum) and $\sigma_{c}$ is less (greater) than $\sigma_{n}$. The segment $\bar{S}_{r} \bar{S}_{l}$ is divided into three segments $\bar{S}_{r} \bar{S}_{n+1}, \bar{S}_{n+1} \bar{S}_{n}$ and $\bar{S}_{n} \bar{S}_{l}$. They are oriented as indicated by the arrow in the direction opposite to the $\bar{\xi}$ axis as $\tau$ increases, because $\bar{\xi}^{\prime}=-C R / \bar{\xi} \leq 0$. Since $\pm d \xi / d \bar{\xi}>0$ in $\bar{V}^{ \pm}$, the image of the segment 
of $\bar{S}_{r} \bar{S}_{l}$ in $\bar{V}^{ \pm}$is oriented in the direction of $\mp \xi$ axis as shown in Fig. 8. Note that the two image branches $\mathcal{J}_{n}$ and $\mathcal{J}_{n+1}$ form a cusp at $K_{n}$ with branch $\mathcal{J}_{n}$ lying to the right (left) of $\mathcal{J}_{n+1}$. in Fig. 8a (8b). The first and third segments $\bar{S}_{r} \bar{S}_{n+1}$ and $\bar{S}_{n} \bar{S}_{l}$ lie above (below) $\overline{\mathcal{J}}$ therefore, their images $S_{r} S_{n+1}$ and $S_{n} S_{l}$ are oriented in the same (opposite) direction from right to left (from left to right). The second segment $\bar{S}_{n+1} \bar{S}_{n}$ lies below (above) $\overline{\mathcal{J}}$, therefore its image $S_{n+1} S_{n}$ is oriented in the opposite (same) direction from right to left (from left to right). Because of the change of direction of the image segments across each branch, the second segment $S_{n+1} S_{n}$ and the two branches $\mathcal{J}_{n}$ and $\mathcal{J}_{n+1}$ lie on the same sheet while the first and third segments lie on two different sheets. Therefore, the first segment $S_{r} S_{n+1}$ will be continued across the branch $\mathcal{J}_{n}$ without a reversal of direction and the third one $S_{n} S_{l}$ across $\mathcal{J}_{n+1}$.

Consider the inverse transformation of (1.12) of a point in the $\xi \sigma$ plane without considering the three separate sheets. A point $q$ in the middle segment $S_{n+1} S_{n}$ has at least three image points $\bar{q}_{r}, \bar{q}$ and $\bar{q}_{l}$ which lie in the segments $\bar{S}_{r} \bar{S}_{n+1}, \bar{S}_{n+1} \bar{S}_{n}$ and $\bar{S}_{n} \bar{S}_{l}$ respectively. As point $q$ approaches an end point say $S_{n}$, the two image points $\bar{q}_{l}$ and $\bar{q}$ approach each other and coincide at $\bar{S}_{n}$ as a double root. The third image point $\bar{q}_{r}$ approaches a point $\bar{q}^{*}$ to the right of $\bar{S}_{n+1}$. When $q$ crosses over to the right of $S_{n}$, there is only one image point $\bar{q}_{l}$ which moves to the right of the point $\bar{q}^{*}$. We say that

$\{12 a\}$ A horizontal segment $\bar{S}_{r} \bar{S}_{l}$ intersecting $\overline{\mathcal{J}}$ twice at points $\bar{S}_{n}$ and $\bar{S}_{n+1}$ is divided by $\overline{\mathcal{J}}$ into three nonoverlapping horizontal segments $\bar{S}_{r} \bar{S}_{n+1}, \bar{S}_{n+1} \bar{S}_{n}$ and $\bar{S}_{n} \bar{S}_{l}$ oriented in the same direction. They are mapped respectively onto three segments $S_{r} S_{n+1}$, $S_{n+1} S_{n}$ and $S_{n} S_{l}$ with folding or change of orientation at the points $S_{n}$ and $S_{n+1}$ on the branches $\mathcal{J}_{n}$ and $\mathcal{J}_{n+1}$ respectively.

$\{12 b\}$ The segments $S_{r} S_{n+1}$, and $S_{n} S_{l}$ overlap each other over the segment $S_{n+1} S_{n}$. A point $S$ on $S_{n+1} S_{n}$ lies on all three segments; therefore, the multiplicity of point $S$ is three if the inverse mapping is restricted to the segment $\bar{S}_{r} \bar{S}_{l}$. If point $S$ lies on either $S_{r} S_{n+1}$ or $S_{n} S_{l}$, the multiplicity of $S$ is one.

To end this section, we recall that a critical point $\bar{K}_{e}$ on $\overline{\mathcal{J}}$ which is neither a local minimum nor maximum is excluded in the above discussion because the image of $\bar{K}_{e}$ is not a cusp and behaves the same as a point on $\mathcal{J}$ with $\sigma^{\prime} \neq 0$. If a parameter $\lambda$ (or parameters) defining $U(\tau)$ can be varied so that two adjacent roots $\tau_{n}$ and $\tau_{n+1}$ of $\sigma^{\prime}=0$ for $\lambda<\lambda_{e}$ become a double root $\tau_{e}$ at $\lambda=\lambda_{e}$ and disappear for $\lambda>\lambda_{e}$, then the extremum point at the double root $\tau_{e}$ is neither a maximum nor a minimum. There is no cusp formation in the mapping of the neighborhood of point $\bar{K}_{e}$ at $\tau_{e}$ when the parameter $\lambda \geq \lambda_{e}$. A small decrease in $\lambda$ from $\lambda_{e}$ results in two cusps appearing like a butterfly [4] in the mapping of the neighborhood of $\bar{K}_{e}$. There is an increase in multiplicity of 2 . The sensitivity of the mapping on the parameter has been known in many other physical problems [5] and will not be addressed here.

As a complement to the above quantitative analysis, we present in Appendix B the qualitative analysis of the inverse transformation of (1.12) and derive the formula for the retarded time for a point in the neighborhood of a branch near or away from a cusp. In $\S 3$, we use the above statements $\{1\}$ to $\{12\}$ to formulate the rules for the partition of the domain of dependence $\overline{\mathcal{D}}$. 


\section{The Rules for the Partition of the Domain of Dependence}

Guided by the statements $\{1\}$ to $\{12\}$ in $\$ 2$, we formulate the general rules for the partition of of the domain of dependence $\overline{\mathcal{D}}$ for a medium moving at an unsteady velocity $U(\tau) \hat{\imath}$. Since the multiplicity of $\overline{\mathcal{D}}$ is 1 , we seek to partition $\overline{\mathcal{D}}$ into $I$ nonoverlapping subdomains $\bar{V}_{i}$ such that the mapping from $\bar{V}_{i}$ to $V_{i}$ in the $\xi \sigma$ plane by (1.12) is one to one. Hence the multiplicity of $V_{i}$ is 1 , i. e., for each point in $V_{i}$ there is only one retarded time. Also we define the minimum number of partitions $I$.

Since different supersonic intervals in $[0, t]$ are separated by subsonic intervals, we can study the partition of the domain of dependence for each supersonic region one by one. Therefore, it suffices to study the case where there is only one supersonic interval $\left(t_{*}, t^{*}\right)$. The Jacobian $J$ of transformation (1.12) changes sign in the supersonic region across the critical curve $\overline{\mathcal{J}}$ on which $J=0$. The curve ends at $\tau=t^{*}$ on the $\bar{\xi}$ axis. It begins at $\tau=t_{*}$ from the $\bar{\xi}$ axis if $t_{*}>0$ or from the semi-circular boundary $\overline{\mathcal{B}}$ if $t_{*}=0$. Therefore, the critical curve $\overline{\mathcal{J}}$ partitions $\overline{\mathcal{D}}$ into two nonoverlapping regions $\bar{V}^{ \pm}$where $\pm J>0$. The region $\bar{V}^{-}$is bounded below by the $\bar{\xi}$ axis and above by the the critical curve $\overline{\mathcal{J}}$ if $t_{*}>0$ and in addition partially by $\overline{\mathcal{B}}$ if $t_{*}=0$. From (2.1) we see that $\overline{\mathcal{J}}$ and hence $\bar{V}^{-}$lie inside the circular sector $0 \leq \psi \leq \psi_{m}$ where $\psi_{m}=\arccos \left[1 / M_{m a x}\right]$ and $M_{m a x}$ is the maximum of $M(\tau)$.

From $\{12 a\}$ and $\{12 b\}$, we see that the mapping from $\bar{V}^{ \pm}$to their images $V^{ \pm}$may not be one to one. Further partitions of $\bar{V}^{ \pm}$are needed to separate the region in $\bar{V}^{+}$ above $\left(\bar{V}^{-}\right.$below) the curve $\overline{\mathcal{J}}$ to the left hand side of a peak (valley), a local maximum (minimum) point $\bar{K}_{n}$ at instant $\tau_{n}$ where $n$ is odd (even), from the right hand side of the peak (valley). There is not a unique way to do the separation. We choose to partition $\bar{V}^{+}$ $\left(\bar{V}^{-}\right)$by the large (small) circular arc $\overline{\mathcal{C}}_{n}^{+}\left(\overline{\mathcal{C}}_{n}^{-}\right)$passing through the point $\bar{K}_{n}$ with radius $C\left(t-\tau_{n}\right)$ at the retarded time $\tau_{n}$ for $n$ odd (even).

Before we state the rules for the partition of the domain of dependence, $\overline{\mathcal{D}}$, for a given velocity function $U(\tau), \tau \in[0, t]$ with a supersonic interval, we carry out the following two preparatory steps.

Step 1. Graph the boundary of the domain of dependence $\overline{\mathcal{D}}$ in the $\bar{\xi} \sigma$ meridian plane, bounded by a semi-circle $\overline{\mathcal{B}}$ with radius $C t$ centered at the origin, and then the critical curve $\overline{\mathcal{J}}$ defined by (2.2). Locate the roots, of $\sigma^{\prime}=0$ in (2.3a) while $\sigma^{\prime \prime} \neq 0$ and arrange the roots, $\tau_{n}, n=1,2 \cdots, N$ in decreasing order of $\tau$, i. e., $\tau_{n}>\tau_{n+1}$. These $N$ points divide $\overline{\mathcal{J}}$ into $N+1$ segments $\overline{\mathcal{J}}_{j}, j=1, \cdots, N+1$. Segment $\overline{\mathcal{J}}_{j}$ joins points $\bar{K}_{j-1}$ and $\bar{K}_{j}$. Here $\bar{K}_{0}$ and $\bar{K}_{N+2}$ denote the end points of $\overline{\mathcal{J}}$ at $\tau=t^{*}$ and $\tau_{*}$.

In this step we made use of $\{6\}$ and $\{7\}$ and note that these roots should be in accelerating supersonic interval(s). The first segment $\overline{\mathcal{J}}_{1}$ joins the end point at $\tau=t^{*}$ on the $\bar{\xi}$ axis to the local maximum point $\bar{K}_{1}$. When $\tau_{*}>0$, the last segment $\overline{\mathcal{J}}_{N+1}$ joins print $\bar{K}_{N}$ to the end point $\bar{K}_{N+1}$ on the $\bar{\xi}$ axis. Hence $\bar{K}_{N}$ is a local maximum and $X$ has 10 be odd. If $\tau_{*}=0$, the end point $\bar{K}_{N+2}$ lies on the semicircle $\overline{\mathcal{B}}$ of $\tau=0 . \bar{K}_{N}$ is a local maximum (minimum) if $N$ is odd (even) while $N$ is odd (even) if $\sigma^{\prime}(0)$ is positive (nngative). Let $L^{+}$and $L^{-}$denote the number of maximum and minimum points on $\overline{\mathcal{J}}$, then we have $L^{+}+L^{-}=N$ and

$$
L^{+}=L^{-}+1=(N+1) / 2 \quad \text { when } N \text { is odd }
$$




$$
L^{+}=L^{-}=N / 2 \quad \text { when } N \text { is even. }
$$

Step 2. Graph the images of the boundary curve $\overline{\mathcal{B}}$ and the critical curve $\overline{\mathcal{J}}$ in the $\xi \sigma$ plane. The image $\mathcal{B}$ is a semi-circle of radius $C t$ centered at $(-X(t), 0)$. The image $\mathcal{J}$ forms cusps at the $N$ image points $K_{n}$.

These $N$ cusps divide $\mathcal{J}$ into $N+1$ branches, $\mathcal{J}_{n}$, the images of the segments $\overline{\mathcal{J}}_{n}$. The domain od dependence $\mathcal{D}$ is the maximum area bounded above by either $\mathcal{B}$ or $\mathcal{J}$ and below by the $\xi$ axis.

Now we state the rules of partition:

Rule 1. Partition $\overline{\mathcal{D}}$ by the critical curve $\overline{\mathcal{J}}$ into $\bar{V}^{ \pm}$where $\pm J>0$

The domain $\overline{\mathcal{D}}^{-}$is bounded below by the $\bar{\xi}$ axis and above by $\overline{\mathcal{J}}$ if the velocity begins subsonic $M(0)<1$ and becomes sonic at $\tau=t_{*}$. If the velocity is supersonic at $\tau_{*}=0$ the end point $T$ or $\bar{K}_{N+1}$ lies on the semicircle $\overline{\mathcal{B}}$ with polar angle $\psi_{N+1}=\arccos 1 / M(0)$ and then $\overline{\mathcal{D}}^{-}$is bounded above in addition by the small arc $\overline{\mathcal{C}}_{\bar{N}+1}^{-}$of the semicircle $\overline{\mathcal{B}}$ with $0 \leq \psi \leq \psi_{N+1}$. Note that the large arc $\overline{\mathcal{C}}_{N+1}^{+}$of $\overline{\mathcal{B}}$, with $\psi_{N+1} \leq \psi \leq \pi$, is a boundary of $\overline{\bar{V}}+$. A semicircle of constant $\tau$ in the supersonic region is divided by the critical curve into two arcs $\overline{\mathcal{C}}^{ \pm}$in $\bar{V}^{ \pm}$. The arc $\overline{\mathcal{C}}^{-}$is the smaller one since its central angle is $\arccos 1 / M<\pi / 2$. The subscript $n$ will be added to $\overline{\mathcal{C}}^{ \pm}$when $\tau=\tau_{n}, n=1, \cdots, N+1$, with $\tau_{N+1}$ equal to $t_{*}$.

Rule 2a. Partition $\bar{V}^{+}$into $1+L^{+}$nonoverlapping domains $\vec{V}_{2 l-1}^{+}, l=1,2, \cdots, 1+L^{+}$, by the $L^{+}$large circular arcs, $\overline{\mathcal{C}}_{2 l-1}^{+}$, with constant retarded time $\tau_{2 l-1}$ passing through the local maximum point $\bar{K}_{2 l-1}$ on $\overline{\mathcal{J}}$.

Rule 2b. Partition $\bar{V}^{-}$into $1+L^{-}$nonoverlapping domains $\bar{V}_{2 l}^{-}, l=l, 2, \cdots, 1+L^{-}$, by the $L^{-}$large circular arcs, $\overline{\mathcal{C}}_{2 l}^{-}$, with constant retarded time $\tau_{2 l}$ passing through the local maximum point $\bar{K}_{2 l}$ on $\overline{\mathcal{J}}$.

The subdomains and circular arcs in $\overline{\mathcal{D}}^{ \pm}$are numbered so that they can be grouped together as $N+2$ subdomains $\bar{V}_{i}^{ \pm}$and $N+1$ circular arcs $\overline{\mathcal{C}}_{i}^{ \pm}$at $\tau_{i}$ using the plus or minus superscript for $i$ odd or even. Thus the domain of independence $\overline{\mathcal{D}}$ is partitioned into $I$ nonoverlapping subdomains $\bar{V}_{i}^{ \pm}$, where

$$
I=\left(1+L^{+}\right)+\left(1+L^{-}\right)=2+N
$$

is the minimum number.

A subdomain $\bar{V}_{i}^{ \pm}$is bounded by the $\bar{\xi}$ axis, two circular arcs $\overline{\mathcal{C}}_{i-2}^{ \pm}, \overline{\mathcal{C}}_{i}^{ \pm}$and two segments of the critical curve $\overline{\mathcal{J}}_{i-1}^{ \pm}$and $\overline{\mathcal{J}}_{i}^{ \pm}$. Here, the segment $\overline{\mathcal{J}}_{0}$ reduces to the end point $\bar{K}_{0}$ of $\overline{\mathcal{J}}$ and the $\operatorname{arc} \overline{\mathcal{C}}_{i}^{ \pm}$is empty when $i \leq 0$.

Rule 3. Find the images of the $I$ nonoverlapping subdomains $\bar{V}_{i}^{ \pm}$in the $\xi \sigma$ meridian plane one by one and denote the images by $V_{i}^{ \pm}$using the plus or the minus superscript for $i$ odd or even. The multiplicity of $V_{i}^{ \pm}$is one.

Since the mapping (1.12) is independent of the radial and polar coordinates, $\sigma$ and $\theta$, we can now define the volumes of integration of the integral in (1.16) in the $\xi, \eta, \zeta$ space. 
Rule 4. Rotate each one of the $N+2$ subdomains $V_{i}^{ \pm}$in the meridian plane about the $\xi$ axis to generate a volume of revolution in $\xi, \eta, \zeta$ space and denote the volume by the same symbol $V_{i}^{ \pm}$. The volume integral in (1.16) for the acoustic potential in the $\mathbf{x}$ space is expressed as the sum of the $N+2$ integrals over the volumes $V_{i}^{ \pm}$. The multiplicity of each volume is one

In case that there is more than one supersonic interval in $[0, t]$, say $s$ of them, we apply the above rules to these regions one by one. We locate the local maximum and minimum on the critical curves in the regions, draw the corresponding partition circular arcs in the $\bar{\xi} \sigma$ plane and find the images in the $\xi \sigma$ plane. The total number $I$ of nonoverlapping domains in the $\bar{\xi} \sigma$ plane or the number of domains of integration in the $\xi \sigma$ plane is

$$
I=1+s+N,
$$

where $N$ denotes the total number of maxima and minima on the $s$ critical curves. When $s=1$, we recover $(3.3 \mathrm{a})$.

A formula or a numerical scheme is needed for the inverse solution of (1.12) to determine the retarded time $\tau$ for a point $S$ in a $V_{j}^{ \pm}$. Consider a point $S_{0}\left(\xi_{0}, \sigma_{0}\right)$ in a domain $V_{i}^{ \pm}$defined under Rule 2a or b. Let points $S_{l}$ and $S_{r}$ denote the left and right end points of the horizontal segment in the domain passing through point $S_{0}$. Each end point will either be on a circular arc of constant $\tau$ or a branch of the critical curve on which $\sigma^{\prime} \neq 0$. Thus we know the values of $\tau$ and $\xi$ and $\bar{\xi}$ for both end points. Let us use the subscripts $l$ and $r$ to denote the values associated with points $S_{l}$ and $S_{r}$. If point $S_{0}$ coincides with one of the end points, the inverse solution of (1.12) is found. If $S_{0}$ is not an end point, $J=d \xi / d \bar{\xi} \neq 0$ in the segment, hence there is a unique inverse solution $\bar{\xi}_{0}$. Now we can determine the numerical solution $\bar{\xi}_{0}$ of (1.12) by Newton's method using the linear interpolation formula from the end points to get the first estimate for $\bar{\xi}_{0}$. If the point $S_{0}$ is close to a cusp of the region or to an end point on a branch of the critical curve, we shall compute the first estimate of $\tau_{0}$ by the approximate formula for the roots of (1.12) presented in Appendix $\mathrm{C}$ choosing the root lying in between $\bar{\xi}_{l}$ and $\bar{\xi}_{r}$. Thus we have a procedure for the determination of the retarded time $\tau_{0}$ and provide a quantitative proof that the multiplicity of point $S_{0}$ in a domain $V_{i}^{ \pm}$is one.

Now we are ready to express the integral in $(1.16 \mathrm{~b})$ for the velocity potential at $P(x, y, z)$ as the sum of integrals over the volumes of revolution $V_{i}$, under Rule 4,

$$
\Phi(t, x, y, z)=-\frac{1}{4 \pi} \sum_{i=1}^{I} \iint_{V_{i}} \sigma d \sigma d \xi\left[\int_{0}^{2 \pi} d \theta \frac{q(\tau, x+\xi, y+\sigma \cos \theta, z+\sigma \sin \theta)}{\left|R_{i}-M_{i} \bar{\xi}_{i}\right|}\right]
$$

In (3.4), $V_{i}$ denotes a subdomain in the meridian plane under Rule 3. For each $V_{i}, \tau_{i}$ and hence $R_{i}, M_{i}$ and $\bar{\xi}_{i}$ are functions of $\xi, \sigma$ and $t$ independent of $\theta$. This formula does not need the knowledge of multiplicity.

To demonstrate the meaning of multiplicity and the application of (3.4), we consider the source distribution to be a point source of unit strength created at point $S\left(x^{\prime}, y^{\prime}, z^{\prime}\right)$ for $\tau>0$. If $S$ lies in $V_{i_{1}}, \cdots, V_{i_{m}}$, with $i_{j} \in[1, I]$ and multiplicity $m \leq I$, then the intensity 
received at $P$ is

$$
\Phi(t, x, y, z)=-\sum_{j=1}^{m} 1 /\left\{4 \pi\left|R_{i j}-M_{i_{j}} \bar{\xi}_{i_{j}}\right|\right\} .
$$

Note that the domain of dependence $\mathcal{D}$ of point $P(x, y, z)$ and the associated subdomains $V_{j}$ are $t$-dependent. They were empty for $t<0$, grow in size and change in shape as time $t$ increases. Consequently, the multiplicity of point $S$ is also $t$-dependent. The multiplicity changes by an integer and results in the addition or removal of term(s) on the right hand side of (3.5) and a discontinuity of the intensity at $P$.

Now we formulate the rules for the change of multiplicity of point $S$, or point $S(\xi, \sigma)$ in

a meridian plane, when it enters or leaves a subdomain $V_{i}^{ \pm}$. Besides the axis of symmetry, the $\xi$ axis, a subdomain is bounded by a segment of the semi-circle $\mathcal{B}$, branch(es) of the critical curve $\mathcal{J}$ and/or circular $\operatorname{arc}(\mathrm{s}) \mathcal{C}_{i}^{ \pm}, i \leq N$, partitioning $V^{ \pm}$. These three types of boundaries, which are moving, are called wave fronts.

The multiplicity $m(t)$ of point $S$ begins with zero at $t=0$. If point $S$ remains outside of $\mathcal{D}$ for all $t$, we have $m(t) \equiv 0$. The multiplicity changes when point $S$ crosses a wave front in entering the domain of dependence $\mathcal{D}$ or rather a subdomain. The rules for crossing the three types of boundaries are different and hence are stated separately.

Rule $\mathcal{B}$. When $S$ crosses $\mathcal{B}$ to enter (leave) the subdomain $V^{+}$or $V^{-}$of $\mathcal{D}$, the multiplicity $m$ increases(decreases) by 1 .

Rule $\mathcal{J}$. When $S$ crosses a branch of $\mathcal{J}$ with $\sigma^{\prime}(\tau)>0$ from the left to the right hand side, the multiplicity $m$ increases by 2 , and with $\sigma^{\prime}<0, m$ decreases by 2 . When the direction of crossing is reversed the change of $m$ is reversed.

Rule $\mathcal{C}$. When $S$ crosses a circular arc, $\mathcal{C}_{i}^{ \pm}, i \leq N$, partitioning $V^{ \pm}$, there is no change of multiplicity, because the Jacobian $J$ does not change sign and $S$ is entering one subdomain while leaving another.

These three rules can be applied to determine the multiplicity of point $S\left(\xi_{1}, \sigma_{1}\right)$ in $\mathcal{D}$ at an instant $t$, by moving from a point $S_{0}\left(\xi_{0}, \sigma_{0}\right)$ with known $m_{0}$, along a path to $S_{1}$ and counting the changes of multiplicity along the path. For example, we can pick a point $S_{0}$ outside of $\mathcal{D}$ with $\sigma_{0}=\sigma_{1}$ and hence $m_{0}=0$ and the path to $S_{1}$ is horizontal.

In the next section we shall demonstrate the applications of the rules of partition and counting of multiplicity for various unsteady velocities $U(\tau)$.

\section{Examples}

For the cases where the velocity $U(\tau) \hat{\imath}$ of the medium relative to the coordinate system $\mathbf{x}$ remains subsonic [2], [3], the Jacobian of the transformation of $\overline{\mathcal{D}}$ to $\mathcal{D}$ remains positive, and the multiplicity of the domain of dependence $\mathcal{D}$ in $\mathbf{x}$ is equal to one. These cases are examples of the class $I=1$, where $I$ is the minimum number of nonoverlapping domains in $\overline{\mathcal{D}}$. In the following, we present examples having maximum multiplicity greater than or equal to 2 . These examples must have a critical curve, where $J=0$, and hence a supersonic interval, $\left(t_{*}, t^{*}\right)$ in $(0, t)$. See $\{2\}$.

It was stated in $\{1\}$ and $\{2\}$, that the domain of dependence $\overline{\mathcal{D}}$ in the coordinate system $\overline{\mathbf{x}}$, with the medium at rest, is independent of the velocity of the medium relative to 
the system $\mathbf{x}$, the critical curve $\overline{\mathcal{J}}$ is dependent on the velocity. The parametric equations for $\overline{\mathcal{J}}$ in a meridian $\bar{\xi} \sigma$ plane are given by (2.2). The first step is to locate the local maximum and minimum points on the curve $\overline{\mathcal{J}}$, i. e., to locate the roots of $\sigma^{\prime}(\tau)=0$, (2.3a), with $\sigma^{\prime \prime} \neq 0$, which are the roots of

$$
F(\tau)=(t-\tau) M^{\prime}-M\left(M^{2}-1\right)=0, \quad \tau \in(0, t),
$$

with

$$
F^{\prime}(\tau)=(t-\tau) M^{\prime \prime}-3 M^{2} M^{\prime} \neq 0 .
$$

Here we set $C=1$ and hence $M=U(\tau)$. To demonstrate the rules of partition and counting in $\S 3$, we present first in $\S 4.1$ to $\S 4.5$ examples with only one supersonic interval in $[0, t]$ i. e., $s=1$, and then in $\$ 4.6$ and $\S 4.7$ examples with more than one supersonic interval, $s \geq 2$. In the last two subsections there are also examples showing that the maximum multiplicity in $\mathcal{D}$ can be less than the number of partitions or the number of domains of integrations in (3.4), i. e., $m \leq I$. With $s \geq 1$ and $N \geq 0$, we have $I=1+s+N \geq 2$. We begin in $\S 4.1$ with $I=2$.

4.1. Class $I=2$, the critical curve $\bar{J}$ has no local maximum. From $\{7\}$, we see that the curve does not have a local maximum or minimum. Thus (4.1) has no root, i. e., $N=0$. The simplest example is that of constant supersonic speed [3], $U^{\prime}=M^{\prime}=0$ and $M>1$. In the $\bar{\xi} \sigma$ plane, the critical curve $\overline{\mathcal{J}}$ is a radial line $\bar{P} \bar{T}$ which partitions the domain $\overline{\mathcal{D}}$ into $\bar{V}^{+}$and $\bar{V}^{-}$under Rule 1 . Since $\overline{\mathcal{J}}$ has neither a local maximum nor minimum, further partition of $\bar{V}^{ \pm}$is not needed. In Fig. 2, the coordinates $\bar{\sigma}$ and $\xi$ are scaled by $C t$ and the concentric circles are circles of constant $\tau / t$ with radius $1-(\tau / t)$. The radius of the boundary $\overline{\mathcal{B}}$ is 1 . Thus Fig. 2 remains the same for all $t>0$.

The mapping of Fig. 2 onto the $\xi \sigma$ plane scaled by $C t$ will also be invariant for all $t>0$, as shown in Fig. 3. The superposition of the images $V^{+}$and $V^{-}$yields the domain of dependence $\mathcal{D}$, i. e., $V^{+} \cup V^{-}$. The domain $\mathcal{D}$ is bounded above by the critical curve $P T$, a backward Mach line, and by the large circular arc $Q T$ of the semicircle $\mathcal{B}$ at $\tau=0$ and below by the $\xi$ axis. Also shown are the semicircles at retarded time $\tau \in(0, t)$. These semicircles are divided by their envelope $P T$ into smaller and larger arcs. The family of the larger (smaller) arcs covers $V^{+}\left(V^{-}\right)$. Thus $\mathcal{D}=V^{+}$and the multiplicity of $\mathcal{D} \cap V^{-}$is two and that of $\mathcal{D} \notin V^{-}$is one. Detailed discussion of this partition can be found in [2].

It is well known [3] that the domain of dependence of a point $P(x, y, z)$ with the medium moving at a constant supersonic speed is the backward Mach cone from $P$. Since the source distribution has been turned on all the time, the retarded time is in $(-\infty, t]$. The multiplicity for a point in the cone is always 2 . In the current initial value problem the source distribution is turned on at $\tau=0$, therefore, the retarded time has to be in $[0, t]$ and the domain of dependence shown in Fig. 3 is a finite part of the backward cone with multiplicity 1 or 2 , as defined in the preceding paragraph.

We use this simple example to show the meaning of multiplicity to an observer at $P$. We consider a point source at $S$ of unit strength initiated at $\tau=0$. Since both points $P$ and $S$ are fixed in $\mathbf{x}, S$ is a fixed point in the relative coordinates $\xi, \eta, \zeta$ and in the $\xi, \sigma$ plane. But in Fig. 3, the position of point $S$ is moving along the radial line from point $S(1)$ towards the origin $P$ (shown as the dotted line) with the instantaneous radial 
distance $|S(t) P|$ equal to $|S(1) P| / t$ where $S(1)$ is the position at $t=1$. The point $S(1)$ shown in Fig. 3 is lying inside the backward Mach cone but outside $\mathcal{D}$. That is, at $t=1$ the multiplicity of point $S$ is zero, or the signal from $S$ has not reached $P$. At an instant $t_{a}>1$, the radial line intersects the larger circular arc $Q T$ of $\mathcal{B}$ and enters the region $\mathcal{D} \notin V^{-}$with multiplicity 1. At a later instant $t_{b}>t_{a}$, the radial line intersects the smaller circular arc of $\mathcal{B}$ and enters the region $V^{-}$with multiplicity 2 . The observer receives the signal from $S\left(t_{1}\right)$ with intensity $1 /\left(4 \pi R^{*}\right)$ when $t_{b}>t_{1}>t_{a}$ and with the intensity doubled $1 /\left(2 \pi R^{*}\right)$ when $t>t_{b}$. Here $R^{*}$ denotes the pseudo-distance (1.17).

Additional examples for this class of $I=2$ will be found as "degenerated" cases of the classes of $I>2$ in the following subsections.

4.2. Class $I=3$, the critical curve has one local maximum. The simplest example for this class is the case of constant acceleration from zero to supersonic speed. By choosing the time scale, we can write $M=\tau$ and then the the supersonic interval is from $t_{*}=1$ to $t^{*}=t>1$. Equation (4.1) has one root, $\tau_{1}=t^{1 / 3}$. The curve $\overline{\mathcal{J}}$ begins from the $\bar{\xi}$ axis at $\tau=1$ with $M_{*}=1$ rises to the maximum $\bar{K}_{1}$ at $\tau_{1}=t^{1 / 3}$ and descends to the origin at $\tau=t$. Thus this case belongs to the class of $N=1$ and $I=N+2=3$. Note that we can no longer get a time invariant critical curve and partition of the domain of dependence by using $C t$ as the length scale and $t$ as the scale for $\tau$ as we did for constant supersonic speed in $\$ 4.1$. We note that when $t<1, M<1$ for $\tau \in[0, t], \overline{\mathcal{J}}$ does not exist. When $t>1$, $M>1, \overline{\mathcal{J}}$ exists in the interval $[1, t]$ but $\overline{\mathcal{J}}$ and the interval do not grow proportionately with $t$. We have to construct the critical curve and the partition for a given $t$.

Figure 4 shows the critical curve $\overline{\mathcal{J}}$ for $t=\sqrt{5}$. Besides applying Rule 1 to partition $\overline{\mathcal{D}}$ into $\bar{V}^{+}$and $\bar{V}^{-}$, we have to apply Rule $\mathbf{2 a}$ to partition $\bar{V}^{+}$by the circular arc $\overline{\mathcal{C}}_{1}^{+}$of constant $\tau_{1}$ passing through point $\bar{K}_{1}$ into $\bar{V}_{1}^{+}$and $\bar{V}_{3}^{+}$. Thus $\overline{\mathcal{D}}$ is partitioned into $I=3$ nonoverlapping domains, $\bar{V}_{1}^{+}, \bar{V}_{3}^{+}$and $\bar{V}^{-}$.

The images of the nonoverlapping domains in Fig. 4 are the three domains of integration in $\xi$ and $\sigma$ in (3.4). The superposition of these three domains for $t=\sqrt{5}$ onto the $\xi \sigma$ plane is shown in Fig. 5. The union of these three images gives the domain of dependence $\mathcal{D}$ which is bounded above by the semicircle $\mathcal{B}$ and by branch $\mathcal{J}_{2}$ and below by the $\xi$ axis. Besides the $\xi$-axis, $V^{-}$is bounded by the two branches $\mathcal{J}_{1}$ and $\mathcal{J}_{2}, V_{1}^{+}$by the circular arc $\mathcal{C}_{1}^{+}$and the branch $\mathcal{J}_{1}$ and $V_{3}^{+}$by the semicircle $\mathcal{B}$, the arc $\mathcal{C}_{1}^{+}$and the branch $\mathcal{J}_{2}$. Note that $V^{-} \in V_{1}^{+}, V_{3}^{+} \cap V_{1}^{+} \in V^{-}$and $V^{-} \cap V_{3}^{+} \neq \emptyset$. The multiplicity of $V^{-} \cap V_{3}^{+}$is 3 , that of $V^{-} \notin V_{3}^{+}$is 2 and that of $\mathcal{D} \notin V^{-}$is 1 . More detailed descriptions of these three domains of integrations are given in [2].

We use this example to explain that the domain of dependence $\overline{\mathcal{D}}$, the semicircular disc of radius $t$ in the meridian plane, is the domain for all source fields $\bar{q}(\tau, \bar{x}, y, z)$ in the interval $(0, t)$. But for a given source field, there is an effective domain of dependence which is a subdomain $\overline{\mathcal{D}}$. For example, if the source field is created at a time $b>0$, i. e., $\bar{q} \equiv 0$ for $\tau<b$, then only the part of $\overline{\mathcal{D}}$ where the retarded time $\tau$ lies in the interval $[b, t]$ will contribute to the acoustic field at point $\bar{P}$ at time $t$. This effective domain of dependence $\overline{\mathcal{D}}_{e}$ is the subdomain of $\overline{\mathcal{D}}$ below the semicircle $\overline{\mathcal{B}}_{e}$ of $\tau=b$ or radius $R=t-b$. Thus only the portion of the critical curve $\overline{\mathcal{J}}$ and the subdomains $\bar{V}_{i}^{ \pm}$lying inside this 
semicircular disc $\overline{\mathcal{D}}_{e}$ will be mapped to the $\xi \sigma$ plane. $\dagger$ For the current example, $M(\tau)=\tau$, (4.1) becomes

$$
F(\tau)=t-\tau^{3}=0, \quad \tau \in(b, t),
$$

with $F^{\prime}(\tau)=-3 \tau^{2}<0$. Equation (4.2) has one root $\tau_{1}=t^{1 / 3}$ provided that $b<t^{1 / 3}$.

For $b<1$, the supersonic interval $(1, t)$ is in $(b, t)$. Hence the critical curve $\overline{\mathcal{J}}$ and the large circular $\operatorname{arc} \overline{\mathcal{C}}_{1}^{+}$remain inside $\overline{\mathcal{D}}_{e}$. The subdomains $\bar{V}^{-}$and $\bar{V}_{1}^{+}$shown in Fig. 4 remain unchanged. Only the subdomain $\bar{V}_{3}^{+}$is reduced in size with the semicircular boundary $\overline{\mathcal{B}}$ replaced by a smaller one $\overline{\mathcal{B}}_{e}$. Consequently, the images $\mathcal{J}, V^{-}$and $V_{1}^{+}$in the $\xi \sigma$ plane remain the same as those shown in Fig. 5. The image $V_{3}^{+}$is reduced in size with the boundary $\mathcal{B}$ replaced by $\mathcal{B}_{e}$.

If $\tau_{1}>b>1$, part of the segment $\overline{\mathcal{J}}_{2}$ and the subdomain $\bar{V}_{3}^{+}$in addition to that of $\bar{V}^{-}$in Fig. 4 are outside of $\overline{\mathcal{D}}_{e}$ or cut off by the semicircle $\overline{\mathcal{B}}_{e}$ and their images will be cut off in Fig.5. Only the image $V_{1}^{+}$remains unchanged.

When $b=\tau_{1}$, the semicircle $\overline{\mathcal{B}}_{e}$ passes through point $\bar{K}_{1}$, coincides with the semicircle $\overline{\mathcal{C}}_{1}$ at $\tau_{1}$ and completely cuts off the segment $\overline{\mathcal{J}}_{2}$ and the subdomain $\bar{V}_{3}^{+}$in Fig. 4. Thus the critical curve $\overline{\mathcal{J}}_{e}=\overline{\mathcal{J}}_{1}$ does not have a local maximum. The domain of dependence $\mathcal{D}_{e}$ in the $\xi \sigma$ plane is equal to $V_{1}^{+}$in Fig. 5 . The image $V_{e}^{-}$is bounded by the $\xi$-axis, $\mathcal{J}_{2}$ and the small circular arc $\mathcal{C}_{1}^{-}$which together with the arc $\mathcal{C}_{1}^{+}$form the semicircle $\mathcal{B}_{e}$.

When $t>b>\tau_{1}$, the semicircle $\overline{\mathcal{B}}_{e}$ not only cuts off the entire branch $\overline{\mathcal{J}}_{2}$, the circular arc $\overline{\mathcal{C}}_{1}^{+}$and the domain $\bar{V}_{3}^{+}$but also parts of the branch $\overline{\mathcal{J}}_{1}$ and the domains $\bar{V}^{-}$and $\bar{V}_{1}^{+}$. The critical curve $\overline{\mathcal{J}}_{e}$, which is the remaining part of $\mathcal{J}_{1}$, decreases monotonically to the $\bar{\xi}$ axis as $\tau$ increase from $b$ to $t$, i. e., (4.2) has no root. Thus our example degenerates to the class of $I=2$ when $t>b \geq \tau_{1}$.

For a fixed $b>1,(4.2)$ defines a critical time $t_{c}=b^{3}$ for which $F(b)=0, t_{c}=\tau_{1}$ and (4.2) has no root. As $t$ increases over $t_{c}, I$ jumps from 2 to 3 . That is, an observer at point $P$ will receive signals from point $S$, fixed in the coordinate system $\mathbf{x}$, twice if $S \in V^{-}$when $t \leq t_{c}$ with $I=2$ but three times if $S$ happens to be in $V^{-} \cup V_{3}^{+}$when $t>t_{c}$ with $I=3$.

4.3. The class $I=4$. The critical curve $\overline{\mathcal{J}}$ has one local minimum and one maximum. We consider the case of constant rate of acceleration from an initial Mach number $M_{0}$, i. e., $M(\tau)=M_{0}+\tau^{2}$, with $F(\tau)=2 \tau(t-\tau)-M\left(M^{2}-1\right)$ and $F^{\prime \prime}=$ $-2-6 M\left(M+4 \tau^{2}\right)<0$.

If $M_{0} \leq 1$, the supersonic interval begins at $\tau=t_{*} \geq 0$ with $M_{*}=1, \sigma_{*}=0$ and $\sigma_{*}^{\prime}>0$. Using $\{8\}$ and $F^{\prime \prime}<0,(4.1)$ has only one root and the curve $\overline{\mathcal{J}}$ has only one maximum point. Thus the case for $M_{0} \leq 1$ belongs to the class $I=3$.

To create an example of the class $I=4$, we consider $M_{0}>1$ with $t_{*}=0$ and $t^{*}=t$. With $F(0)<0$ and $F(t)<0$, or $\sigma^{\prime}(0)<0 \sigma^{\prime}(t)<0,(4.1)$ can have two roots or none. It is clear that $M_{0}^{2}-1$ has to be sufficiently small so that $F(\tau)$ can be nonnegative in $(0, t)$, i. e., (4.1) has two roots. For a given $t$, there is an upper bound below which two distinct roots exist. When $M_{0}$ is equal to the upper bound $M_{c 0}$, these two roots coincide in one double root $\tau_{c}$ for $F\left(\tau_{c}\right)=F^{\prime}\left(\tau_{c}\right)=0$. This and the equation $M_{c}=M_{c 0}+\tau_{c}^{2}$, define $\tau_{c}, M_{c}$

$\dagger$ This problem can be identified with the one with the source field created at $\tilde{\tau}=0$ while $M=b$ by the time shift, $\tilde{\tau}=\tau-b$ and $\tilde{t}=t-b$ with velocity $\tilde{U}(\tilde{\tau})=U(\tau)$. 
and $\tau_{c 0}$ as functions of $t . \dagger$ For example when $t=2.25$, we have $M_{c}=1.382, \tau_{c}=0.3328$ and $M_{c 0}=1.275$.

If the initial Mach number $M_{0} \geq M_{c 0},(4.1)$ does not have two distinct roots and hence the critical curve $\overline{\mathcal{J}}$ has no local maximum. Hence this case belongs to the class of $I=2$ in $\S 4.1$.

For $1<M_{0}<M_{c 0},(4.1)$ has two distinct roots, the critical curve $\overline{\mathcal{J}}$ has a local maximum and a local minimum as shown in Fig. 6 and the image $\overline{\mathcal{J}}$ has two cusps forming a "butterfly" as shown in the insert in Fig.7. In those two figures, we have $M(\tau)=1.15+\tau^{2}$, $\tau \in[0,2.25]$, i. e., $M_{0}=1.15$ and $t=2.25$. Since $M_{0}<M_{c 0}$, (4.1) has two distinct roots $\tau_{1}=0.6093$ and $\tau_{2}=0.0918$. We have $N=2$ and $I=4$.

As shown in Fig. 6 , the critical curve $\overline{\mathcal{J}}$ begins with $\sigma(0)=1.1111$ at point $\bar{T}$ on the semicircle $\overline{\mathcal{B}}$, decreases to a minimum at point $\bar{K}_{2}$ along the segment $\overline{\mathcal{J}}_{3}$, increases to the maximum at point $\bar{K}_{1}$ along $\overline{\mathcal{J}}_{2}$ and then decreases to the origin $\bar{P}$ along $\overline{\mathcal{J}}_{1}$. By Rule 1 the critical curve $\overline{\mathcal{J}}$ partitions the domain of dependence $\overline{\mathcal{D}}$ into $\bar{V}^{ \pm}$. By Rule 2 and $\mathbf{3}$, these two domains, $\bar{V}^{ \pm}$, are partitioned respectively by the large circular arc $\overline{\mathcal{C}}_{1}^{+}$passing through $\bar{K}_{1}$ and the small circular arc $\overline{\mathcal{C}}_{2}^{-}$passing through $\bar{K}_{2}$ into $\bar{V}_{1}^{+}, \bar{V}_{3}^{+}$and $\bar{V}_{2}^{-}, \bar{V}_{4}^{-}$. Thus the domain of dependence $\overline{\mathcal{D}}$ is partitioned into four nonoverlapping domains.

Figure 7 shows the images of the critical curve, the semicircle when $\tau=0$ and the two circular arcs in the $\xi \sigma$ plane. Because the initial Mach number $M(0)$ is above 1 and below the threshold value $M_{c 0}$, the circular arc $\mathcal{C}_{2}^{-}$is approaching the semicircle $\mathcal{B}$ from the right hand side while the branches $\mathcal{J}_{2}$ and $\mathcal{J}_{3}$ and the two cusps $K_{1}$ and $K_{2}$ can be seen only in the insert with $20 \times$ enlargement. The domain $V_{4}^{-}$bounded by $\mathcal{B}, \mathcal{C}_{2}^{-} \mathcal{J}_{3}$ and the $\xi$-axis is disappearing. In addition to the $\xi$-axis, $V_{2}^{-}$is bounded by $\mathcal{J}_{2}, \mathcal{J}_{1}$ and $\mathcal{C}_{2}^{-}, V_{1}^{+}$ by $\mathcal{C}_{1}^{+}$and $\mathcal{J}_{1}$, and $V_{3}^{+}$by $\mathcal{B}, \mathcal{J}_{2}, \mathcal{J}_{3}$ and $\mathcal{C}_{1}^{+}$. The branch $\mathcal{J}_{3}$ begins at point $T$ tangent to the circle $\mathcal{B}$, goes downwards on the right hand side of $\mathcal{B}$ as the envelope of the circles of constant $\tau \in\left[0 . \tau_{2}\right.$ and ends at the cusp $K_{2}$. The branches $\mathcal{J}_{3}$ and $\mathcal{J}_{2}$ and the semicircle $\mathcal{B}$ form a curvilinear triangle which is the intersection of the four domains of integration in the $\xi \sigma$ plane. The intersection has the maximum multiplicity 4 .

If we increase $M(0)$ to the threshold value $M_{c 0}$, the two cusps $K_{1}$ and $K_{2}$ and the branch $\mathcal{J}_{2}$ disappear. Branch $\mathcal{J}_{1}$ joins smoothly with $\mathcal{J}_{3}$ to form a smooth critical curve $\mathcal{J}$ with no local maximum or cusp. The partitions by the arcs $\mathcal{C}_{1}^{+}$and $\mathcal{C}_{2}^{-}$are no longer needed. Thus we recover the class of $I=2$. If we decrease $M(0)$ to 1 , the minimum point $K_{2}$ moves down to the $\xi$-axis and leftward to $\mathcal{B}$ while the branch $\mathcal{J}_{3}$ and the domain $V_{4}^{-}$ disappear. Thus we recover the class of $I=3$.

Similar to the discussion in $\S 4.2$, for a given $M(0)=1.15$, the critical time of observation, when $M_{c 0}=M(0)$, is $t_{c}=1.074$. When $t \leq t_{c}$, an observer will receive signals from point $S$ at most twice in the class of $I=2$. When $t$ exceeds $t_{c}$, the observer can receive signals from the same point $S$ four times in the class of $I=4$.

$\dagger$ From $F^{\prime}\left(\tau_{c}\right)=0$ we get $\tau_{c}^{2}=\left(M_{c}^{2}-1\right) /\left(6 M_{c}\right)$ and from $F\left(\tau_{c}\right)=0, t^{2}=\left(M_{c}^{2}-\right.$ 1) $\left(3 M_{c}^{2}+1\right)^{2} /\left(6 M_{c}\right)$. The second equation defines $t>0$ for a $M_{c}>1$. The inverse transformation is also unique. Thus for a given $t>0$, the second equation yields a unique $M_{c}>1$ and then the first one, $\tau_{c}>0$. They in turn define the corresponding initial Mach number $M_{c 0}=\left(5 M_{c}^{2}+1\right) / 6 M_{c}>1$. 
4.4. $I=5$. The critical curve $\overline{\mathcal{J}}$ has one local minimum and two local maximum. It follows from $\{7\}$ and $\{8\}$ that $\bar{K}_{2}$ is the local minimum point and $\bar{K}_{1}$ and $\bar{K}_{3}$ are the two maximum points, with $\tau_{1}<\tau_{2}<\tau_{3}$ and that $\sigma^{\prime}\left(t_{*}\right) \geq 0$ and $M\left(t_{*}\right)=1$ for $t_{*} \geq 0$ or $M\left(t_{*}\right)>1$ for $t_{*}=0$.

We consider the case that the acceleration is a quadratic function of $\tau$,

$$
M^{\prime}(\tau)=c_{0}+2 c_{1} \tau+3 c_{2} \tau^{2}
$$

and hence $M$ is a cubic function,

$$
M(\tau)=\left[c_{0}+c_{1} \tau+c_{2} \tau^{2}\right] \tau,
$$

with $c_{0}+c_{1}+c_{2}=1$. We have chosen the time scale such that $M(0)=0$ and $M(1)=1$. As explained in $\S 4.2$, a nonzero initial value $M(0)$ can be converted to (4.4) by an appropriate time shift and change of coefficients $c_{0}$ and $c_{1}$.

We consider the case that the speed $U=M$ is accelerated to supersonic $M_{a}>1$ at $\tau=a>1$, followed by an interval of deceleration to $M_{b}$ at $\tau=b$ and then acceleration to $\tau=t$. To show the accelerating and decelerating intervals explicitly, we rewrite (4.3) as $†$

$$
M^{\prime}=c(\tau-a)(\tau-b) \quad \text { with } \quad b>a>1 \text { and } c=6 /[2-3(a+b)+6 a b] .
$$

To insure that $M>1$ during deceleration, we assume that $M(b)>1$.

Using (4.5), we find $F(1)>0, F(a)<0$ and $F^{\prime}(\tau)<0$ in the interval $(1, a)$, therefore, (4.1) has only one root in the interval. Since $F(b)<0, F(t)<0$ while $F^{\prime \prime}(\tau)<0$ in the interval $(b, t),(4.1)$ has either two roots or none in the interval $(b, t)$. Therefore, $\overline{\mathcal{J}}$ will have a maximum in the first acceleration interval, $(1, a)$ and a minimum and maximum or none in the second acceleration interval, $(b, t)$, in agreement with $\{8 a\}$. Whether there is a pair or not in the second interval, i. e., whether $I=5$ or 3 , depends on the values of $a, b$ and $t$. Let us consider the function $M(\tau)$ specified, i. e., $a$ and $b$ specified while $t$ varies, as we study the signal received at point $P$ from a point $S$. It is clear that when $1<t<b$, (4.1) has only one root, and $\overline{\mathcal{J}}$ has only a maximum. We have the class of $I=3$. There is critical time $t_{c}>b$ when there is a double root $\tau_{d}$ of $(4.1)$ in the interval $\left(b, t_{c}\right)$. By eliminating $\left(t_{c}-\tau_{d}\right)$ from $F\left(\tau_{d}\right)=0$ and $F^{\prime}\left(\tau_{d}\right)=0$, we get an equation for $\tau_{d}$,

$$
M^{\prime \prime}\left(\tau_{d}\right)\left[M^{2}\left(\tau_{d}\right)-1\right]-3 M\left(\tau_{d}\right)\left[M^{\prime}\left(\tau_{d}\right)\right]^{2}=0
$$

and then compute $t_{c}$ from (4.1). We have an example for the class of $I=5$, when $t>t_{c}$.

Figure 9 shows the domain $\overline{\mathcal{D}}$ and the critical curve $\overline{\mathcal{J}}$ for $a=1.7, b=3.05$ and $t=5$. Now $t_{c}=3.666<t$, we have an example for the class of $I=5$. The curve $\overline{\mathcal{J}}$ which partitions $\overline{\mathcal{D}}$ into $\bar{V}^{ \pm}$, has a maximum point $\bar{K}_{1}(0.880,0.642)$ at $\tau_{1}=3.911$, a minimum point $K_{2}(1.941,0.287)$ at $\tau_{2}=3.077>b$ and then a maximum point $\bar{K}_{3}(3.078,1.641)$ at $\tau_{3}=1.511$. The larger circular $\operatorname{arcs} \mathcal{C}_{1}^{+}$of $\tau_{1}$ and $\mathcal{C}_{3}^{+}$of $\tau_{3}$ partition the domain $\bar{V}^{+}$into

$\dagger$ Here we assume that the quadratic function (4.4) has two roots in the interval $(1, t)$. We can have examples of $I=5$ with $M^{\prime}=\left[(\tau+a)^{2}+b^{2}\right] /\left[(1+a)^{2}+b^{2}\right]>0$. See the report [6] 
three, $\bar{V}_{1}^{+}, \bar{V}_{3}^{+}$and $\bar{V}_{5}^{+}$. The smaller circular arc $\overline{\mathcal{C}}_{2}^{-}$partitions the domain $\bar{V}^{-}$into two, $\bar{V}_{2}^{-}$and $\bar{V}_{4}^{-}$.

The images of these five nonoverlapping subdomains of $\overline{\mathcal{D}}$ in the $\xi \sigma$ plane are defined one by one and the acoustic potential $\Phi(t, x, y, z)$ is given by (3.5) as the sum of integrals over the five images $V_{1}^{+}, V_{2}^{-}, V_{3}^{+}, V_{4}^{-}$and $V_{5}^{+}$. These five image subdomains are superimposed onto the $\xi \sigma$ plane as shown in Fig. 10a. Their union is the domain of dependence $\mathcal{D}$. The five subdomains $V_{i}^{ \pm}$can in turn be identified from Fig. 10a by tracing the images of the boundaries of $\bar{V}_{i}^{ \pm}$.

Fig. 10b shows the critical curve $\mathcal{J}$ enlarged five times in order to display the four branches and the curvilinear triangle bounded by the three branches $\mathcal{J}_{1}, \mathcal{J}_{2}$ and $\mathcal{J}_{3}$ which is the intersection of the five subdomains. The critical curve and the part of the semicircular $\mathcal{B}$ in the enlargement are shown in heavier lines than those for the three circular arcs $\mathcal{C}_{1}^{+}$, $\mathcal{C}_{2}^{-}$and $\mathcal{C}_{3}^{+}$because multiplicity does not change across these three arcs, according to Rule $\mathcal{C}$. The numerals in bold face indicate the multiplicities in a region $\mathcal{R}$ bounded by $\mathcal{B}, \mathcal{J}$ and/or the $\xi$ axis. We demonstrate the counting of multiplicities by applying Rules $\mathcal{B}$ and $\mathcal{J}$ instead of identifying $\mathcal{R}$ as the intersection of subdomains $V_{i}^{ \pm}$and counting the number of subdomains in the intersection. For example, we show by counting that in the curvilinear triangle the multiplicity is 5 .

Consider the change of multiplicities from left to right along a horizontal line $\mathcal{L}$ of constant $\sigma_{c}$ below the cusp $K_{1}$ and above $K_{2}$, i. e., $\sigma_{2}<\sigma_{c}<\sigma_{1}$. A point on $\mathcal{L}$ lying to the left of the semicircle $\mathcal{B}$ is outside of $\mathcal{D}$ and has multiplicity $m=0$. When $\mathcal{L}$ crosses $\mathcal{B}$ from left to right and enters $V_{5}^{+}$, the multiplicity $m$ changes to 1 according to Rule $\mathcal{B}$. When $\mathcal{L}$ crosses the branch $\mathcal{J}_{4}, m=3$ according to Rule $\mathcal{J}$. When $\mathcal{L}$ crosses $\mathcal{J}_{2}$ and enters the curvilinear triangle, $m=5$. When $\mathcal{L}$ leaves the triangle and crosses $\mathcal{J}_{1}, \mathcal{J}_{3}$ (or $\mathcal{J}_{3}, \mathcal{J}_{1}$ ), and then $\mathcal{B}, m=3,1$ and then 0 . If it crosses $\mathcal{J}_{3}, \mathcal{B}$ and then $\mathcal{J}_{1}, m=3,2$ and then 0 . If the horizontal line is below $K_{2}, m$ changes from $0,1,3,2$ and then 0 . If the line is between $K_{1}$ and $K_{3}, m=0,1,3,1$ and then 0 . If the line is above $K_{3}$ but below $\sigma=t$, $m=0,1$ and then 0 .

It is clear that we can find examples which require an even greater number of partitions, but we have given plenty of examples to demonstrate the rules of partitions and counting of multiplicities. We note that although the velocity $U$ or Mach number $M$ in the examples are polynomials of $\tau$, the rules are applicable when $M$ is not a polynomial. This is demonstrated in the following example.

4.5. The speed $U(\tau)$ is periodic We consider $U=M=u_{0}-(c / a) \cos (a \tau+b)$ and $t=\pi / a$. Fig.11 shows the domain of dependence $\overline{\mathcal{D}}$ and the critical curve $\overline{\mathcal{J}}$ with $a=$ $1, b=0$ and $c=0.8 a$ and $u_{0}=M_{0}=2$ and $t=\pi$. The speed is supersonir in the interval $[0, t]$. The critical curve begins on the circle $\overline{\mathcal{B}}$ and ends at the origin. Thrre is one local minimum $\bar{K}_{2}$ and one maximum $\bar{K}_{1}$ at $\tau$ equal to 0.28194 and 0.67991 . $\|i\|_{1} .{ }^{\prime}=2$ and $I=4$, the partition of $\overline{\mathcal{D}}$ into four subdomains is similar to that in Fig. $G$. Figure 12a shows the image of Fig. 11 in the $\xi \sigma$ plane. Figure $12 \mathrm{~b}$ shows the $45 \times$ enlargement of the region containing the cusps $K_{1}$ and $K_{2}$ and the area bounded by the three branches, $\mathcal{J}_{1}$, $\mathcal{J}_{2}$, and $\mathcal{J}_{3}$, only in which the multiplicity is the maximum, 4. Again note the similarity of Fig. 12 to Fig. 7.

In the following subsections we shall present examples with more than one supersonic 
interval in $[0, t]$. Let there be two intervals $\left(\iota_{2}, \iota_{1}\right)$ and $\left(\iota_{4}, \iota_{3}\right)$ with $0 \leq \iota_{4}<\iota_{3} \leq \iota_{2}<$ $\iota_{1} \leq t$. The speed is subsonic in $\left(\iota_{3}, \iota_{2}\right)$ and sonic at the end points. From $\{7\}$, we see that there is at least one maximum on the critical curve in the first interval $\left(\iota_{2}, \iota_{1}\right)$. In the second interval, the critical curve may not have an extremum, if $\iota_{4}=0$ and the speed is decelerating from supersonic speed $M(0)$ to sonic speed. Since $N \geq 1$ and $I=1+s+N \geq 4$ for $s \geq 2$, we deal first with the class $I=4$.

4.6. Class $I=4, s=2$, two critical curves having only one local maximum. As a simple example. we consider a linearly accelerating motion in the interval $[0, t]$,

$$
M^{\prime}(\tau)=\tau-b, \quad \text { with } \quad b \in(0, t)
$$

The velocity function is

$$
M(\tau)=M_{0}-b \tau+\tau^{2} / 2, \text { with } M_{0}>1 \text { and } M(t)>1 .
$$

We choose $M_{0}, b$ and $t$ such that the minimum $M_{b}=M(b)=M_{0}-b^{2} / 2$ is subsonic i. e., $1>M_{b}>0$. The speed becomes sonic at $\iota_{2}$ and $\iota_{3}$, where

$$
\iota_{2}=b+\sqrt{b^{2}-2 c} \text { and } \iota_{3}=b-\sqrt{b^{2}-2 c},
$$

with $c=M_{0}-1$. Here we have two supersonic intervals $\left(\iota_{2}, t\right]$ and $\left[0, \iota_{3}\right)$. Only the critical curve in the first interval has a maximum at point $\bar{K}_{1}$ at the retarded time $\tau_{1} \in\left(\iota_{2}, t\right)$. As shown in Fig. 13, the domain of dependence $\overline{\mathcal{D}}$ is partitioned into four nonoverlapping regions $\bar{V}_{1}^{+}, \bar{V}_{2}^{-}, \bar{V}_{3}^{+}$and $\bar{V}_{4}^{-}$. The region $\bar{V}_{2}^{-}$is bounded by the $\bar{\xi}$ axis and the two segments of the critical curve, $\overline{\mathcal{J}}_{1}^{1}$ as $\tau$ decreases from $t$ to $\tau_{1}$ and $\overline{\mathcal{J}}_{2}^{1}$ from $\tau_{1}$ to $\iota_{2}$. The region $\bar{V}_{4}^{-}$is bounded by the $\bar{\xi}$ axis, the small circular arc $\bar{B}^{-}$and the critical curve $\overline{\mathcal{J}}^{2}$ from $\iota_{3}$ to 0 . The region, where $J>0$, is partitioned by the large circular arc $\overline{\mathcal{C}}_{1}^{+}$of constant retarded time $\tau_{1}$ into $\bar{V}_{1}^{+}$and $\bar{V}_{3}^{+}$. The segments on the $\bar{\xi}$ axis bounding $\bar{V}_{2}^{-}$and $\bar{V}_{4}^{-}$, where $J<0$, are $\bar{\xi}_{1} \bar{\xi}_{2}$ and $\bar{\xi}_{3} \bar{\xi}_{4}$. With $\bar{\xi}_{j}=C\left(t-\iota_{j}\right)$, we have $\bar{\xi}_{j}>\bar{\xi}_{j-1}$. Here we have $\iota_{1}=t, \bar{\xi}_{1}=0$ and $\iota_{4}=0, \bar{\xi}_{4}=C t$.

If the images of $\bar{V}_{2}^{-}$and $\bar{V}_{4}^{-}$do not intersect, i. e., $V_{2}^{-} \cap V_{4}^{-}=\emptyset$, the maximum multiplcity of $D$ can only be $3<I=4$. This requires that the image segments $\xi_{1} \xi_{2}$ and $\xi_{3} \xi_{4}$ do not overlap. Noting the sign of $J$, we have $\xi_{2}>\xi_{1}$ and $\xi_{4}>\xi_{3}$ while $\xi_{2}<\xi_{3}$. For the two segments to be nonoverlapping, it is necessary that $\xi_{1}<\xi_{4}$, i. e.,

$$
\bar{\xi}_{4}-\bar{\xi}_{1}>\int_{\iota_{4}}^{\iota_{1}} M(\tau) d \tau, \text { or } \quad<M>=\frac{\int_{\iota_{4}}^{\iota_{1}} M(\tau) d \tau}{\iota_{1}-\iota_{4}}<1 .
$$

This says that the average Mach number $\langle M\rangle$ or speed $\langle U\rangle$ in the interval $\left[\iota_{4}, \iota_{1}\right]$, has to be subsonic. This statement is valid for any $M(\tau)$ having two supersonic intervals and an intermediate subsonic interval in $\left[\iota_{4}, \iota_{1}\right]$.

In Fig. 13, we have $b=1.0, M_{0}=1.18, X(0)=0$ and $t=2.4$. The speed is sonic at $\iota_{2}=1.8 \iota_{3}=0.2, M_{b}=0.68$ and $\langle M\rangle=0.94<1$. The images of the four subdomains in Fig. 13 are shown in Fig. 14a. A $3 \times$ enlargement of the region containing the critical curves is shown in Fig.14b where the bold faced numerals denote the multiplicity. In this 
case the subdomains $V_{2}^{-}$and $V_{4}^{-}$do not overlap and the maximum multiplicity is equal to $3<I=4$.

Figures 15 and $16 \mathrm{a}$ and $\mathrm{b}$ show a counter example of Figs. 13 and $14 \mathrm{a}$ and $\mathrm{b}$ with the same $t=2.4$, and smaller $b=0.8$. The speed is sonic at $\iota_{2}=1.33$ and $\iota_{3}=0.27$, with $M_{b}=0.70$. There is a longer second supersonic interval and the average Mach number $<M>=1.18$ is supersonic. As shown in Fig. 16, the subdomains $V_{2}^{-}$and $V_{4}^{-}$do overlap and the maximum multiplicity is equal to $I=4$.

4.7. More than two critical curves, each having only one local maximum We consider an example with periodic velocity variation about a mean subsonic speed. Then there are three subsonic and three supersonic intervals. We choose $X(0)=0, t=5.0$ and $M^{\prime}(\tau)=0.5 \pi \sin (\pi \tau)$ and $M(\tau)=0.75-0.5 \cos (\pi \tau)$.

As shown in Fig. 17, the domain of dependence $\overline{\mathcal{D}}$ is partitioned into seven nonoverlapping regions by the three nonintersecting critical curves $\overline{\mathcal{J}}^{i}, i=1,2,3$ and the large circular arcs $\overline{\mathcal{C}}^{i}$ passing through the maxima $\bar{K}^{i}$. Therefore $I=3+3+1=7$. The images of the seven subdomains in Fig. 17 are shown in Fig. 18a. A $2 \times$ enlargement of the region containing the three critical curves is shown in Fig.18b where the bold faced numerals denote the multiplicity. In this case the maximum multiplicity is equal to $3<I=7$.

\section{Conclusion}

In the interaction of an acoustic field with a moving airframe we encounter a canonical initial value problem for an acoustic field induced by an unsteady source distribution, $q(t, \mathbf{x})$ with $q \equiv 0$ for $t \leq 0$, in a medium moving with a uniform unsteady velocity $U(t) \hat{\imath}$ in the coordinate system $\mathbf{x}$ fixed on the airframe. Signals issued from a source point $S$ in the domain of dependence $\mathcal{D}$ of an observation point $P$ at time $t$ may arrive at point $P$ more than once corresponding to different retarded times, $\tau$ in the interval $[0, t]$. The number of arrivals is called the multiplicity of the point $S$. The multiplicity equals 1 if the velocity $U$ remains subsonic and can be greater when $U$ becomes supersonic. For an unsteady uniform flow $U(t) \hat{\imath}$, the acoustic potential $\Phi(t, x, y, z)$ is governed by the convective wave equation with variable coefficients, the velocity $U(t)$ and the accelerati on $\dot{U}(t)$. The solution $\Phi(t, \mathbf{x})$ is given indirectly via the solution of the corresponding acoustic field $\bar{\Phi}$ in the coordinate system $\overline{\mathbf{x}}$ with the medium at rest. The solution (1.16) induced by the source distribution $q(t, \mathbf{x})$ is

$$
\begin{aligned}
\Phi(t, x, y, z) & =-\frac{1}{4 \pi} \iiint_{\mathcal{D}} d \xi d \eta d \zeta \frac{q(\tau, x+\xi, y+\eta, z+\zeta)}{|R-M \bar{\xi}|} \\
& =-\frac{1}{4 \pi} \sum_{i=1}^{I} \iiint_{V_{i}} d \xi d \eta d \zeta \frac{q\left(\tau_{i}, x+\xi, y+\eta, z+\zeta\right)}{\left|R_{i}-M_{i} \bar{\xi}_{i}\right|}
\end{aligned}
$$

To evaluate the integral over the domain of dependence $\mathcal{D}$ in (5.1a) we need to relate $\tau$, $R$ and $\bar{\xi}$ to $t$ and the coordinates of the source point $S(x+\xi, y+\eta, z+\zeta)$. The implicit relationships are:

$$
\bar{\xi}=\xi+\int_{\tau}^{t} U\left(t^{\prime}\right) d t^{\prime} \quad \text { and } \quad R^{2}=C(t-\tau)^{2}=\bar{\xi}^{2}+\eta^{2}+\zeta^{2}
$$


They yield multiple retarded times $\tau_{i}$ in $[0, t]$ and the corresponding $R_{i}$ and $\bar{\xi}_{i}$. We need to find the roots of (5.2) and identify the domain $V_{i}$ in the $\xi, \eta, \zeta$ space for each $\tau_{i}$. Then $\Phi$ is written as the sum of integrals over the smallest number $I$ of domains $V_{i}$ in (5.1b). Here $I$ denotes the maximum multiplicity of $\mathcal{D}$. It is the purpose of this paper to formulate the rules identifying these dom ains $V_{i}$ for a medium moving at an unsteady speed, $U(t)$. First we study the Jacobian of the transformation $\mathbf{x}$ to $\overline{\mathbf{x}}$ in $\S \mathbf{2}$ and then use these studies to formulate in $\S 3$ the rules for the partition of the domain of dependence, $\overline{\mathcal{D}}$ in the $\overline{\mathbf{x}}$ space, into nonoverlapping subdomains $\bar{V}_{j}$ for an unsteady velocity, $U(\tau), \tau \in[0, t]$. We show that the partition has the required property that the value of $\tau$ and hence $R$ and $\bar{\xi}$ defined by (5.2) for a point $(\xi, \eta, \zeta)$ in an image $V_{j}$ is uniquely defined. Thus the domain of integration in $(5.1 \mathrm{~b})$ is the sum of those images $V_{j}$ 's. Since those images may overlap in the $\xi, \eta, \zeta$ space, signals from a point $S$ in the space lying in $m$ of those images will reach point $P \quad m$-times. Hence $m$ is th e multiplicity of point $S$. The rules for counting the multiplicity of a point in $D$ are also stated in $\S 3$. The analyses in $\S 2$ and $\S 3$ justify the assertions made in [2] for the case of a constantly accelerating speed. Additional examples with unsteady velocities, accelerating and/or decelerating at different ranges of Mach numbers are presented in $\S 4$ to demonstrate the applicatio $n$ of the rules of partition of $\overline{\mathcal{D}}$ and to show the singularities of $\mathcal{J}$ in the $\xi, \eta, \zeta$ space.

Appendix A. Domain of dependence and the region of influence in a constant supersonic flow We study the domain of dependence $\mathcal{D}$ of an observation point $P$ at an instant $t>0$ for an acoustic field created in the interval $[0, t]$ and the region of influence of a source point $S$ during the interval $[\tau, t]$ where $\tau$ denotes the retarded time. Consider the meridian $\xi \sigma$ plane in the coordinate system $\xi, \eta, \zeta$ containing points $P$ and $S$. The $\xi$-axis is in the direction of the flow, $\hat{\imath}$. Point $P$ is the origin and $\mathcal{D}$ is bounded by the $\xi$ axis, the backward Mach line from $P$ and the large circular arc of radius $R_{0}=C t$ with $\tau=0$ and centered at $G$ on the $\xi$-axis with $\xi=-M R_{0}$. Note that the region of influence of a source point $S$ for the interval $[0, t]$ is the mirror image of the the domain of dependence of $P$ with respect to the $\sigma$ axis and point $S$ at the origin. Fig. $19 \mathrm{a}$ and b show the two domains of integration $V^{+}$and $V^{-}$for $M=\sqrt{2}$ in a meridian plane.

The backward Mach line from $P$ is the envelope of the semi-circles $\mathcal{C}$ of constant $\tau \in[0, t]$ with radii $R=C(t-\tau)$. The Mach line divides a semi-circle into two arcs, the larger arc $\mathcal{C}^{+}$is convex while the smaller arc $\mathcal{C}^{-}$is concave from the Mach cone. The domain $V^{+}$coincides with $\mathcal{D}$ and is covered by the family of convex circular arcs $\mathcal{C}^{+}$. The domain $V^{-}$is covered by the family of concave circular arcs, $\mathcal{C}^{-}$with $V^{+} \notin V^{-}$equal to the semi-circular disc of radius $C t$ centered at $G$. Consider a source point $S$ in $V^{-} \in V^{+}$. Point $S$ lies on the intersection of two circular arcs in $V^{ \pm}$, the convex circular arc $\mathcal{C}^{+}$ of radius $R^{+}$centered at $G^{+}$with $\xi=-M R^{+}$in $V^{+}$and the concave circular arc $\mathcal{C}^{-}$of radius $R^{-}$centered at $G^{-}$with $\xi=-M R^{-}$in $V^{-}$. Note that these two arcs are associated with different semi-circles or different retarded times $\tau^{ \pm}$, with $\tau^{+}>\tau^{-}$. In Fig. 19a and $19 \mathrm{~b}$, we choose $\tau^{+}=0.75 t$ and $\tau^{-}=0.25 t$. The signal created at the retarded time $\tau^{-}$at point $S$ in Fig. 19b propagates at time $t$ onto a sphere of radius $R^{-}$while its center travels parallel to $\hat{\imath}$ to $S^{-}$with $\left|S S^{-}\right|=M R^{-}=\left|G^{-} P\right|$. The region of influence of point $S$ with retarded time $\tau^{-}$(in the meridian plane) is bounded by the two forward Mach lines from $S$ and the circular arc of radius $R^{-}$centered at $S^{-}$. Since points $S, S^{-}, P$ and $G^{-}$are the 
vertices of a parallelgram, point $P$ lies on the small circular arc. The signal initiated at time $\tau^{-}$from point $S$ reaches point $P$ at time $t$. Likewise we see in Fig. 19a how the signal from $S$ at the retarded time $\tau^{+}$reaches $P$ at time $t$. Similarly, we can see for the case of an unsteady moving medium the connection between the region of influence of point $S$ in the interval $[\tau, t]$ and the propagation of the signal from $S$ initiated at $\tau$ reaching point $P$ at time $t$.

\section{Appendix B. Transformation of a Neighborhood of $\overline{\mathcal{J}}$ in the $\bar{\xi} \sigma$ plane onto the $\xi \sigma$ plane and the Inverse Transformation}

We study the transformation of a point $\bar{S}\left(\bar{\xi}, \sigma_{c}\right)$ in the neighborhood of the critical curve $\overline{\mathcal{J}}$ onto a point $S\left(\xi, \sigma_{c}\right)$ near $\mathcal{J}$ and its inverse transformation. We add the subscript $c$ to $\sigma$ to shown that $\sigma$ remains unchanged in the transformation.

First we consider the point $\bar{S}$ near a branch of $\overline{\mathcal{J}}$ but not too close to the extremum point $\bar{K}_{1}$. Using the same ordinate $\sigma_{c}$ for the neighboring point $\bar{S}_{c}\left(\bar{\xi}_{c}, \sigma_{c}\right)$ on $\overline{\mathcal{J}}_{1}$, we use (1.9) and the equation for $\sigma$ in (2.2) or (2.5) to find $\tau_{c}$ or $R_{c}$ first and then $\bar{\xi}_{c}$ from (2.2), and $\xi_{c}$ from (2.5), for the corresponding point $S_{c}\left(\xi_{c}, \sigma_{c}\right)$ on $\mathcal{J}$. Here the subscript $c$ denotes the value at $\tau_{c}$.

Note that we are considering,

$$
\left|\bar{\xi}-\xi_{c}\right| \ll 1 \text { and hence }\left|\tau-\tau_{c}\right| \ll 1 \text { while } \sigma_{c}^{\prime} \neq 0
$$

Here $\tau$ denotes the retarded time corresponding to point $\bar{S}$ by (1.9),

$$
\tau=t-R / C \text { and } R=\sqrt{\bar{\xi}^{2}+\sigma_{c}^{2}} .
$$

Using (B.1) we simplify the transformation (1.12) from $\bar{\xi}$ to $\xi$. Using (1.12) and expressing $M$ by the Taylor series in $\left(\tau-\tau_{c}\right)$, we have

$$
\xi-\xi_{c}=\bar{\xi}-\bar{\xi}_{c}+\int_{\tau_{c}}^{\tau} C M(\tilde{\tau}) d \tilde{\tau}=\bar{\xi}-\bar{\xi}_{c}+C M_{c}\left(\tau-\tau_{c}\right)+\frac{C}{2} M_{c}^{\prime}\left(\tau-\tau_{c}\right)^{2}+O\left(\left(\tau-\tau_{c}\right)^{3}\right),
$$

Now we express $C\left(\tau-\tau_{c}\right)$ in power series of $\delta_{c}=\left(\bar{\xi}-\bar{\xi}_{c}\right)$,

$$
\begin{aligned}
C\left(\tau-\tau_{c}\right) & =-\left(R-R_{c}\right)=-\left(\partial_{\bar{\xi}} R\right)_{c} \delta_{c}-\frac{1}{2}\left(\partial_{\bar{\xi} \bar{\xi}}^{2} R\right)_{c} \delta_{c}^{2}+O\left(\delta_{c}^{3}\right) \\
& =-\frac{1}{M_{c}} \delta_{c}-\frac{\sigma_{c}^{2}}{2 R^{3}} \delta_{c}^{2}+O\left(\delta_{c}^{3}\right) .
\end{aligned}
$$

Using (2.3a) and (B.4) in (B.3), we get

$$
\begin{aligned}
\xi-\xi_{c} & =\frac{M_{c}^{\prime} R_{c}-C M_{c}\left(M_{c}^{2}-1\right)}{2 C M_{c}^{2} R_{c}} \delta_{c}^{2}+O\left(\delta_{c}^{3}\right) \\
& =\frac{\sqrt{M_{c}^{2}-1} \sigma_{c}^{\prime}}{2 C R_{c}}\left(\bar{\xi}-\bar{\xi}_{c}\right)^{2}+O\left(\delta_{c}^{3}\right),
\end{aligned}
$$


which in turn defines the inverse transformation,

$$
\bar{\xi}=\bar{\xi}_{c} \pm \sqrt{2 C R_{c}}\left(M_{c}^{2}-1\right)^{-1 / 4} \sqrt{\left(\xi-\xi_{c}\right) / \sigma_{c}^{\prime}} .
$$

This implies that a point $S$ in the neighborhood of a branch $\mathcal{J}_{1}$ (B.1), on the side with

$$
\sigma_{c}^{\prime}\left(\xi-\xi_{c}\right)>0
$$

is mapped to two points on both sides of $\overline{\mathcal{J}}$. For a point $S$ on the other side of $\mathcal{J}$, with $\left(\xi-\xi_{c}\right) \sigma_{c}^{\prime}<0$, (B.6) says only that there is no inverse solution $\bar{S}$ in the neighborhood of point $\bar{S}_{c}$. Because of (B.1), (B.6) does not include inverse solution(s) of (1.12) which are away from the branch $\overline{\mathcal{J}}_{1}$. From (2.6), $\sigma_{c}^{\prime}$ and $\xi_{c}^{\prime}$ are of oppsite sign and (B.6) implies

(i) Along a branch of $\overline{\mathcal{J}}$ where $\sigma_{c}$ increases (decreases) with increasing $\tau_{c}$, a point $\bar{S}$ on either side of $\overline{\mathcal{J}}$ is mapped to a point $S$ on the right (left) hand side of $\mathcal{J}$ with $\xi-\xi_{c}$ positive (negative) while $\xi_{c}$ decreases (increases) along $\mathcal{J}$. The multiplicity of the point $S$ is at least 2 .

Now consider the case that the point $\bar{S}(\bar{\xi}, \sigma)$ is close to an extremum point on $\overline{\mathcal{J}}$, say at point $\bar{K}_{1}\left(\bar{\xi}_{1}, \sigma_{1}\right)$ where $\sigma_{1}^{\prime}=0$. Here we use subscript 1 to denote the value at $\tau_{1}$. The distance between $\bar{S}$ and $\bar{K}_{1}$ is small, i. e., $\left|\bar{S} \bar{K}_{1}\right|=\sqrt{\left(\bar{\xi}-\bar{\xi}_{1}\right)^{2}+\left(\sigma-\sigma_{1}\right)^{2}} \ll 1$, and hence

$$
C\left|\tau-\tau_{1}\right|=\left|R-R_{1}\right| \leq\left|\bar{S} \bar{K}_{1}\right| \ll 1 .
$$

Because of $\sigma_{1}^{\prime}=0$, we need to include at least the third order terms in the representation of the transformation (B.3) by a power series of $\tau-\tau_{1}$ or rather $R-R_{1}$,

$$
\xi-\xi_{1}=\bar{\xi}-\bar{\xi}_{1}-M_{1}\left(R-R_{1}\right)+\frac{1}{2 C} M_{1}^{\prime}\left(R-R_{1}\right)^{2}-\frac{1}{6 C^{2}} M_{1}^{\prime \prime}\left(R-R_{1}\right)^{3}+O\left(\left(R-R_{1}\right)^{4}\right) .
$$

With $\bar{\xi}=\sqrt{R^{2}-\sigma^{2}}$, we expand $\bar{\xi}-\bar{\xi}_{1}$ in a double power series of $R-R_{1}$ and $\sigma-\sigma_{1}$. Using (2.4b), we note

$$
\left|\sigma-\sigma_{1}\right| \leq\left|\sigma_{c}-\sigma_{1}\right|=O\left(\left(\xi_{c}-\xi_{1}\right)^{2}\right),
$$

while $\left|R-R_{1}\right|$ can be $O\left(\left|\xi_{c}-\xi_{1}\right|\right)$. We keep $O\left(\left|R-R_{1}\right|^{3}\right)$ terms but not fourth order terms, e. g., $\left(\sigma-\sigma_{1}\right)^{2}$ and $\left(R-R_{1}\right)^{2}\left(\sigma-\sigma_{1}\right)$, and get

$$
\begin{aligned}
\bar{\xi}-\bar{\xi}_{1} & =\left(\frac{\partial \bar{\xi}}{\partial R}\right)_{1}\left(R-R_{1}\right)+\frac{1}{2}\left(\frac{\partial^{2} \bar{\xi}}{\partial R^{2}}\right)_{1}\left(R-R_{1}\right)^{2}+\frac{1}{6}\left(\frac{\partial^{3} \bar{\xi}}{\partial R^{3}}\right)_{1}\left(R-R_{1}\right)^{3} \\
& +\left(\frac{\partial \bar{\xi}}{\partial \sigma}\right)_{1}\left(\sigma-\sigma_{1}\right)+\left(\frac{\partial^{2} \bar{\xi}}{\partial R \partial \sigma}\right)_{1}\left(R-R_{1}\right)\left(\sigma-\sigma_{1}\right)+O\left(\left(R-R_{1}\right)^{4}\right) .
\end{aligned}
$$

With the partial derivatives at $\tau_{1}$,

$$
\frac{\partial \bar{\xi}}{\partial R}=\frac{R_{1}}{\bar{\xi}_{1}}=M_{1}, \quad \frac{\partial^{2} \bar{\xi}}{\partial R^{2}}=-\frac{\sigma_{1}^{2}}{\bar{\xi}_{1}^{3}}=-\frac{M_{1}\left(M_{1}^{2}-1\right)}{R_{1}},
$$




$$
\begin{gathered}
\frac{\partial^{3} \bar{\xi}}{\partial R^{3}}=\frac{3 \sigma_{1}^{2} R_{1}}{\bar{\xi}_{1}^{5}}=\frac{3 M_{1}^{3}\left(M_{1}^{2}-1\right)}{R_{1}^{2}} \\
\frac{\partial \bar{\xi}}{\partial \sigma}=-\frac{\sigma_{1}}{\bar{\xi}_{1}}=-\sqrt{M_{1}^{2}-1} \text { and } \frac{\partial^{2} \bar{\xi}}{\partial \sigma \partial R}=\frac{\sigma_{1} R_{1}}{\bar{\xi}_{1}^{3}}=\frac{M_{1}^{2} \sqrt{M_{1}^{2}-1}}{R_{1}}
\end{gathered}
$$

(B.11) and (B.9) yield

$$
\begin{aligned}
\xi-\xi_{1}= & -\sqrt{M_{1}^{2}-1}\left(\sigma-\sigma_{1}\right)+\frac{M_{1}^{2} \sqrt{M_{1}^{2}-1}}{R_{1}}\left(\sigma-\sigma_{1}\right)\left(R-R_{1}\right) \\
& -\frac{\sigma_{1}^{\prime \prime} M_{1}^{2} \sqrt{M_{1}^{2}-1}}{6 C^{2} R_{1}}\left(R-R_{1}\right)^{3}+O\left(\left|\xi_{c}-\xi_{1}\right|^{4}\right) .
\end{aligned}
$$

Since $\sigma, \sigma_{1}$ and $R_{1}$ are known, (B.12) becomes a cubic equation for $\left(R-R_{1}\right)$ or $R$,

$$
\left(R-R_{1}\right)^{3}+p\left(R-R_{1}\right)+q=0,
$$

where

$$
p=\frac{-6 C^{2}\left(\sigma-\sigma_{1}\right)}{\sigma_{1}^{\prime \prime}}, \quad q=\frac{6 C^{2} R_{1} H}{\sigma_{1}^{\prime \prime} M_{1}^{2} \sqrt{M_{1}^{2}-1}} \quad \text { and } \quad H=\left(\xi-\xi_{1}\right)+\left(\sigma-\sigma_{1}\right) \sqrt{M_{1}^{2}-1}
$$

Here $H$ denotes the horizontal distance from point $(\xi, \sigma)$ to the tangent line of $\mathcal{J}$ at the cusp point $K_{1}$. The character of the roots of (B.13) depends on the discriminant of the cubic, $\Omega=q^{2} / 4+p^{3} / 27$. If $\Omega<0$, (B.13) has three distinct real roots, $\Omega=0$, three real roots with two equal and $\Omega>0$, only one real root.

In order to have three real roots it is necessary for $p<0$ that

$$
\left(\sigma-\sigma_{1}\right) \sigma_{1}^{\prime \prime}>0 \text {. }
$$

This says that

(ii) For the cubic (B.13) to have three real roots, it is necessary that $\sigma$ should be below the local maximum $\sigma_{1}$ on $\mathcal{J}$ or above the local minimum.

When $\Omega \geq 0$, we have

$$
H^{2} \leq \frac{8 C^{2} M_{1}^{4}\left(M_{1}^{2}-1\right)}{9 \sigma_{1}^{\prime \prime} R_{1}^{2}}\left(\sigma-\sigma_{1}\right)^{3} .
$$

With the equality sign, (B.15) becomes the equation describing the two branches of $\mathcal{J}$ near the cusp $S_{1}$. For a point $S(\xi, \sigma)$ in the interior bounded by these two branches, we have $\Omega>0$, and hence there are three distinct real roots for $\bar{\xi}$ or $\tau$. In the exterior there is only one real root. Thus using (B.14) and (B.15) we conclude that

(iii) For a cusp with a local maximum $\sigma_{1}$, a point $S$ in the interior bounded by these two branches has image point $\bar{S}$ inside the domain $\overline{\mathcal{D}}^{+}$bounded by $\overline{\mathcal{J}}$ and two image points in $\overline{\mathcal{D}}^{-}$lying to the right and left of the local maximum point $\bar{S}_{1}$. For a point 
$S$ exterior to the region bounded by the two branches, there is only one image point $\bar{S}$ in $\overline{\mathcal{D}}^{-}$.

(iv) For a cusp with a local minimum $\sigma_{2}$, a point $S$ in the interior bounded by the two branches has one image point $\bar{S}$ in $\overline{\mathcal{D}}^{-}$and two image points in $\overline{\mathcal{D}}^{+}$lying to the right and left of the local minimum point $\bar{S}_{2}$. For a point $S$ exterior to the region bounded by the two branches, there is only one image point $\bar{S}$ in $\overline{\mathcal{D}}^{+}$.

Again we note that the above analysis is local and does not account for the roots of (1.12) not in the neighborhood of an extremum point, if any. Thus we say

(v) The multiplicity of a point $S(\xi, \sigma)$ near a cusp and in the interior bounded by the two branches is at least 3 .

\section{References}

[1] L. Maestrello, A. Frendi and D. E. Brown, Nonlinear vibration and radiation from a panel with transition to chaos induced by acoustic waves, AIAA Journal 30, (1992) 2632-2638.

[2] L. Ting, On-Surface conditions for Structural Acoustic Interactions in Moving Media, presented at the Workshop on "Perturbation Methods in Physical Mathematics", Rensselaer Polytechnic Institute, Troy, NY June 23-26, 1993, to appear in SIAM J. Appl. Math., 55, (1995).

[3] P. M. Morse and K. U. Ungard, Theoretical Acoustics, McGraw-Hill, New York, (1968).

[4] J. W. Bruce and P. J. Giblin, Curves and Singularities, Cambridge University Press, 2nd Ed., (1992).

[5] T. Poston and I. Stewart, Catastrophe Theory and its Applications, Pitman, San Francisco, (1978). 


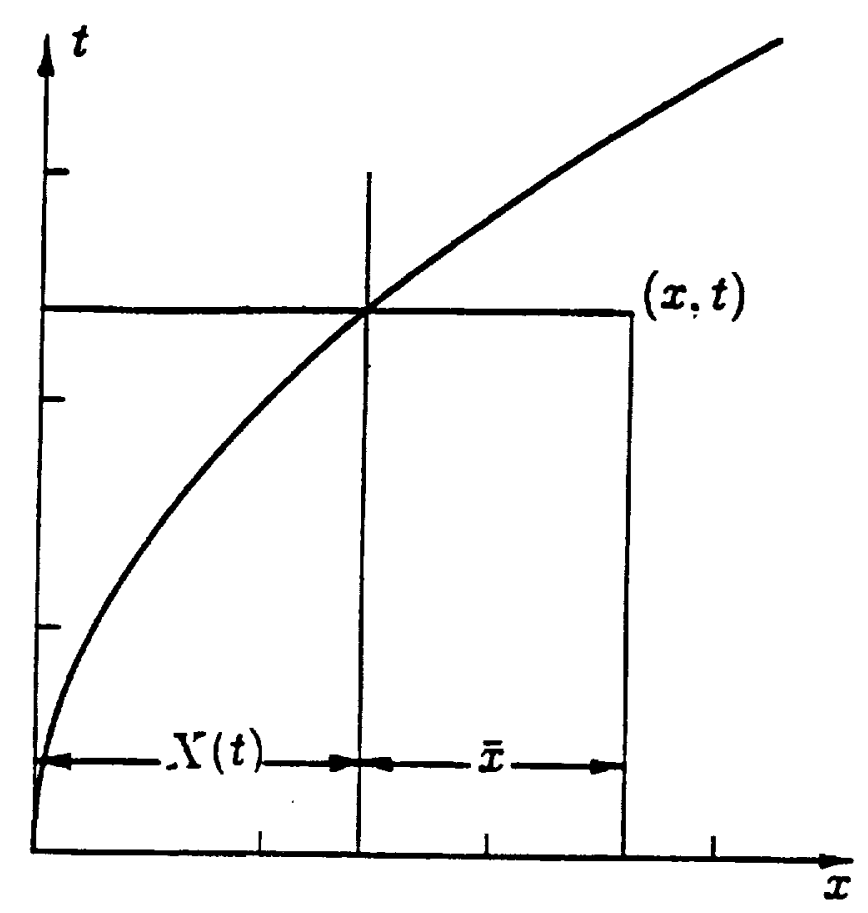

Fig. 1 The coordinate system $(\bar{x}, y, z)$ with the medium at rest and the system $(x, y, z)$, with $x=\bar{x}+X(t)$.

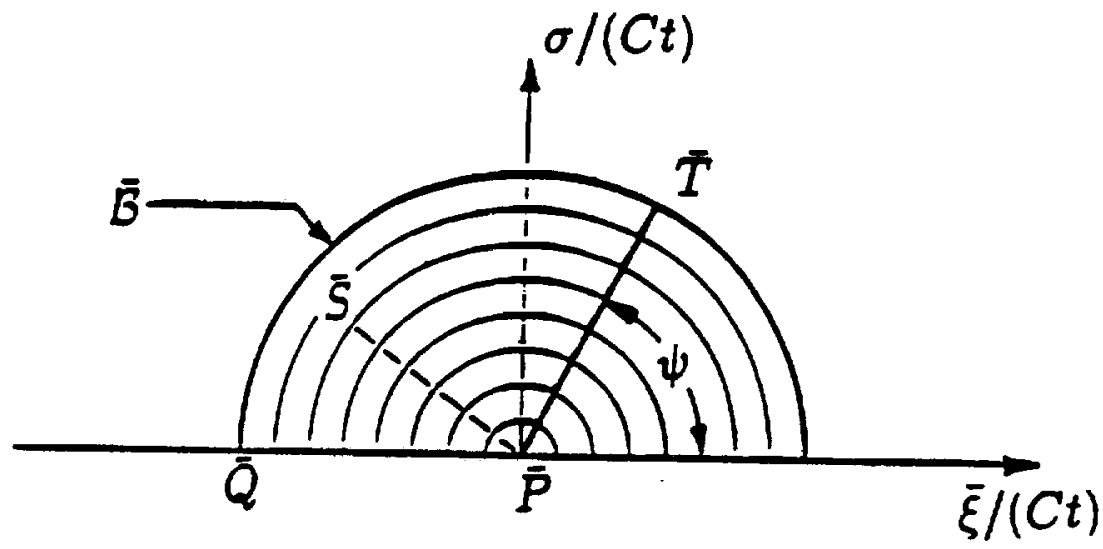

Fig. 2 The domain of dependence $\overline{\mathcal{D}}$ of point $\bar{P}$ at instant $t>0$. in a meridian plane, the $\bar{\xi} \sigma$ plane, with origin $\bar{P}$. With length scaled by $C t$, the domain $\overline{\mathcal{D}}$ is the unit semicircular disk above the $\bar{\xi}$ axis. A fixed point $\bar{S}$ shown at $t=1$ is moving towards the origin as $t$ increases. Also shown is the partition of $\overline{\mathcal{D}}$ into two nonoverlapping regions, $\bar{V}^{ \pm}$ by the radial line $\bar{P} \bar{T}$ at $\psi=\arccos 1 / M$ representing the critical curve for a constant supersonic speed at Mach number $M=\sqrt{5}$. 


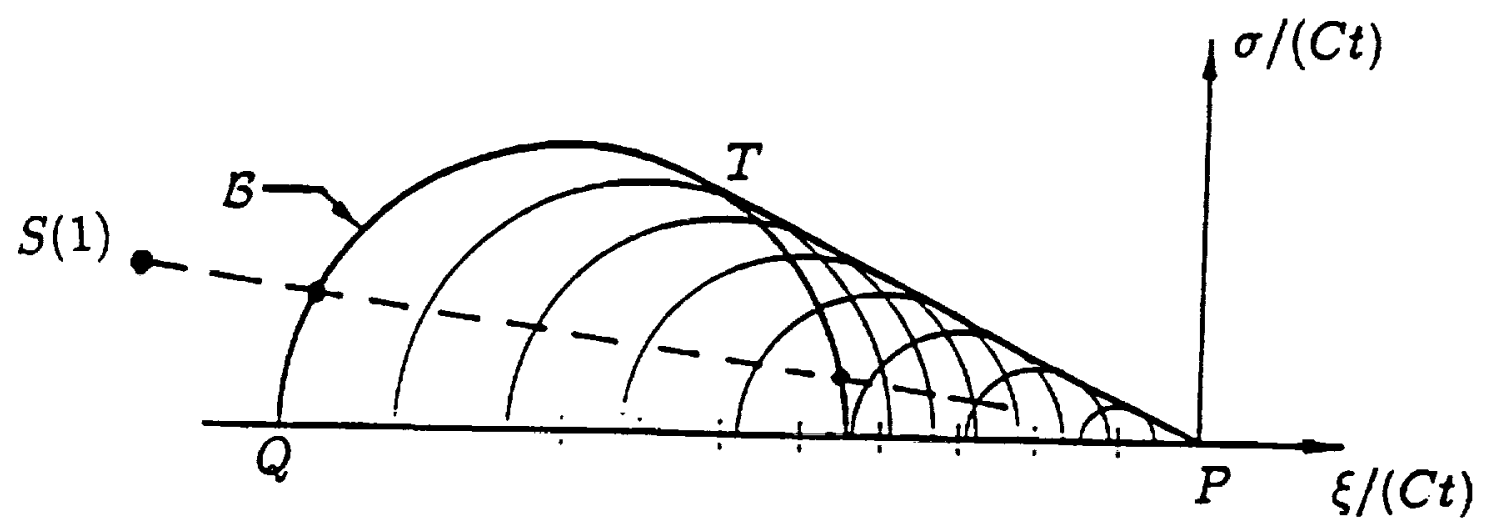

Fig. 3 The domain $\mathcal{D}$ and point $P$ are the images of $\overline{\mathcal{D}}$ and $\bar{P}$ in Fig. 2. The domain $\mathcal{D}$, which depends on the relative velocity $U \hat{\imath}$, is shown here in the meridian $\xi \sigma$ plane with origin $P$ for a constant supersonic speed at $M=\sqrt{5}$, with length scaled by $C t$.

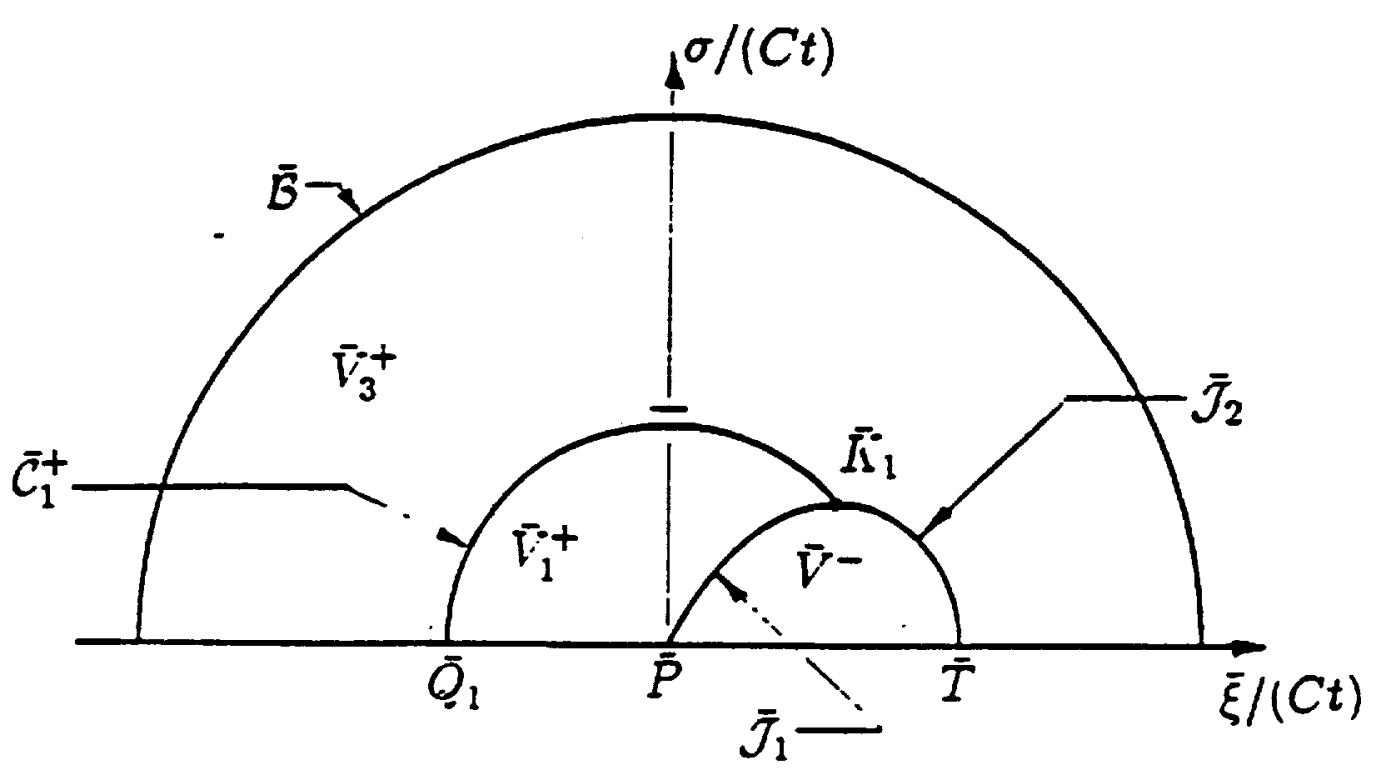

Fig. 4 The partition of the domain of dependence $\overline{\mathcal{D}}$ of point $\bar{P}$ in a meridian $\bar{\xi} \sigma$ plane for a constantly accelerating motion. Here $U(\tau)=C \tau, \tau \in[0, \sqrt{5}]$, with final Mach number $M=\sqrt{5}$. The critical curve $\overline{\mathcal{J}}$ has a maximum $\sigma\left(\tau_{1}\right)$ at point $\bar{K}_{1}$ with $\tau_{1}=5^{1 / 6}$. The point $\bar{K}_{1}$ divides $\overline{\mathcal{J}}$ into $\overline{\mathcal{J}}_{1}$ and $\overline{\mathcal{J}}_{2}$. The critical curve partitions $\overline{\mathcal{D}}$ into $\bar{V}^{ \pm}$. The circular arc $\overline{\mathcal{C}}_{1}^{+}$partitions $V^{+}$into $V_{1}^{+}$and $\bar{V}_{3}^{+}$. 

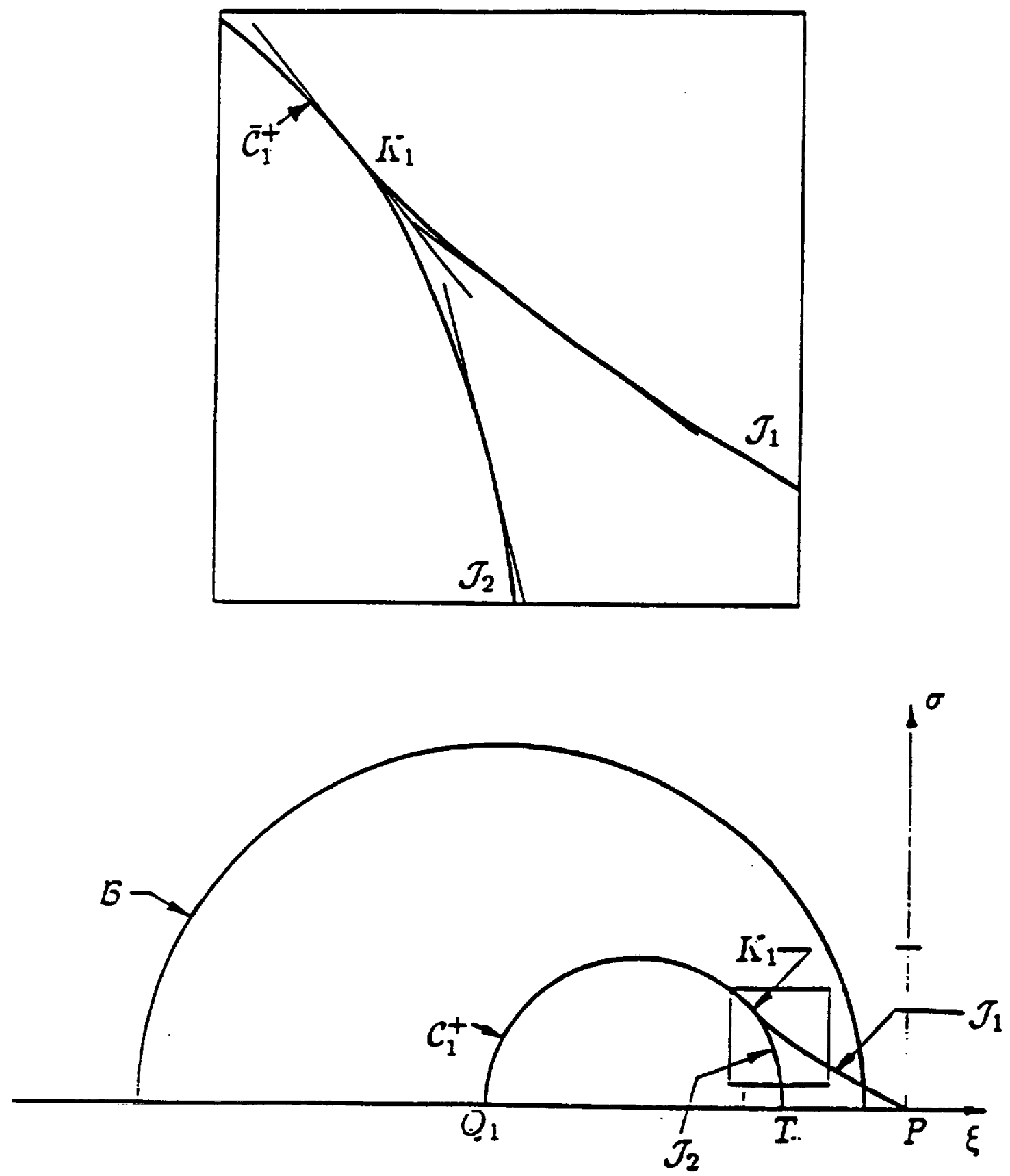

Fig. 5 Images of curves shown in Fig. 4 in the $\xi \sigma$ plane. They are the semicircle $\mathcal{B}$, two branches of the critical curve $\mathcal{J}, \mathcal{J}_{1}$ and $\mathcal{J}_{2}$, and the circular arc $\mathcal{C}_{1}^{+}$through the cusp $K_{1}$ on $\mathcal{J}$. An enlargement of the neighborhood of the cusp $K_{1}$ is shown in the insert. Also shown are the common tangent to the two branches, and the tangents at points on the branches. These tangent lines are the local Mach lines. 


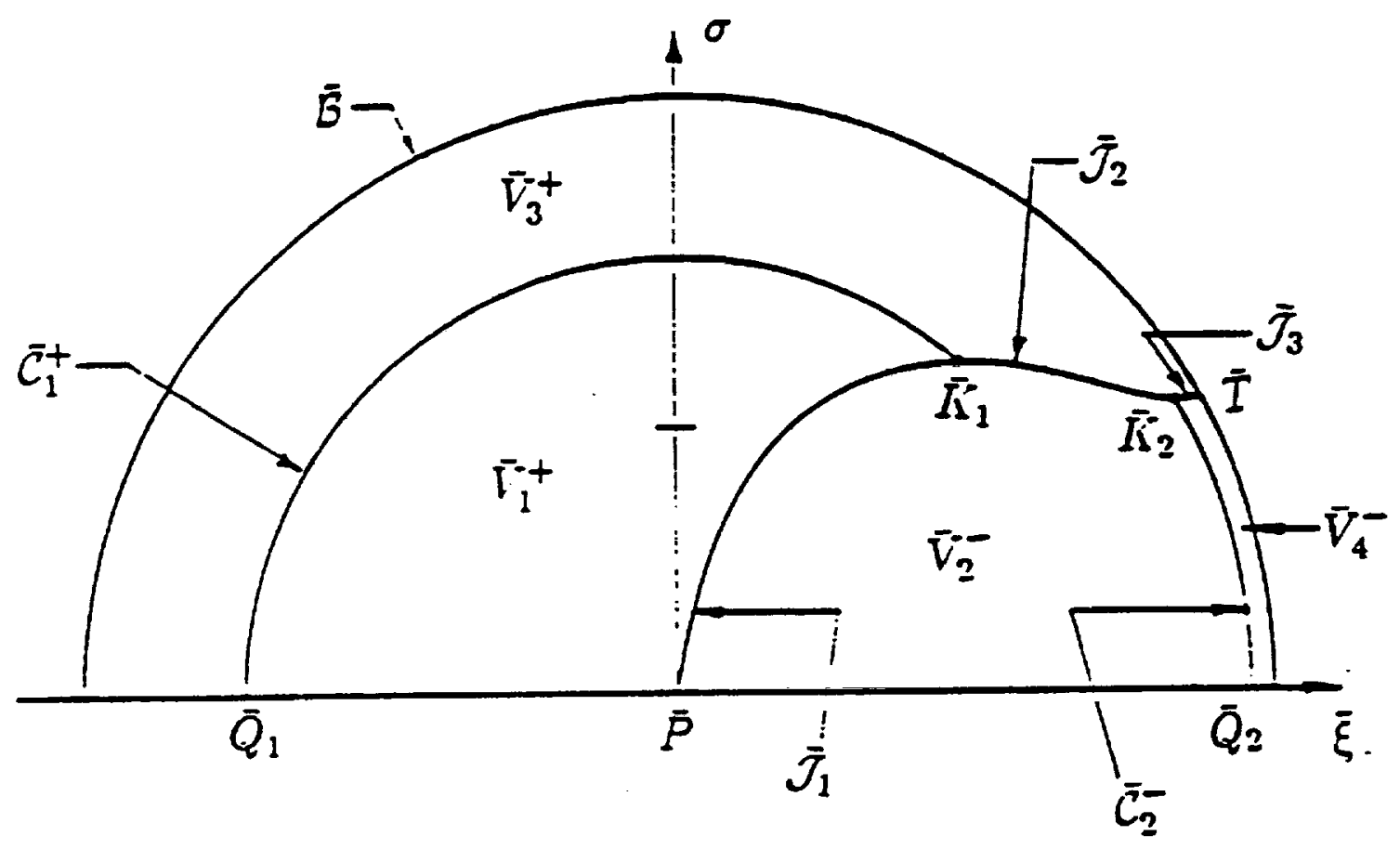

Fig. 6 The partition of the domain of dependence $\overline{\mathcal{D}}$ of point $\bar{P}$ in a meridian $\bar{\xi} \sigma$ plane for a constant rate acceleration from a low supersonic speed. Here $M(\tau)=1.15+\tau^{2}, \tau \in$ $[0,2.25]$, with final Mach number $M=6.2125$. The critical curve $\overline{\mathcal{J}}$ has a local maximum at point $\bar{K}_{1}$ and minimum at point $\bar{K}_{2}$. The curve partitions $\overline{\mathcal{D}}$ into $\bar{V}^{ \pm}$. $\bar{V}^{+}$is partitioned by the circular arc $\overline{\mathcal{C}}_{1}^{+}$starting from point $\bar{K}_{1}$ into $\bar{V}_{1}^{+}$and $\bar{V}_{3}^{+}$, and $\bar{V}^{-}$by $\operatorname{arc} \overline{\mathcal{C}}_{2}^{-}$from point $\bar{K}_{2}$ into $\bar{V}_{2}^{-}$and $\bar{V}_{4}^{-}$. 

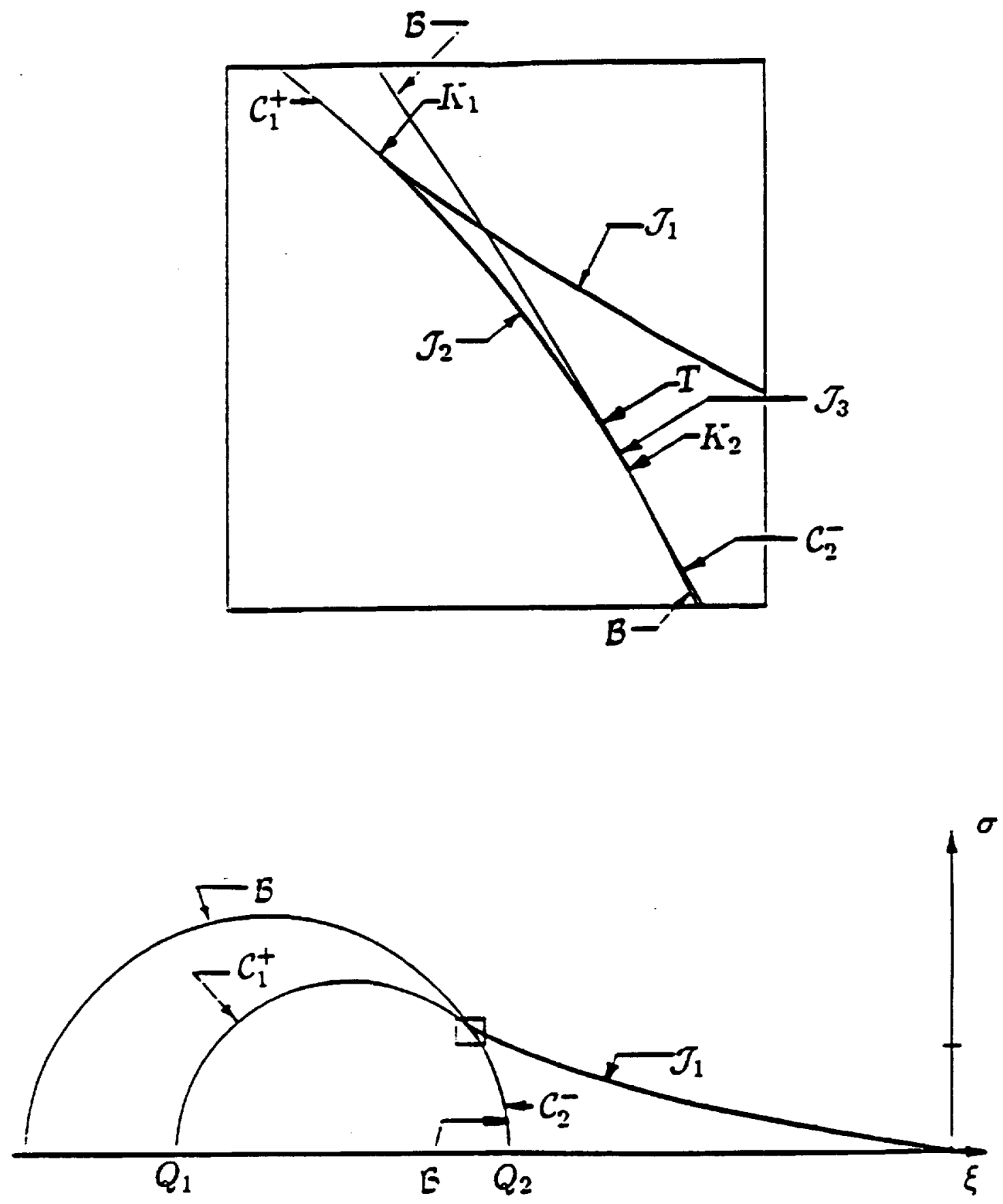

Fig. 7 Images of curves shown in Fig. 6 in the $\xi \sigma$ plane. They are the smirirrle $\mathcal{B}$, the three branches of the critical curve, $\mathcal{J}_{1}, \mathcal{J}_{2}$ and $\mathcal{J}_{3}$, and two circular arc $\mathcal{C}_{1}$ and $\mathcal{C}_{2}^{-}$ from the two cusps $K_{1}$ and $K_{2}$ on $\mathcal{J}$. Note the small difference between $\mathcal{C}_{2}^{-}$, and the semicircle $\mathcal{B}$. A $20 \times$ enlargement of the neighborhood of the cusps $K_{2}$ and $K_{3}$ is shown in the insert. Note that the branch $\mathcal{J}_{3}$ begins at point $T$ tangent to the semicircle $\mathcal{B}$ and continues downward to the right hand side of $\mathcal{B}$. 

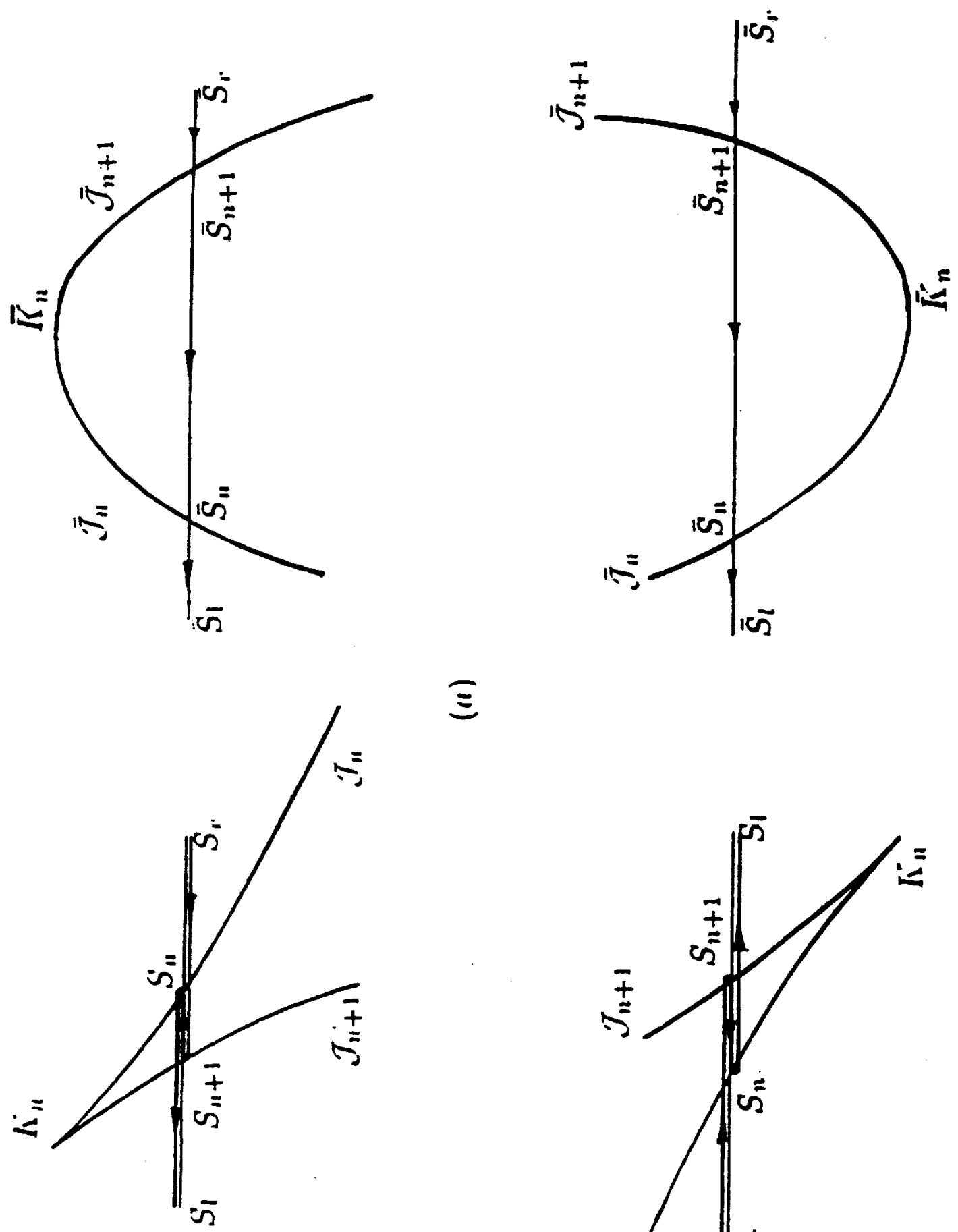

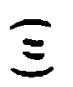

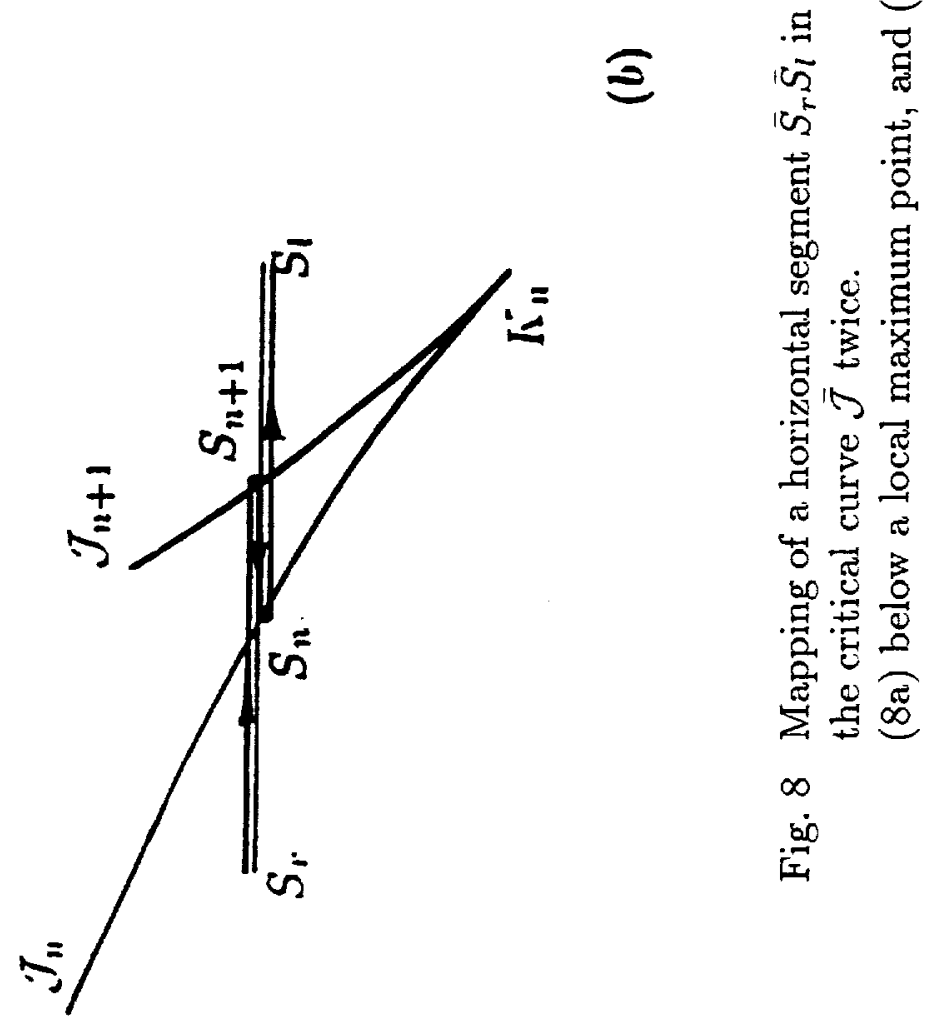




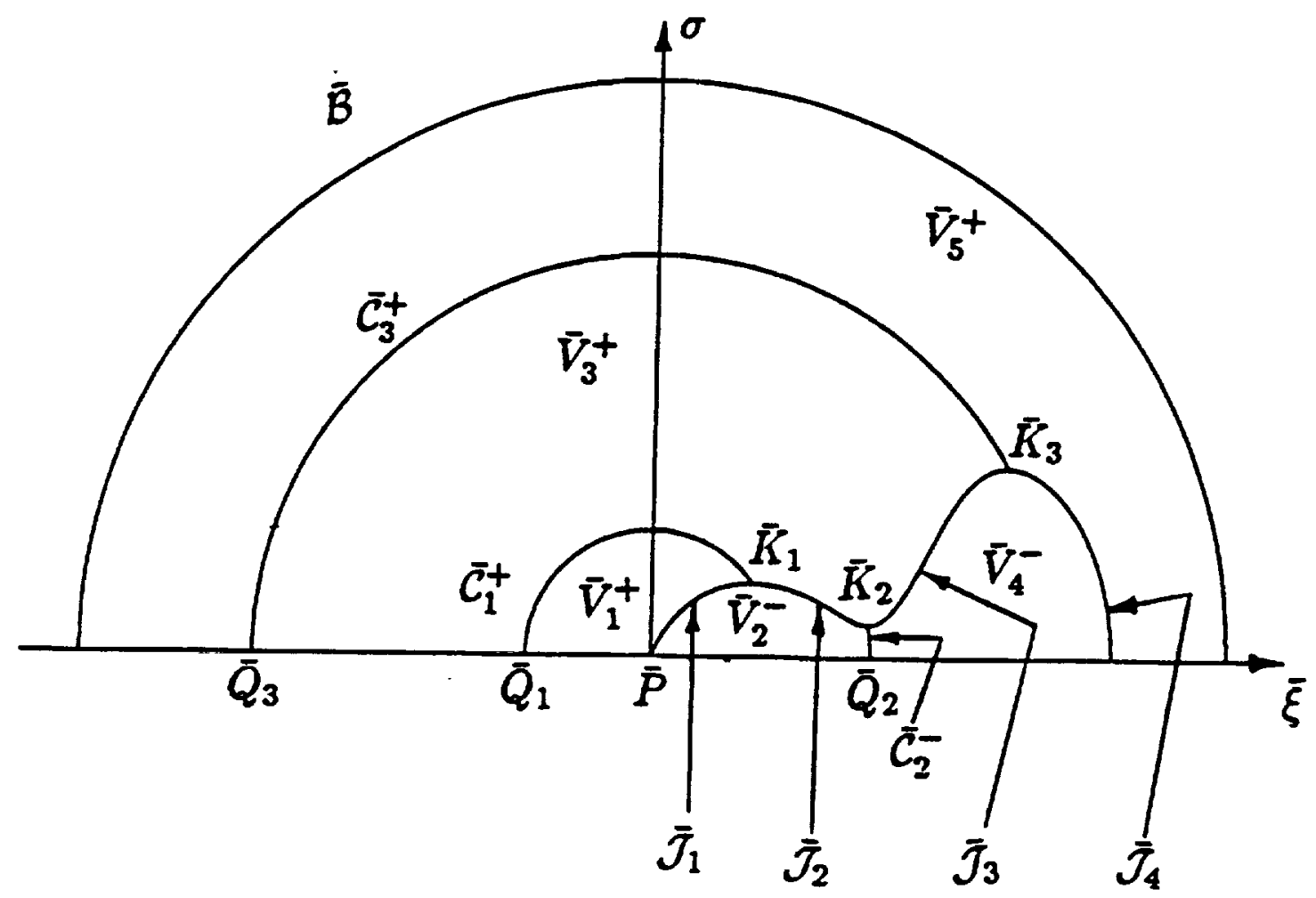

Fig. 9 The partition of the domain of dependence $\overline{\mathcal{D}}$ of point $\bar{P}$ in a meridian $\bar{\xi} \sigma$ plane for an accelerating, decelerating and accelerating supersonic speed starting from rest at $\tau=0$. The subsonic and supersonic intervals are $[0,1)$ and $(1,5]$ with the deceleration in $(1.7,3.05)$. The critical curve $\overline{\mathcal{J}}$ has a local maximum at point $\bar{K}_{1}$, a minimum at $\bar{K}_{2}$ and then a maximum at $\bar{K}_{3}$. The curve $\overline{\mathcal{J}}$ partitions $\overline{\mathcal{D}}$ into $\bar{V}^{ \pm} . \bar{V}^{-}$is partitioned by the circular arc $\overline{\mathcal{C}}_{2}^{-}$starting from point $\bar{K}_{2}$ into two subdomains $\bar{V}_{2}^{-}$and $\bar{V}_{4}^{-}$, and $\bar{V}^{+}$by $\operatorname{arcs} \overline{\mathcal{C}}_{1}^{+}$from point $\bar{K}_{1}$ and $\overline{\mathcal{C}}_{3}^{+}$from point $\bar{K}_{3}$ into three domains $\bar{V}_{1}^{+}, \bar{V}_{3}^{+}$and $\bar{V}_{5}^{+}$. 

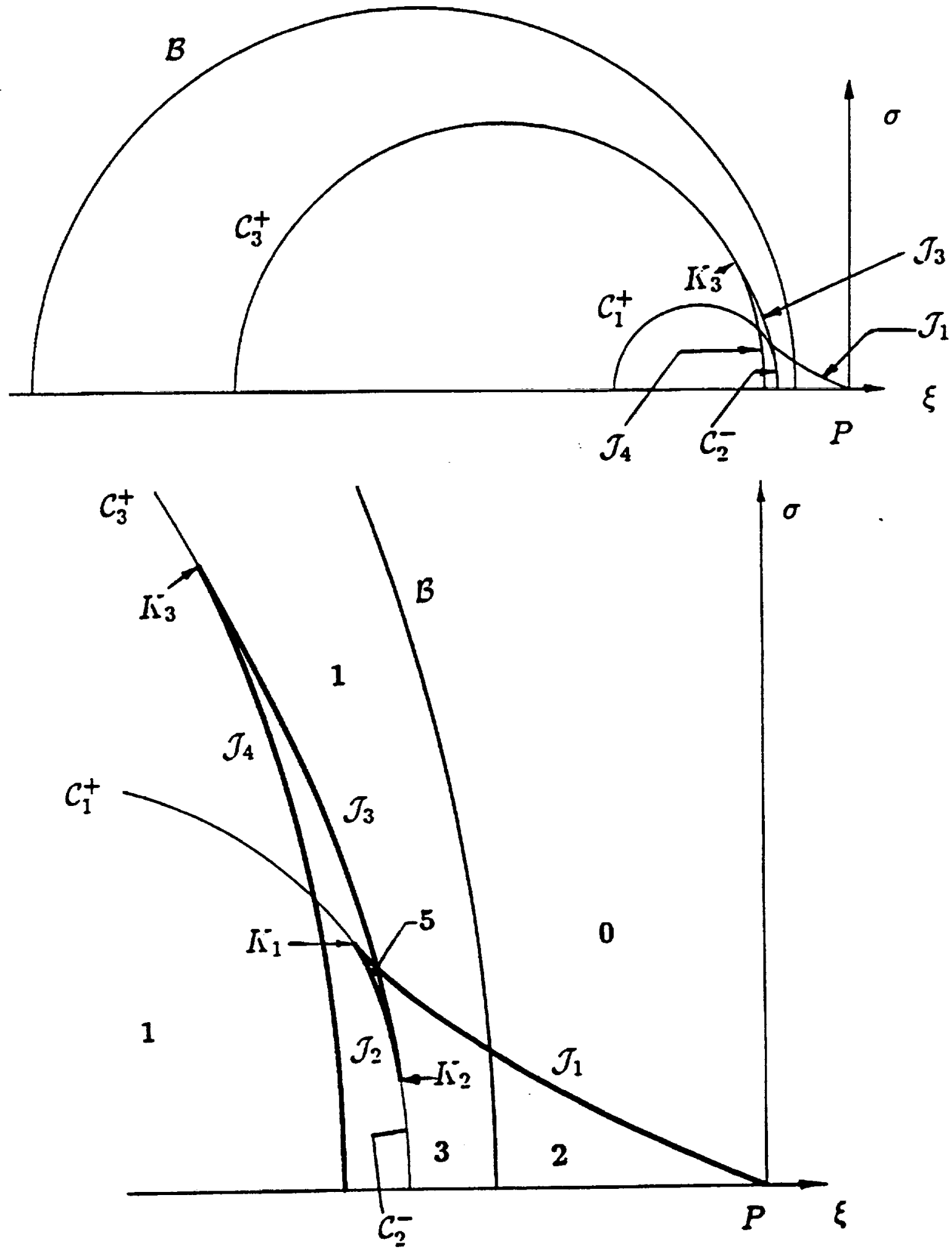

Fig. 10 Images of the five nonoverlapping subdomains of $\overline{\mathcal{D}}$, shown in Fig. 9. ar" superimposed onto the $\xi \sigma$ plane in (10a). Shown in Fig. (10b) is the $5 \times$ enlargement of the region containing the four branches of the critical curve, $\mathcal{J}_{i}, i=1,2,3,4$. The numerals in bold face indicate the multiplicities of the regions bounded by $\mathcal{J}_{i}, \mathcal{B}$ and/or the $\xi$-axis. 


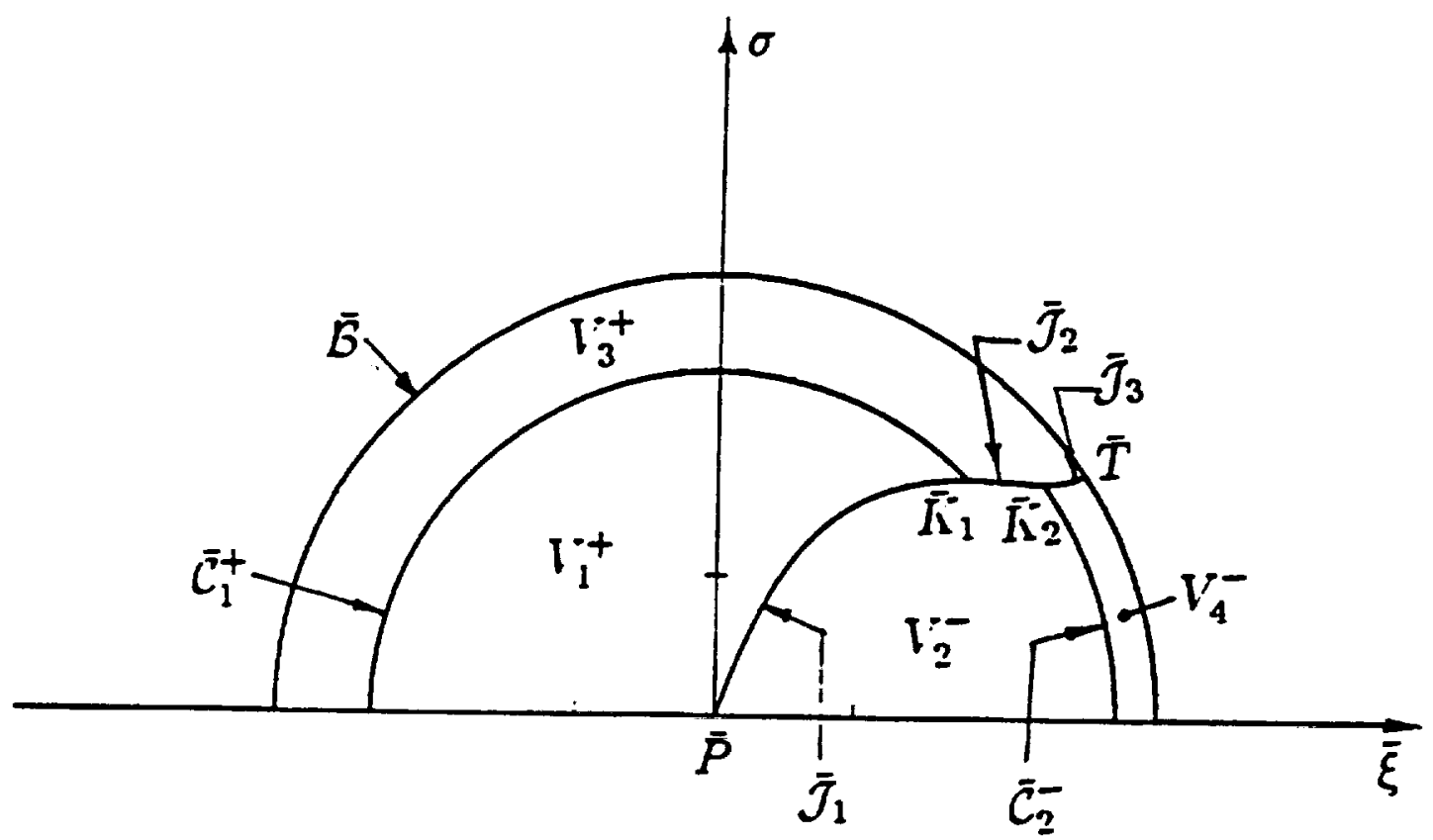

Fig. 11 The partition of the domain of dependence $\overline{\mathcal{D}}$ of point $\bar{P}$ at time $t$ for a supersonic periodic motion $M(\tau)=2-0.8 \cos \tau$ for $0 \leq \tau \leq t=\pi$ with $M^{\prime}(\tau) \geq 0$. 

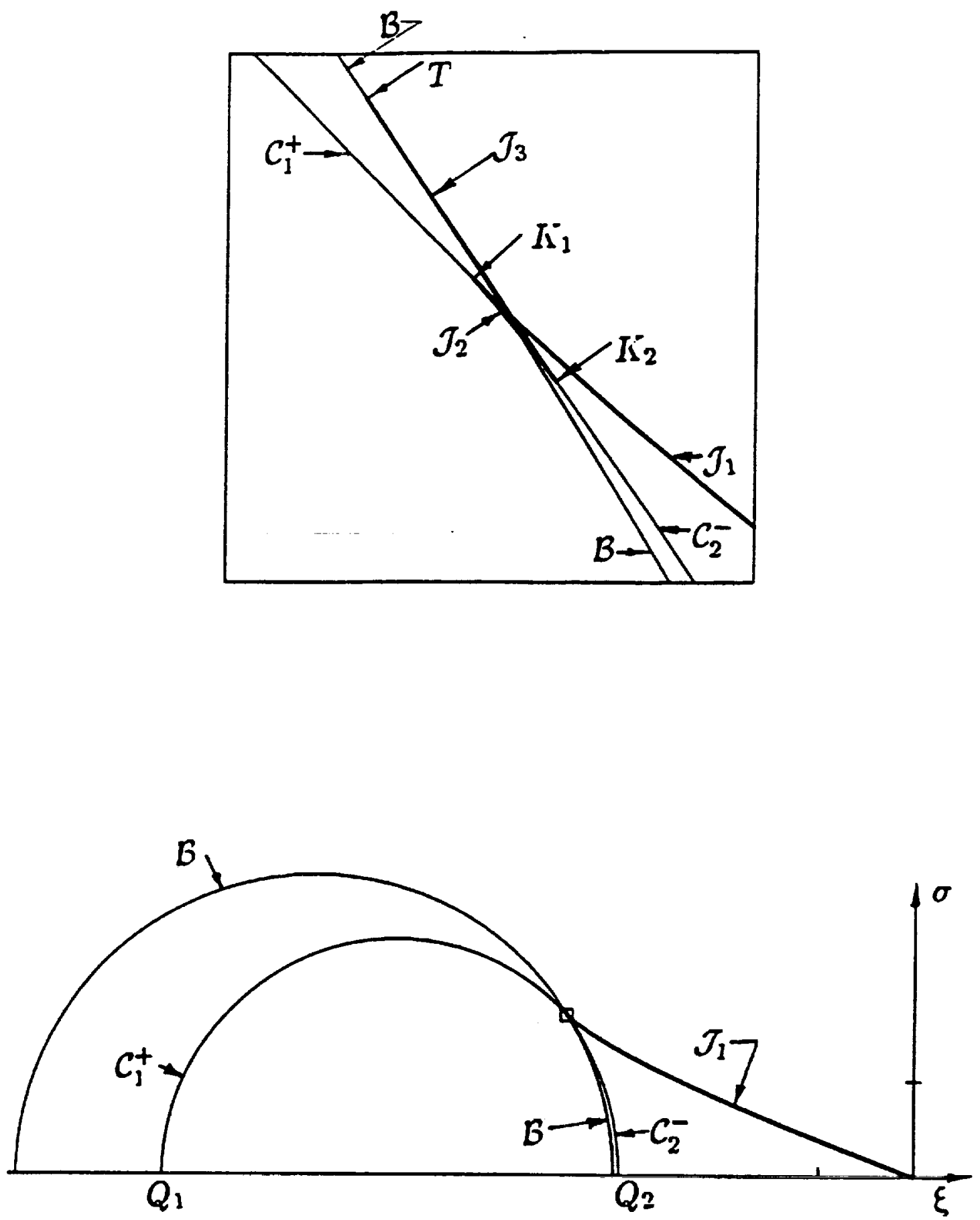

Fig. 12 Images of the four nonoverlapping subdomains of $\overline{\mathcal{D}}$ shown in Fig. 11 are superposed on to the $\xi \sigma$ plane in Fig. 12a. Shown in Fig. 12b is the $45 \times$ enlargement of the region containing the two cusps. 


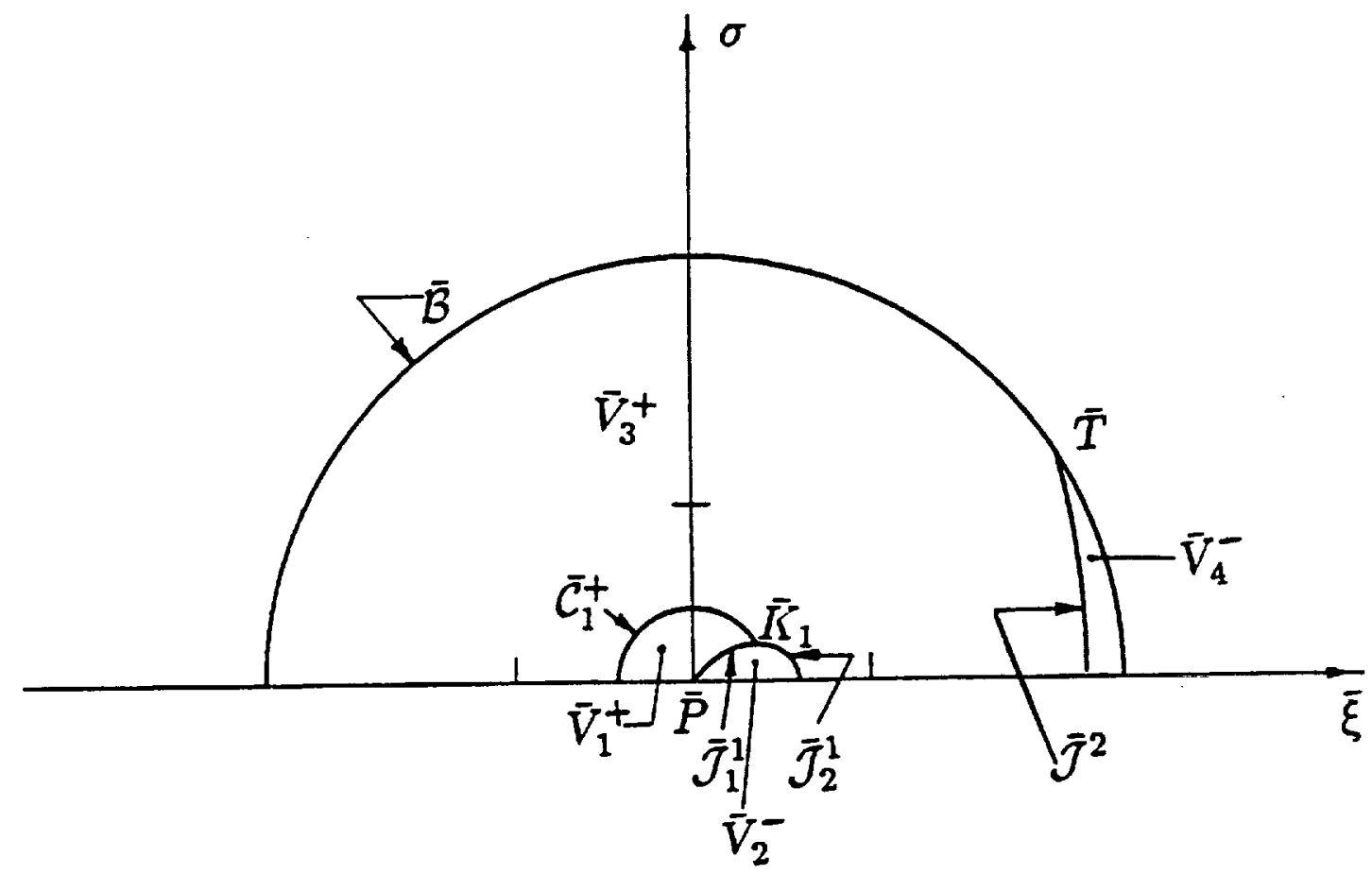

Fig. 13 The partition of the domain of dependence $\overline{\mathcal{D}}$ of point $\bar{P}$ at time $t$ with two supersonic intervals, $M(\tau)=M_{0}-b \tau+\tau^{2} / 2$ for $0 \leq \tau \leq t$, with $M_{0}=1.18, b=1.0$ and $t=2.4$. 

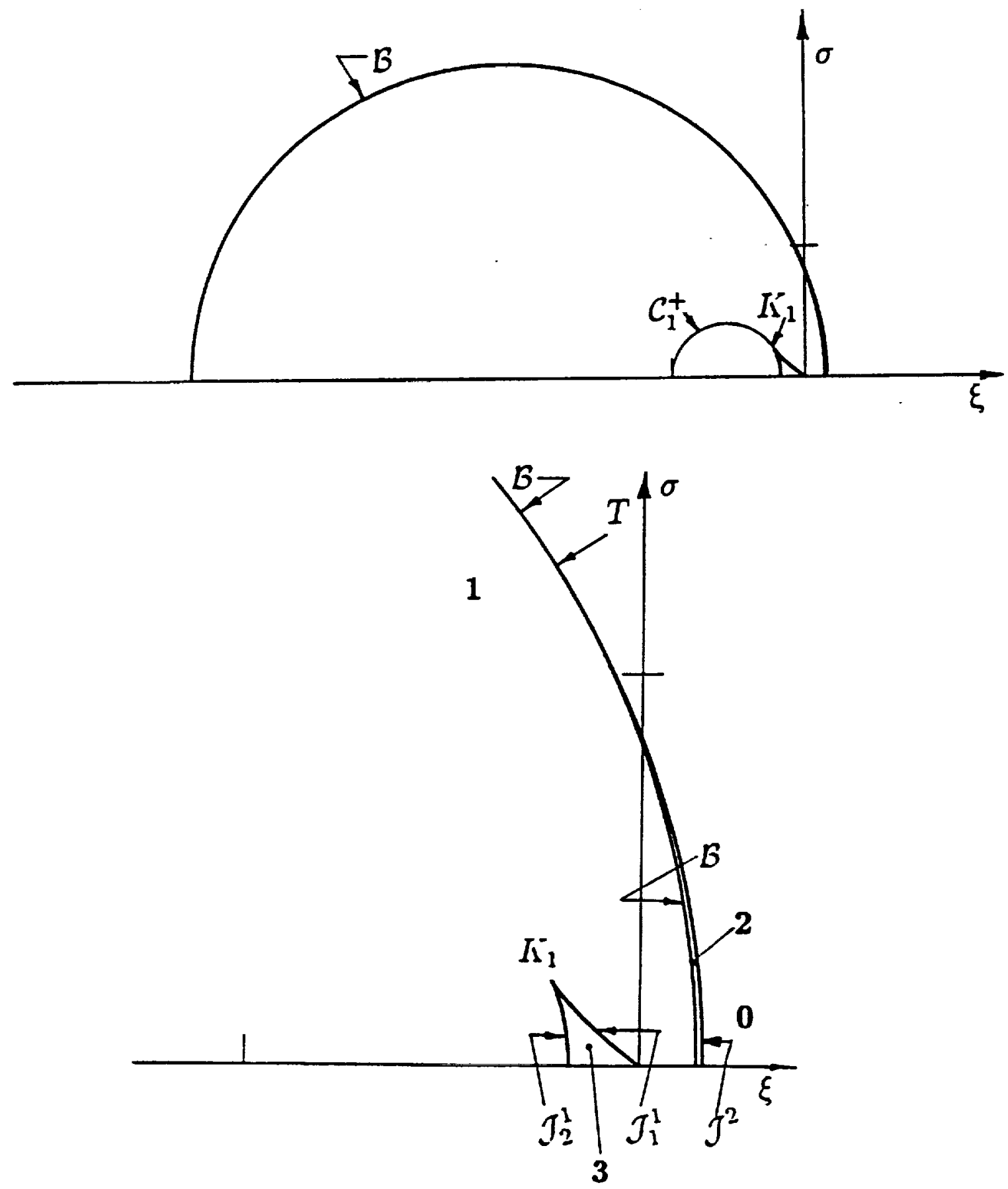

Fig. 14 Images of the four nonoverlapping subdomains of $\overline{\mathcal{D}}$ shown in Fig. 13 are superposed on to the $\xi \sigma$ plane in Fig. 14a. Shown in Fig. $14 \mathrm{~b}$ is the $3 \times$ enlargement of the region containing two nonintersecting critical curves. 


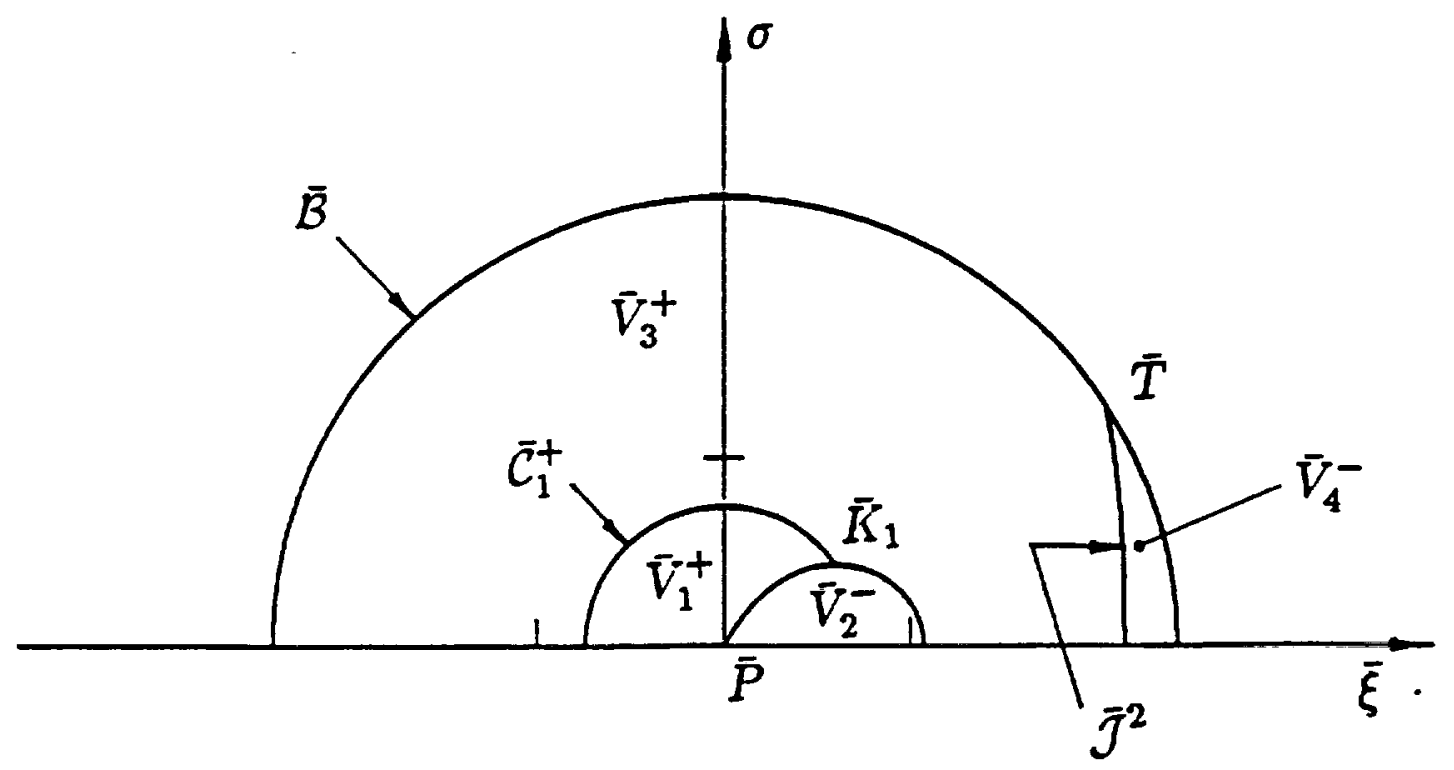

Fig. 15 The partition of the domain of dependence $\overline{\mathcal{D}}$ of point $\bar{P}$ at time $t$ with two supersonic intervals, $M(\tau)=M_{0}-b \tau+\tau^{2} / 2$ for $0 \leq \tau \leq t$, with $M_{0}$ and $t$ the same as in Fig. 13 but $b=0.8$. 

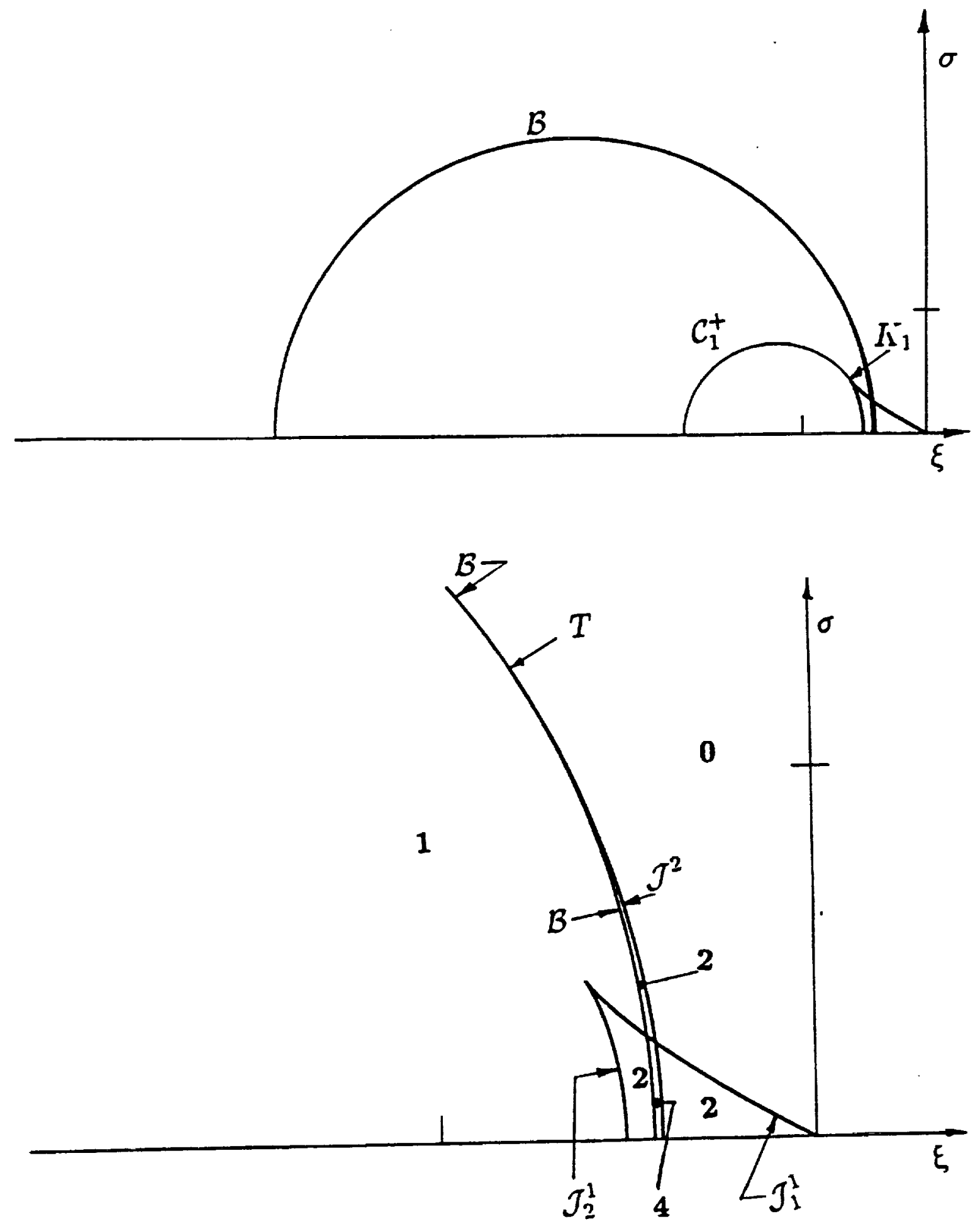

Fig. 16 Images of the four nonoverlapping subdomains of $\overline{\mathcal{D}}$ shown in Fig. 15 are superposed on to the $\xi \sigma$ plane in Fig. 16a. Shown in Fig. $16 \mathrm{~b}$ is the $3 \times$ enlargement of the region containing two intersecting critical curves . 


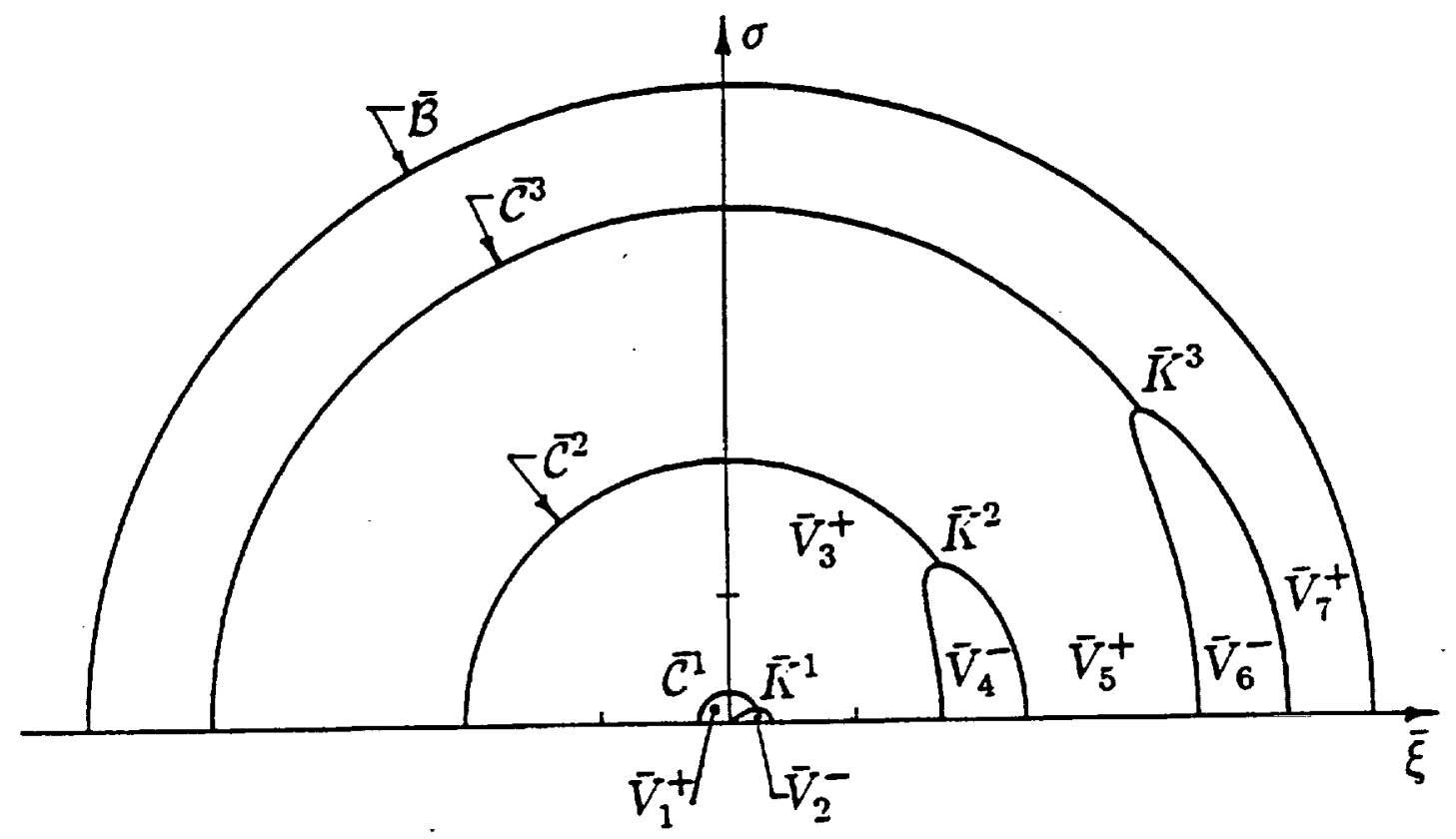

Fig. 17 The partition of the domain of dependence $\overline{\mathcal{D}}$ of point $\bar{P}$ at time $t$ with three supersonic intervals, $M(\tau)=0.75-0.5 \cos (\pi \tau)$ for $0 \leq \tau \leq t=5$. 

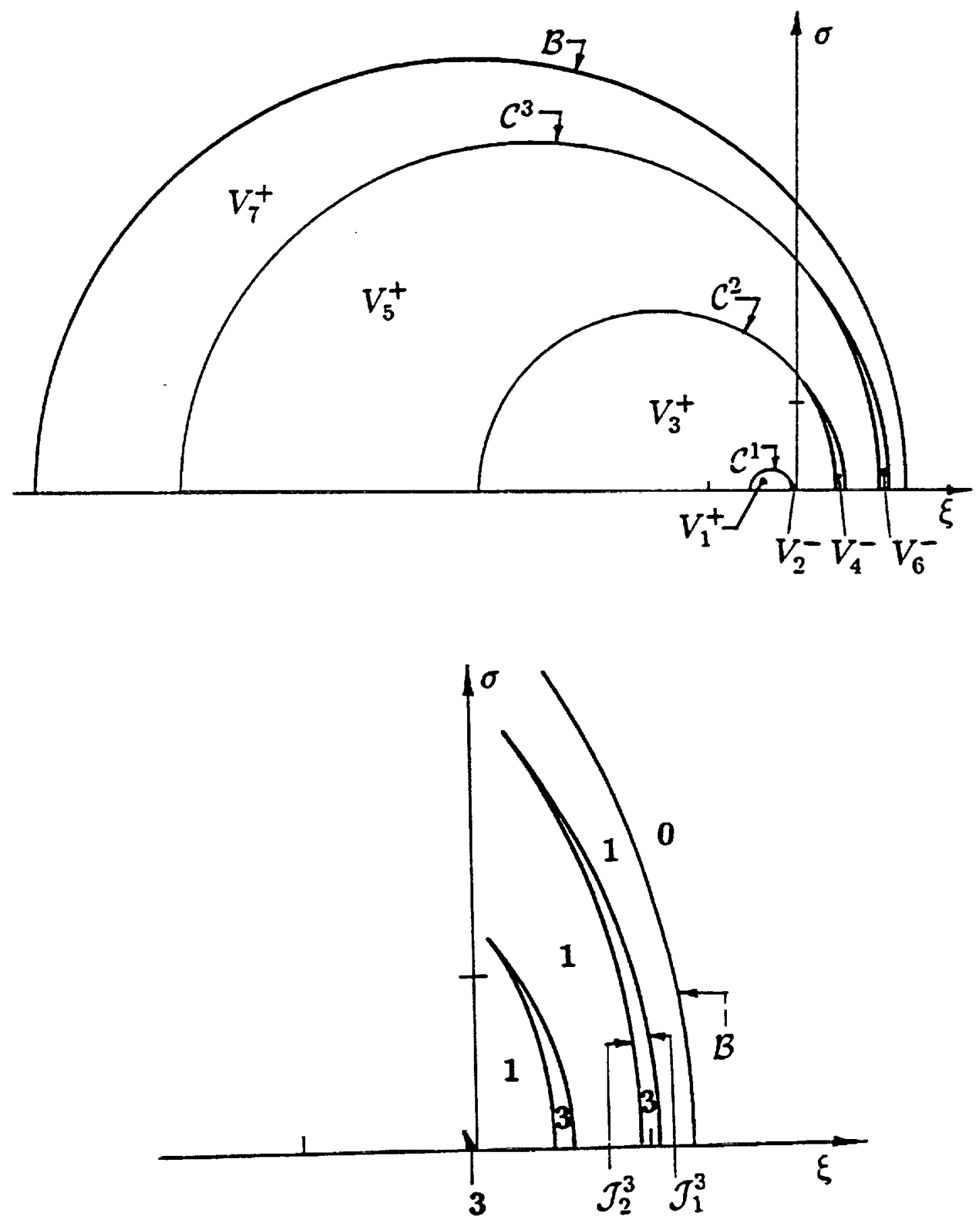

Fig. 18 Images of the seven nonoverlapping subdomains of $\overline{\mathcal{D}}$ shown in Fig. 17 are superposed on to the $\xi \sigma$ plane in Fig. 18a. Shown in Fig. $18 \mathrm{~b}$ is the $2 \times$ enlargement of the region containing two nonintersecting critical curves. 

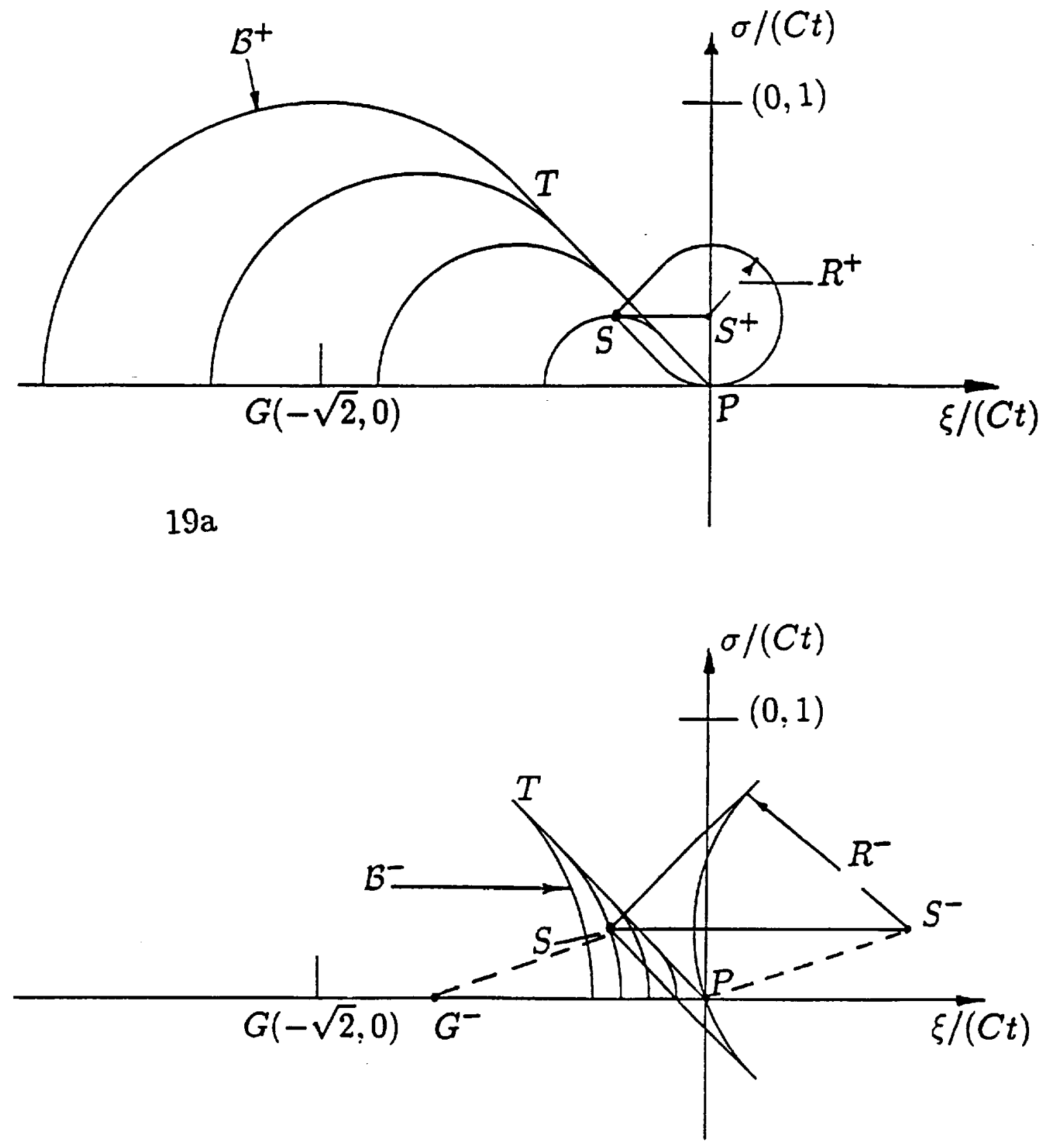

$19 b$

Fig. 19 Propagation of signals from point $S$ initiated at different retarded times to the observation point $P$ at time $t$, as explained in Appendix A. 


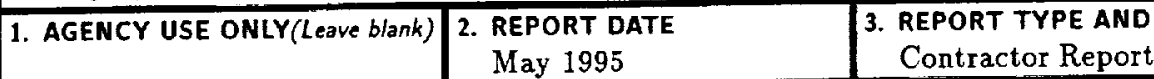

\begin{tabular}{ll|l} 
4. TITLE AND SUBTITLE & 5. FUNDING NUMBERS
\end{tabular}

ACOUSTIC FIELD IN UNSTEADY MOVING MEDIA $\mid$ C NAS1-19480

6. AUTHOR(S)

F. Bauer

L. Maestrello

L. Ting

7. PERFORMING ORGANIZATION NAME(5) AND ADDRESS(ES)

Institute for Computer Applications in Science

and Engineering

Mail Stop 132C, NASA Langley Research Center

WU 505-90-52-01

Hampton, VA 23681-0001

9. SPONSORING/MONITORING AGENCY NAME(S) AND ADDRESS(ES)

National Aeronautics and Space Administration

Langley Research Center

Hampton, VA 23681-0001

8. PERFORMING ORGANIZATION REPORT NUMBER

ICASE Report No. 95-39

11. SUPPLEMENTARY NOTES

Langley Technical Monitor: Dennis M. Bushnell

Final Report

Submitted to JASA

12a. DISTRIBUTION/AVAILABILITY STATEMENT

12b. DISTRIBUTION CODE

Unclassified-Unlimited

Subject Category 34

13. ABSTRACT (Maximum 200 words)

In the interaction of an acoustic field with a moving airframe we encounter a canonical initial value problem for an acoustic field induced by an unsteady source distribution, $q(t, \mathbf{x})$ with $q \equiv 0$ for $t \leq 0$, in a medium moving with a uniform unsteady velocity $U(t) \hat{\imath}$ in the coordinate system $x$ fixed on the airframe. Signals issued from a source point $S$ in the domain of dependence $D$ of an observation point $P$ at time $t$ will arrive at point $P$ more than once corresponding to different retarded times, $\tau$ in the interval $[0, t]$. The number of arrivals is called the multiplicity of the point $S$. The multiplicity equals 1 if the velocity $U$ remains subsonic and can be greater when $U$ becomes supersonic. For an unsteady uniform flow $U(t) \hat{i}$, rules are formulated for defining the smallest number of $I$ subdomains $V_{i}$ of $\mathcal{D}$ with the union of $V_{i}$ equal to $\mathcal{D}$. Each subdomain has multiplicity 1 and a formula for the corresponding retarded time. The number of subdomains $V_{i}$ with nonempty intersection is the multiplicity $m$ of the intersection. The multiplicity is at most $I$. Examples demonstrating these rules are presented for media at accelerating and/or decelerating supersonic speed.

14. SUBJECT TERMS
Unsteady linear acoustics;
17. SECURITY CLASSIFICATION
OF REPORT
Unclassified

NSN 7540-01-280-5500

\begin{tabular}{|l|l|} 
& $\begin{array}{c}\text { 15. NUMBER OF PAGES } \\
47\end{array}$ \\
\cline { 2 - 3 } & $\begin{array}{c}\text { 16. PRICE CODE } \\
\text { AO3 }\end{array}$ \\
\hline $\begin{array}{l}\text { 19. SECURITY CLASSIFICATION } \\
\text { OF ABSTRACT }\end{array}$ & $\begin{array}{l}\text { 20. LIMITATION } \\
\text { OF ABSTRACT }\end{array}$ \\
\hline $\begin{array}{l}\text { Standard Form 298(Rey. 2-89) } \\
\text { Prescribed by ANSI Sid. Z39-18 } \\
\text { 298-102 }\end{array}$
\end{tabular}

18. SECURITY CLASSIFICATION OF THIS PAGE Unclassified
SPONSORING/MONITORING

NASA CR-198162

ICASE Report No. 95-39 

. 
